\title{
The Multi-Objective Design of Flatback Wind Turbine Airfoils
}

\author{
by \\ Michael Miller, B.Eng. \\ A thesis submitted to the \\ Faculty of Graduate and Postdoctoral Affairs \\ in partial fulfillment of the requirements for the degree of
}

Master of Applied Science in Mechanical Engineering

Ottawa-Carleton Institute for Mechanical and Aerospace Engineering

Department of Mechanical and Aerospace Engineering

Carleton University

Ottawa, Ontario

December, 2016

(C) Copyright

Michael Miller, 2016 
The undersigned hereby recommends to the

Faculty of Graduate and Postdoctoral Affairs acceptance of the thesis

\title{
The Multi-Objective Design of Flatback Wind Turbine Airfoils
}

\author{
submitted by Michael Miller, B.Eng. \\ in partial fulfillment of the requirements for the degree of \\ Master of Applied Science in Mechanical Engineering
}

Professor Edgar Matida, Thesis Supervisor

Professor Ron Miller, Chair, Department of Mechanical and Aerospace Engineering

Ottawa-Carleton Institute for Mechanical and Aerospace Engineering Department of Mechanical and Aerospace Engineering Carleton University

December, 2016 


\section{Abstract}

With the ever increasing lengths of today's wind turbine rotor blades, there is a need for airfoils which are both aerodynamically, and structurally efficient. In this work, a multi-objective genetic algorithm coupled with XFOIL was developed to design flatback wind turbine airfoils. The effect of the aerodynamic evaluator, specifically lift-to-drag ratio, torque, and torque-to-thrust ratio, on the airfoil shape and performance was examined. Under the specified set of constraints and objectives, notable differences, particularly in the levels of lift and roughness insensitivity, were observed. Further analysis, employing the Taguchi method, was performed to determine how various parameters impact the design outcome. The obtained knowledge was used to design a wind turbine specific airfoil family which has comparable, or superior structural and aerodynamic performance as compared to airfoils found in the literature.

A wind tunnel experimental set-up was developed at the Carleton University Low Speed Wind Tunnel for the 2D testing of airfoils. Good agreement between the results obtained at Carleton University and other publicly available data indicates that the set-up is capable of producing meaningful data. A select airfoil, designed by the current author, was tested at Carleton University and its performance is compared to the numerical predictions of XFOIL. Differences in the stall and post-stall regions of the XFOIL predictions are highlighted, and emphasize the importance of wind tunnel testing in the airfoil design process. 


\section{Acknowledgments}

I would like to first thank my thesis supervisor, Dr. Edgar Matida, for the opportunity to pursue this degree. His guidance, advice, and support made these past few years more enjoyable and a constant learning experience for me, both academically and practically. Most importantly, his trust encouraged me to achieve more than I thought was possible, and kept me motivated throughout.

I would also would like to give thanks to Alex, Kevin and Ian from the machine shop for letting me barge in countless times, and assisting me in the manufacturing of the equipment necessary for my work. I must also acknowledge Dr. Ahmadi for lending me equipment that I required for my experiments. Next, I'd like to thank all of my labmates who provided me with many essential lunch, coffee, and bagel distractions which kept me sane and well-fed. A special mention has to go to my friend and colleague Kenny who shared a large portion of these past two years working alongside me; his assistance in every aspect of my work is greatly appreciated.

I would like to thank all of my friends who were always willing to provide me with social distractions and gave me balance in life. Next, I want to thank my girlfriend Bronwen who showed patience, understanding, and support no matter what the situation, and truly made these past years more manageable. Finally, I would like to thank my family. Without their support, words of praise, and encouragement, I do not think I would be where I am today. 


\section{Table of Contents}

Abstract $\quad$ iii

Acknowledgments $\quad$ iv

Table of Contents $\quad$ v

List of Tables viii

List of Figures $\quad$ ix

Nomenclature $\quad$ xiv

1 Introduction and Motivation 1

1.1 Current State of Horizontal-Axis Wind Turbines . . . . . . . . . . . . 3

1.2 Aerodynamics of Horizontal-Axis Wind Turbines . . . . . . . . . . . . 5

1.3 Motivation ......................... 7

1.4 Outline . . . . . . . . . . . . . . . . . . . . 9

2 Literature Review and Background 10

2.1 Aerodynamics of Airfoils . . . . . . . . . . . . . . . . . . 10

2.2 Flatback Airfoils . . . . . . . . . . . . . . . . . 24

2.3 Design of Wind Turbine Airfoils . . . . . . . . . . . . . . . . . 32 
3.1 Fundamentals of Numerical Optimization . . . . . . . . . . . 40

3.1 .1 Terminology . . . . . . . . . . . . . . . . . . . . 41

3.1 .2 Optimization Techniques . . . . . . . . . . . . . . . . 42

3.2 Implementation of Optimization for Flatback Airfoils . . . . . . . . 44

3.2 .1 MATLAB gamultiobj . . . . . . . . . . . . . . . 45

3.2 .2 Airfoil Shape Generator _. . . . . . . . . . . . . . . . 51

3.2 .3 Coupler . . . . . . . . . . . . . . . . . . 56

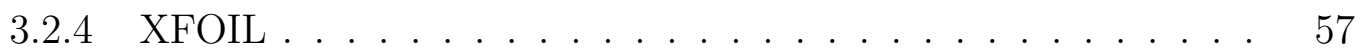

3.3 Convergence Analysis . . . . . . . . . . . . . . . . . . . . 62

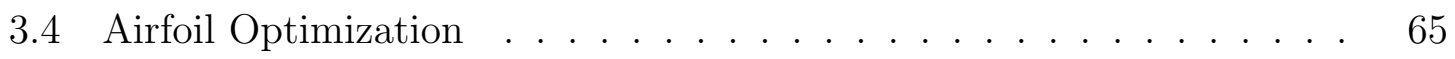

3.4.1 Design and Optimization Parameters . . . . . . . . . 65

3.4.2 Aerodynamic Evaluator Results . . . . . . . . . . . . . 68

3.4.3 Summary of Aerodynamic Evaluator Findings . . . . . . . 78

3.5 Constraint and Objective Sensitivity . . . . . . . . . . 79

3.6 Airfoil Family . . . . . . . . . . . . . . . . . . 86

4 Experimental Characterization of Airfoils $\quad 93$

4.1 Equipment and Procedure . . . . . . . . . . . . . . . . . 94

4.1.1 Wind Tunnel Facility and Equipment . . . . . . . . . . 95

4.1 .2 Airfoil Model . . . . . . . . . . . . . . . . . . . . . . 99

4.1 .3 Experimental Procedure . . . . . . . . . . . . . . . . . . . 101

4.1 .4 Data Reduction . . . . . . . . . . . . . . . . . . . 103

4.2 Wind Tunnel Corrections . . . . . . . . . . . . . . . . . 105

4.2 .1 Solid Blockage . . . . . . . . . . . . . . . . . . 105

4.2 .2 Wake Blockage . . . . . . . . . . . . . . . . 106 
4.2 .3 Streamline Curvature . . . . . . . . . . . . . . . . 106

4.2.4 Summary of Corrections . . . . . . . . . . . . . . . . 107

4.3 Results. . . . . . . . . . . . . . . . . . . . 108

4.3.1 S809 Airfoil Validation . . . . . . . . . . . . . . . 111

4.3.2 $\mathbf{C}_{\mathbf{T}}$ Airfoil Performance . . . . . . . . . . . . . 115

4.3.3 Uncertainty Analysis . . . . . . . . . . . . . . . . . 121

5 Conclusions and Future Work 130

5.1 Numerical Work . . . . . . . . . . . . . . . . . 130

5.1 .1 Conclusions . . . . . . . . . . . . . . . . 130

5.1 .2 Future Work . . . . . . . . . . . . . . . . . . 132

5.2 Experimental Work . . . . . . . . . . . . . . . 134

5.2.1 Conclusions . . . . . . . . . . . . . . . . . . . . . 134

5.2 .2 Future Work . . . . . . . . . . . . . . . 136

5.3 Concluding Remarks . . . . . . . . . . . . . . . . . . . . 137

$\begin{array}{ll}\text { List of References } & 151\end{array}$ 


\section{List of Tables}

2.1 General characteristics desired from wind turbine airfoils (adapted from Ref. $[17]) \ldots \ldots \ldots$

3.1 Key aerodynamic characteristics for the three airfoils. Numbers in brackets represent the percent change under forced transition . . . .

3.2 Sample $L_{16}$ Taguchi orthogonal array consisting of 4 parameters (A,

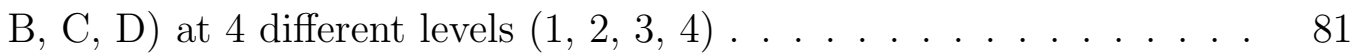

3.3 Parameters and associated levels for 24\%, 30\% and 36\% thick airfoils

4.1 Sample measured values and their associated uncertainties (95\% confidence) for the S809 and $C_{T}$ airfoils . . . . . . . . . . . . . . . 127 


\section{List of Figures}

1.1 Example of a flatback airfoil, note the blunt trailing-edge . . . . . . . 2

1.2 Typical 3-bladed, upwind horizontal axis wind turbine . . . . . . . 4

1.3 Horizontal-axis wind turbine growth in the USA since 1998. Adapted from $[15] \ldots \ldots \ldots \ldots \ldots \ldots$

1.4 Blade planform with airfoil cross-sections shown below . . . . . . . 5

2.1 Airfoil showing key identifying parameters . . . . . . . . . . . . . 10

2.2 Pressure distribution for a symmetric airfoil at $0^{\circ}$ and $10^{\circ}$ angle of attack 11

2.3 Pressure distribution for a cambered airfoil at $0^{\circ}$ and $10^{\circ}$ angle of attack 13

2.4 NACA4415 airfoil characteristics . . . . . . . . . . . . . 16

2.5 Lift-to-drag ratio- a measure of an airfoil's efficiency . . . . . . . 18

2.6 The aerodynamic loads acting on a wind turbine airfoil . . . . . . . . 19

2.7 NACA4415 airfoil torque and thrust characteristics . . . . . . . . . 20

2.8 The effect of changing the blade twist, $\beta$, on the airfoil's wind turbine operational efficiency at the $30 \%$ and $70 \%$ span locations . . . . . . 22

2.9 The coefficient of torque for an airfoil when drag effects are included and not included . . . . . . . . . . . . . . . . . 23

2.10 A wind turbine airfoil (left) that has been transformed into a flatback airfoil by truncation (middle) and thickening of the trailing-edge (right) 27 
2.11 Trailing-edge modifications used in an attempt to alleviate drag and noise effects; (a) Gurney flap, (b) Splitter plate, (c) Cavity, (d) Wavy trailing-edge . . . . . . . . . . . . . . . . . . . . . 28

2.12 Family of wind turbine specific airfoils designed by NREL [70] . . . . 33

2.13 Family of wind turbine specific FFA-W1-XXX (left) and FFA-W3-

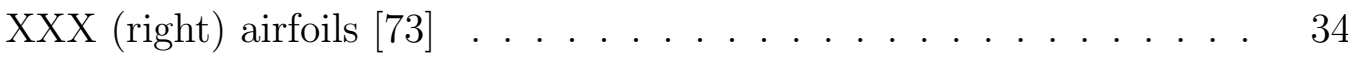

2.14 Family of wind turbine specific DUT airfoils [75] . . . . . . . . . . . 35

2.15 Family of wind turbine specific Riso-B1 airfoils [78] . . . . . . . . 36

2.16 Various examples of flatback airfoil designs (adapted from [31, 46, 59,

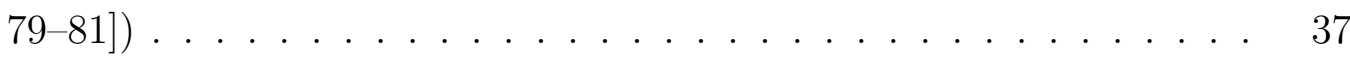

3.1 Example function . . . . . . . . . . . . . . . . . . 40

3.2 Example Pareto front showing the optimal evaluations given two objective functions. Note that since this is a minimization problem, smaller values are considered optimal _ . . . . . . . . . . . . . . . . . . . . . 42

3.3 Simplistic overview of flatback airfoil optimization software architecture 45

3.4 Simplified work-flow of a genetic algorithm . . . . . . . . . . . 46

3.5 An example of the intermediate crossover used in gamultiobj . . . . . 49

3.6 Bézier curve generation using 3 control points, and 25\% interpolation

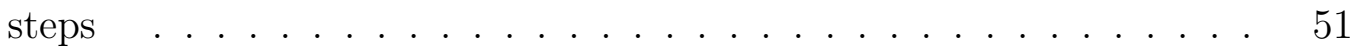

3.7 Bézier control points used to control the shape of the airfoil . . . . . 54

3.8 Sensitivity of $C_{L}$ Max and $C_{L}$ Min with respect to the number of panels used in XFOIL . . . . . . . . . . . . . . . . . . 60

3.9 Comparison of XFOIL lift predictions and experimentally measured values for S809 airfoil . . . . . . . . . . . . . . . . . 61

3.10 Comparison of XFOIL drag predictions and experimentally measured values for S809 airfoil . . . . . . . . . . . . . . . . . . 
3.11 Sample Pareto front showing an individual with the largest RMS value 62

3.12 Results of the convergence analysis when varying population size . . . 63

3.13 Results of the convergence analysis when varying number of generations 64

3.14 Three $30 \%$ thick airfoils optimized for maximum $C_{L} / C_{D}, C_{T}$, and $C_{T} / C_{N} 69$

$3.15 C_{L}$ of the airfoils under free transition conditions at $R e=6 \times 10^{6} \ldots 70$

$3.16 C_{L}$ of the airfoils under forced transition conditions at $R e=6 \times 10^{6} .70$

$3.17 C_{D}$ of the airfoils under free transition conditions at $R e=6 \times 10^{6} \ldots 71$

$3.18 C_{D}$ of the airfoils under forced transition conditions at $R e=6 \times 10^{6} .71$

$3.19 C_{L} / C_{D}$ of the three airfoils under free transition conditions at $R e=$ $6 \times 10^{6} \ldots \ldots \ldots \ldots \ldots \ldots \ldots$

$3.20 C_{L} / C_{D}$ of the three airfoils under forced transition conditions at $R e=$ $6 \times 10^{6} \ldots \ldots \ldots \ldots \ldots 74$

3.21 The $C_{L} / C_{D}$ weighted-sum (25\% free, $75 \%$ forced transition) performance of each of the three airfoils in the $\alpha$ range . . . . . . . . . . . . 74

$3.22 C_{T}$ of the three airfoils under free transition conditions at $R e=6 \times 10^{6} \quad 75$

$3.23 C_{T}$ of the three airfoils under forced transition conditions at $R e=6 \times 10^{6} 75$

3.24 The $C_{T}$ weighted-sum (25\% free, $75 \%$ forced transition) performance of each of the three airfoils in the $\alpha$ range . . . . . . . . . . . . 76

$3.25 C_{T} / C_{N}$ of the three airfoils under free transition conditions at $R e=$

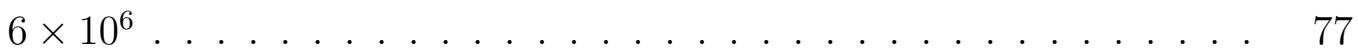

$3.26 C_{T} / C_{N}$ of the three airfoils under forced transition conditions at $R e=$

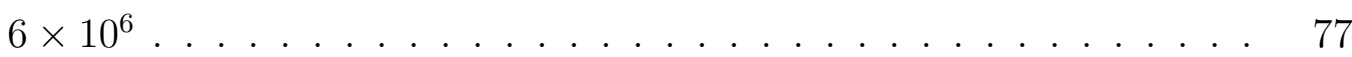

3.27 The $C_{T} / C_{N}$ weighted-sum (25\% free, $75 \%$ forced transition) performance of each of the three airfoils in the $\alpha$ range . . . . . . . . . . . 77

3.28 The definition of $A O A_{\text {range }}$; the distance of the upper limit of the $6^{\circ}$ optimization range to $C_{L M a x} \ldots \ldots \ldots \ldots$. . . . . . . 
3.29 The $C_{L \operatorname{Max}}$ (left) and $C_{L \text { ratio }}$ (right) as calculated by XFOIL at $R e=$ $6 \times 10^{6}$ under free transition of other wind turbine specific airfoils . . 82

3.30 The results of the sensitivity analysis using the Taguchi method for $24 \%, 30 \%$, and $36 \%$ thick airfoils. Note that in all cases, the y-axis shows the percent change relative to the average result for each respective thickness and configuration (free or forced transition) and the $\mathrm{x}$-axis is the value for each constraint/objective . . . . . . . .

3.31 The ratio of $C_{L \text { Max }}$ in forced transition compared to free transition for the CU airfoils in comparison to others . . . . . . . . . . . . 87

3.32 The CU-W1-XX airfoil family consisting of 6 airfoils, 21-36\% in thickness 90

3.33 The $C_{L \text { ratio }}$ (left) and $C_{L \text { Max }}$ (right) as calculated by XFOIL at $R e=$ $6 \times 10^{6}$ of the CU-W1-XX airfoils in comparison to other wind turbine specific airfoils . . . . . . . . . . . . . . . . . . . . . . . . . . 91

3.34 The average $C_{L} / C_{D}$ over the $6^{\circ}$ optimized range under free (left) and forced (right) transition conditions as calculated by XFOIL at $R e=$ $6 \times 10^{6}$ of the CU-W1-XX airfoils in comparison to other wind turbine specific airfoils . . . . . . . . . . . . . . . .

4.1 A schematic of the airfoil testing set-up in ME3224 at Carleton University. Note that schematic is not to scale. . . . . . . . . . . . 94

4.2 A sketch of the angle rod implemented in the experimental work. Note that the sketch is not to scale. . . . . . . . . . . .

4.3 Photograph of a manufactured airfoil, showing the EconoKote covering (dark blue), the foam (black), and the airfoil shaft (metallic) . . . . . 101

4.4 Schematic illustrating the momentum drag method. Note the bounds of the measurements used in the data reduction, as well as the averaged velocities outside the airfoil wake. . . . . . . . . . . . . . 
4.5 The effect of applying wind tunnel corrections to the measured lift data 108

4.6 The effect of applying wind tunnel corrections to the measured drag data . . . . . . . . . . . . . . . . . 108

4.7 The S809 airfoil used for wind tunnel set-up and method validation purposes, as well as the $C_{T}$ airfoil developed in this work, both of which were experimentally tested at Carleton University . . . . . . 109

4.8 The variation of $C_{D \text { Min }}$ and $C_{L \text { Max }}$ with respect to $R e$ as predicted by XFOIL for the CU-W1-30 airfoil . . . . . . . . . . . . . 110

4.9 The coefficient of lift results from Carleton University (CU) compared to Ohio State University (OSU) and XFOIL at $R e \doteq 750,000 \ldots \ldots 112$

4.10 The coefficient of drag results from Carleton University (CU) compared to Ohio State University (OSU) and XFOIL at $R e \doteq 750,000 \ldots \ldots 113$

4.11 The coefficient of lift-to-drag results from Carleton University (CU) compared to Ohio State University (OSU) and XFOIL at $R e \doteq 750,000114$

4.12 The coefficient of lift results from Carleton University (CU) compared to XFOIL at $R e \doteq 750,000 \ldots \ldots \ldots \ldots \ldots \ldots$

4.13 The coefficient of drag results from Carleton University (CU) compared to XFOIL at $R e \doteq 750,000 \ldots \ldots \ldots \ldots \ldots 17$. . . . . . . . 117

4.14 The coefficient of lift-to-drag ratio results from Carleton University (CU) compared to XFOIL at $R e \doteq 750,000 \ldots \ldots \ldots \ldots$

4.15 The coefficient of torque results from Carleton University (CU) compared to XFOIL at $R e \doteq 750,000 \ldots \ldots \ldots \ldots$ 


\section{Nomenclature}

\begin{tabular}{|c|c|c|}
\hline Symbol & Definition & Units \\
\hline$\alpha$ & Angle of attack & $\circ$ \\
\hline$\alpha_{C_{L} \operatorname{Max}}$ & Maximum lift angle of attack & $\circ$ \\
\hline$\alpha_{C_{L 0}}$ & Zero-lift angle of attack & $\circ$ \\
\hline$\beta$ & Local blade geometric twist & $\circ$ \\
\hline$\epsilon_{s b}$ & Solid blockage correction & - \\
\hline$\epsilon_{w b}$ & Wake blockage correction & - \\
\hline$\nu$ & Number of experimental repeats minus 1 & - \\
\hline$\omega$ & Rate of rotation & $\mathrm{rad} / \mathrm{s}$ \\
\hline$\phi$ & Flow angle & $\circ$ \\
\hline$\rho_{\infty}$ & Fluid density & $\mathrm{kg} / \mathrm{m}^{3}$ \\
\hline$\sigma$ & Constant used in wind tunnel corrections & - \\
\hline$A$ & Area & $m^{2}$ \\
\hline
\end{tabular}


$A_{\text {sect }} \quad$ Area of test section $\quad m^{2}$

AC Aerodynamic Center

AEP Annual Energy Production

AOA Angle of attack

$A O A_{\text {range }}$ Distance from upper end of optimization range to $C_{L}$ Max

$\circ$

BEM Blade Element Momentum theory

$B_{n, i}(z) \quad$ Bernstein polynomial used in Bézier curve

BSDS Blade System Design Study

C Airfoil Chord

$C_{D} \quad$ Coefficient of drag

$C_{D \text { Min }} \quad$ Minimum coefficient of drag

$C_{D u} \quad$ Uncorrected coefficient of drag

CFD Computational Fluid Dynamics

$C_{L} \quad$ Coefficient of lift

$C_{L} / C_{D} \quad$ Coefficient of lift-to-drag ratio

$C_{L} \operatorname{Max} \quad$ Maximum coefficient of lift

$C_{L \text { ratio }} \quad$ Ratio between clean and rough maximum lift performance

$C_{L u} \quad$ Uncorrected coefficient of lift 


\begin{tabular}{|c|c|c|}
\hline$C_{M}$ & Coefficient of pitching moment & - \\
\hline$C_{M u}$ & Uncorrected coefficient of moment & - \\
\hline$C_{N}$ & Coefficient of thrust & - \\
\hline$C_{P}$ & Coefficient of pressure & - \\
\hline$C_{T}$ & Coefficient of torque & - \\
\hline$C_{T} / C_{N}$ & Coefficient of torque-to-thrust ratio & - \\
\hline $\mathrm{CU}$ & Carleton University & - \\
\hline$D$ & Drag force & $\mathrm{N}$ \\
\hline DUT & Delft University of Technology & - \\
\hline$e^{N}$ & Transitional model employing the amplication factor, $\mathrm{N}$ & - \\
\hline FFA & Aeronautical Research Institute of Sweden & - \\
\hline$g$ & Gravity & $m / s^{2}$ \\
\hline$h$ & Maximum airfoil camber & $\%$ \\
\hline$H$ & Height difference in manometer reading & $\mathrm{m}$ \\
\hline HAWT & Horizontal Axis Wind Turbine & - \\
\hline$H t$ & Test section height & $\mathrm{m}$ \\
\hline$I_{X X}$ & Sectional moment of inertia & $m^{4}$ \\
\hline$J$ & Torsional stiffness constant & $m^{4}$ \\
\hline
\end{tabular}


$K \quad$ Constant used in solid blockage relation

$L \quad$ Lift force $\quad$ N

$\dot{m} \quad$ Mass flow rate $\mathrm{kg} / \mathrm{s}$

$\begin{array}{lll}M & \text { Pitching moment } & \mathrm{Nm}\end{array}$

Ma Mach number

$n \quad$ Number of control points minus 1 used in Bézier curve

N Thrust force $\quad$ N

NREL National Renewable Energy Laboratory

NSGA-II Nondominated Sorting Genetic Algorithm

numvar Number of variables in genetic algorithm

Obj Number of objectives in genetic algorithm

OSU Ohio State University

$P \quad$ Pressure $\quad P a$

$\begin{array}{lll}P_{\infty} & \text { Free-stream pressure } & \mathrm{Pa}\end{array}$

Paero Aerodynamic power W

$P_{i} \quad$ Current control point used in Bézier curve

$\mathrm{P}(\mathrm{z}) \quad$ Point lying along Bézier curve

$r \quad$ Local radial location along blade

$\mathrm{m}$ 
R Resultant performance outcome used in Taguchi method

Re Reynolds number

$R e_{u} \quad$ Uncorrected Reynolds number

RMS Root Mean Square

$R_{x} \quad$ Random error

$S_{x} \quad$ Systematic error

$t \quad$ Maximum airfoil thickness

$\%$

$\mathrm{T}$

Torque force

$\mathrm{N}$

TE Trailing-edge thickness

$\%$

$t_{\nu, 95 \%} \quad$ Student t-Distribution at $95 \%$ confidence

Tu Absolute freestream turbulence value

$\%$

$U_{x} \quad$ Uncertainty in experimental data

V Velocity

$m / s$

$V_{\infty} \quad$ Oncoming wind velocity

$m / s$

$V_{\text {eff }} \quad$ Effective (resultant) velocity

$m / s$

$V_{\text {rot }} \quad$ Velocity due to rotation

$m / s$

Vol Volume of wind tunnel model

$m^{3}$

weight Weighting used in optimization scheme for clean performance

$\%$ 


$\begin{array}{lll}\mathrm{x} / \mathrm{C} & \text { Position along chord } & \% \\ X_{C P} & \text { Center of pressure } & \% \\ x_{i}^{H, V} & \text { Control point, and horizontal or vertical direction of movement } & - \\ \bar{y} & \text { Neutral line of airfoil } & - \\ y_{l} & \text { y-coordinate of lower surface } & - \\ y_{u} & \text { y-coordinate of upper surface } & - \\ z & & \text { Interpolator used in Bézier curve }\end{array}$




\section{Chapter 1}

\section{Introduction and Motivation}

A better understanding and awareness of global warming has urged nations around the world to reduce emissions by means of green energy alternatives. Today's liftdriven wind turbines are a promising platform for utility scale energy production thanks to their low cost-of-energy. In an attempt to capture more energy from the wind, the current trend of Horizontal Axis Wind Turbines (HAWTs) is to increase in diameter, particularly in offshore applications where high wind speeds offer the potential for large energy capture [1-3]. The theoretical aerodynamic power of a wind turbine, as shown in Equation 1.1, is a function of the density, $\rho_{\infty}$, the area, $A$, and the oncoming wind velocity, $V_{\infty}$. Since $A=\pi r^{2}$, the aerodynamic power is shown to increase with the square of the rotor radius. Furthermore, the increased Reynolds numbers that come with the larger chord lengths of longer blades generally results in better aerodynamic performance and in turn, slightly superior aerodynamic efficiency of the wind turbine $[4,5]$. These long wind turbine blades, sometimes exceeding $80 \mathrm{~m}$ [6], have introduced several structural challenges due to the increased weight and aerodynamic forces acting on the blade. For this reason, the use of flatback airfoils- airfoils with a thick or blunt trailing-edge as shown in Figure 1.1particularly in the inboard regions of the wind turbine rotor where structural integrity 
is paramount, has been proposed and implemented by certain manufacturers such as Siemens, Enercon, Mitsubishi Heavy Industries and General Electric [7-10]. Flatback airfoils offer a balance of good structural properties while maintaining or in cases, improving the aerodynamic properties, which allows for lighter and longer blades to be produced $[11,12]$.

$$
P_{\text {aero }}=\frac{1}{2} \rho_{\infty} A V_{\infty}^{3}
$$

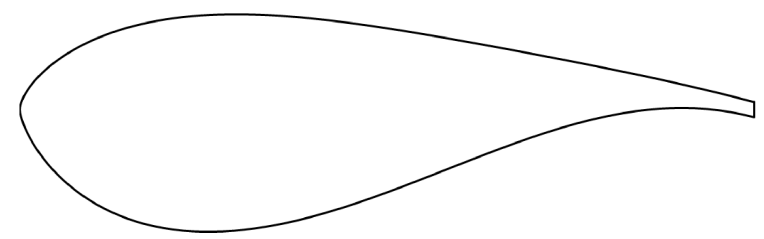

Figure 1.1: Example of a flatback airfoil, note the blunt trailing-edge

As the first link between the conversion of the wind's kinetic energy into mechanical energy to drive the wind turbine generator, proper airfoil design and optimization are paramount in the design of an efficient wind turbine. The high structural demands of today's long rotor blades, require the use of relatively thick airfoils, thereby eliminating the possibility of adapting existing thin airfoils designed for aerospace applications. Furthermore, the relatively low Mach numbers which wind turbines experience, once again rule-out the possibility of using airfoils designed for the aerospace industry where Mach numbers are generally higher. For these reasons, manufacturers and researchers design and optimize airfoils specifically with a wind turbine application in mind in order to maximize their aerodynamic and structural potential. 


\subsection{Current State of Horizontal-Axis Wind Tur- bines}

Today's wind turbines are most commonly in an upwind configuration and consist of a three-bladed rotor which rotates about a horizontal-axis. The number of blades on a rotor is a balance between aerodynamic and structural efficiency, noise, and cost [13]. With fewer blades, the rotor must spin at faster speeds in order to achieve its optimal aerodynamic efficiency. The higher speeds, result in larger aerodynamic forces which in turn require a structurally stronger blade construction. Furthermore, the increased tip-speed of the rotor leads to larger noise emissions- a problem particularly in populated areas [14]. That being said, by having fewer blades, the costs associated to the manufacture and transportation of the blades can be reduced, thereby lowering the overall cost of the turbine. Conversely, a greater number of blades allows for reduced aerodynamic loading and noise, but greater manufacturing and transportation costs. For these reasons, it has been found that three blades offer an ideal combination of the aerodynamic and structural efficiency, noise, and cost.

As illustrated in Figure 1.2, each blade connects to the rotor hub which turns the generator housed in the nacelle via a transmission shaft. Contained inside the hub are several actuators which allow the individual blades to pitch in order to ensure that they are operating under ideal conditions at all times. The nacelle is tilted slightly upwards by about 5 degrees, and sits atop the tower, commonly constructed from concrete or steel. This tilt ensures that the blades do not strike the tower, even when they are deformed from the aerodynamic forces of the wind. In order to maximize the power output, the wind turbine nacelle and rotor yaws about the tower axis in an attempt to face the wind straight on. 


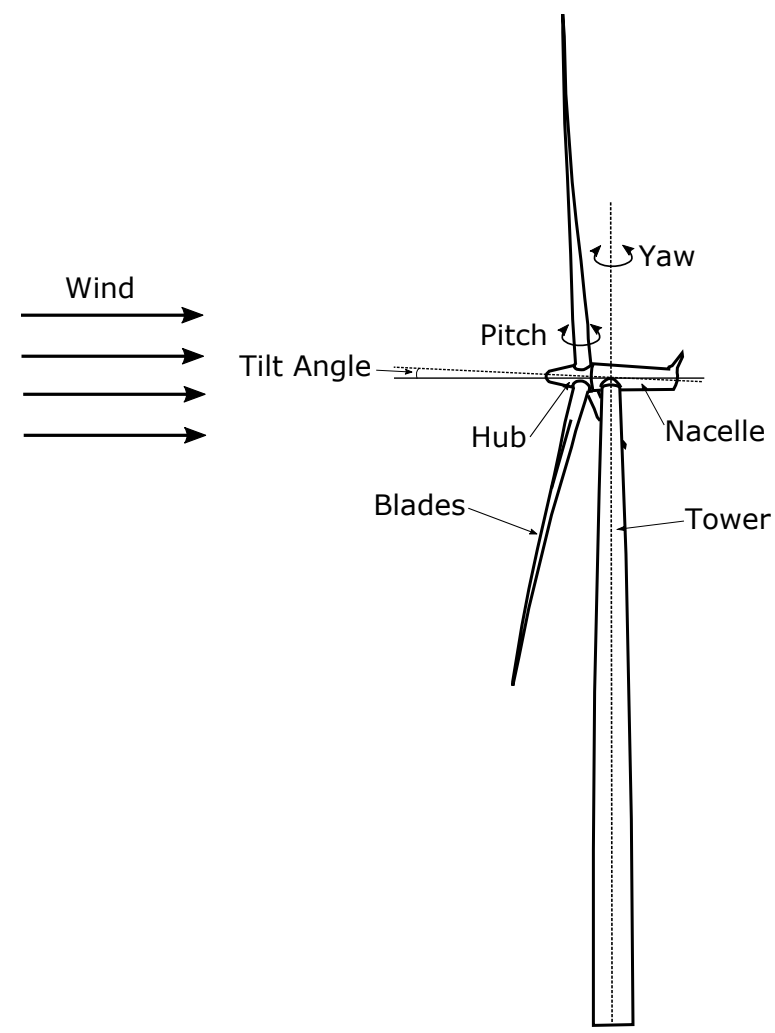

Figure 1.2: Typical 3-bladed, upwind horizontal axis wind turbine

As shown in Figure 1.3, both the rotor diameter and hub height have been steadily increasing throughout the years with the average in 2014 of installed wind turbines in the United States of America being almost $100 \mathrm{~m}$ and over $80 \mathrm{~m}$, respectively [15]. The increase in rotor diameter allows for a larger amount of energy to be captured per wind turbine thereby reducing the cost-of-energy of wind power [16]. The higher average hub heights allow the turbine to harvest the more powerful winds found at higher levels above the ground, as well as accommodate the ever-increasing blade lengths. On average, a single HAWT produces approximately $2 \mathrm{MW}$ of energy, however, certain models, particularly those used in offshore applications, are capable of $8 \mathrm{MW}$ capacities. 


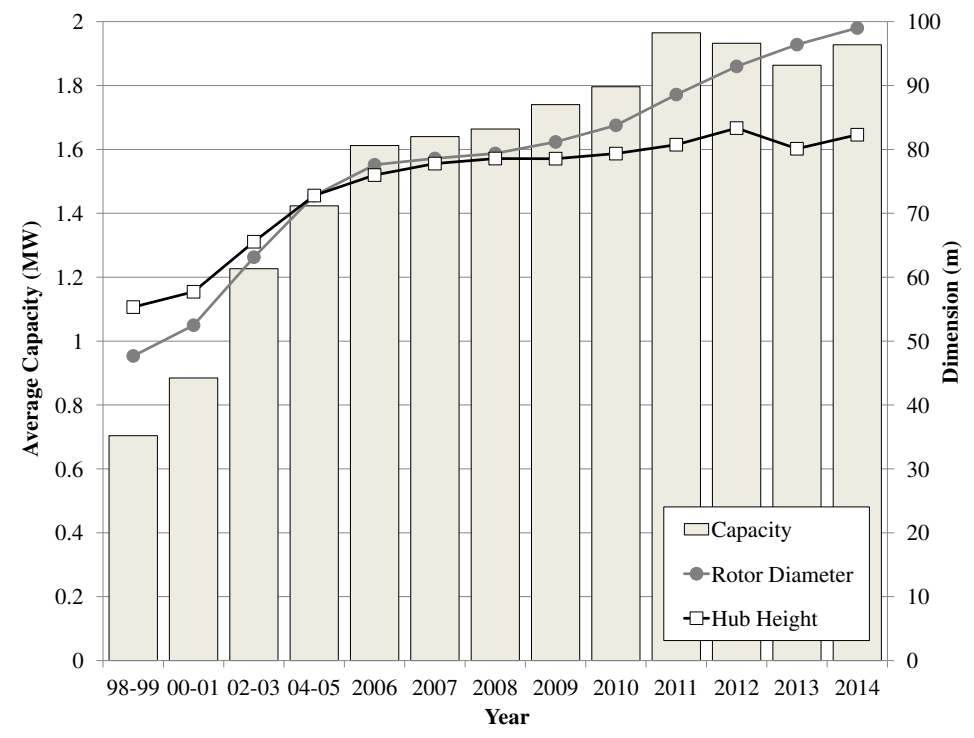

Figure 1.3: Horizontal-axis wind turbine growth in the USA since 1998. Adapted from $[15]$

\subsection{Aerodynamics of Horizontal-Axis Wind Tur-}

\section{bines}

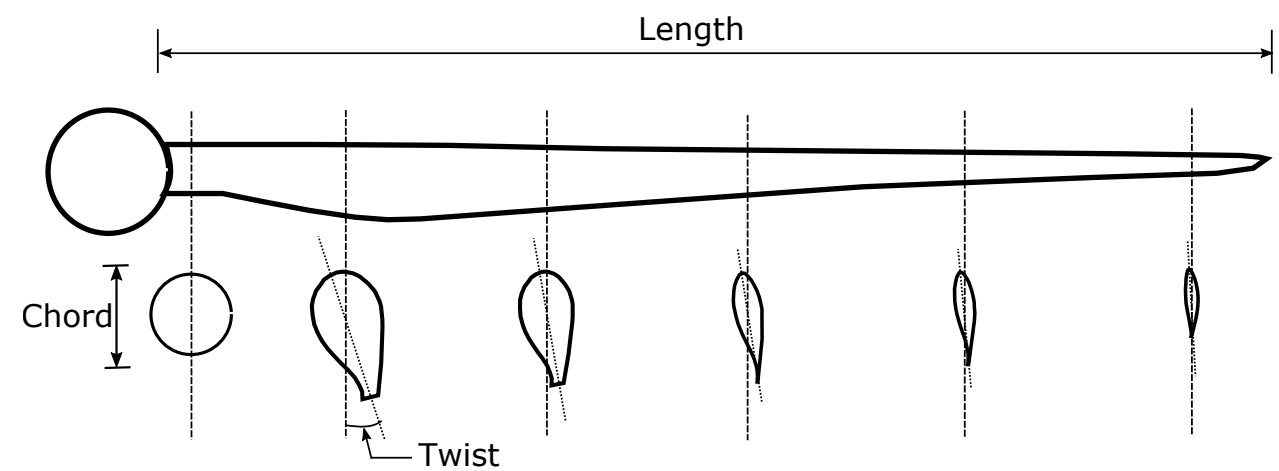

Figure 1.4: Blade planform with airfoil cross-sections shown below

As shown in Figure 1.4, HAWT blades consist of several different airfoil profiles with chord lengths and twist angles that vary along its length. The airfoil design and placement are optimized to achieve the desired aerodynamic performance, all 
while maintaining structural integrity. The principal aerodynamic forces acting on a wind turbine include the torque and thrust forces acting in the plane of rotation and normal to the plane of rotation, respectively. Torque forces drive the rotation of the rotor, and in turn energy production, whereas thrust forces tend to bend the rotor blades and tower in the direction of the wind.

As can be seen in Figure 1.4, at the root of the blade, a cylindrical cross-section is used such that the extremely large loads experienced by the blade can be tolerated and so that the blades can easily be pitched. At the inner region of the blade, thick airfoil profiles are chosen due to their structural properties as well as to ensure a smooth transition from the cylindrical section. It is in this inner region where thick, flatback airfoils are typically found thanks to their high lift characteristics, which allow for maximum rotational torque, as well as their favorable structural characteristics. In the mid-span region of the blade, airfoils are generally chosen based on their aerodynamic performance, particularly their lift-to-drag ratio, while once again ensuring a smooth transition in shape from the thick inboard airfoils. At the tip region, thinner airfoils are chosen based on their low noise levels [17] and high aerodynamic efficiency.

The low magnitude of rotational velocity at the inboard regions of the blade results in high angles of attack, therefore, high levels of geometric twist must be incorporated into the blade design such that the airfoil operates at its intended design conditions. At further distances from the hub, the component of rotational velocity increases thereby decreasing the effective angle of attack, and in turn reducing the magnitude of geometric twist required. Similarly, in order to maintain a roughly constant aerodynamic loading along the length of the blade, the chord- the distance between the leading and trailing-edge of the airfoil in cross-section- must be adjusted [1]. At the inboard region of the blade, large chord lengths are found in an attempt to produce more lift force from the relatively low effective velocities. The chord length 
tapers along the blade as it nears the tip due to the increasing effective velocities which produce greater aerodynamic forces. The tip itself forms a point-like figure in order to minimize tip-vortices which will act to reduce the overall power by imparting downwash on the blade.

One can note that in Figure 1.4, the maximum chord length does not in fact lie at the inner-most region of the blade. The reason for this is primarily due to manufacturing and transportation constraints. In order to ensure roughly equal aerodynamic loading at all sections along the blade length, extremely large chord lengths would be necessary in the inboard regions due to the low component of rotational velocity. These large sections are a challenge to manufacture due to the large molds that would be necessary, as well as the high levels of twist that are required [1]. Furthermore, the large chord lengths and twist cause issues when transporting the blade, particularly in regions where underpasses, tunnels, or bridges are encountered [18]. A cylindrical section at the root allows for a simplistic pitch mechanism, as well as an easier to construct, and transport blade design. The manufacturer Enercon, produces an aerodynamic shell which is manufactured and transported separately, and is attached to the cylindrical section on-site in an attempt to alleviate some of these issues [19].

\subsection{Motivation}

Although the design of wind turbine airfoils has been performed before by means of the designer's experience, or optimization tools, no studies, to the author's knowledge, have been performed to examine how the specified design criteria and parameters affect the airfoil shape and performance. Common design criteria for wind turbine airfoils include insensitivity to leading-edge roughness, good structural properties, smooth stall characteristics, low noise, high lift-to-drag ratio, high coefficient of torque 
and more.

Airfoils with a thick trailing-edge, referred to as flatback airfoils, have been shown to offer beneficial aerodynamic and structural characteristics, particularly in the inboard regions of rotor blades where the velocities are low, and the structural requirements are high. The thick trailing-edge not only increases the sectional area and moment of inertia, but also increases the lift characteristics and insensitivity to roughness of the airfoil in comparison to traditional sharp trailing-edge airfoils.

A numerical optimization tool, which combines both aerodynamic and structural properties, allows for the design of efficient airfoils to be used on large wind turbine blades. Rather than using existing, off-the-shelf airfoils, airfoils with specific user-defined properties can be custom designed which ensures that ideal operating characteristics are met for a given wind turbine. The use of numerical optimization tools ensure that for each design case, hundreds or even thousands of designs are evaluated, such that the global optimal condition is achieved.

Using an airfoil design tool developed in this work, several flatback airfoils will be numerically optimized under varying aerodynamic evaluators, such as lift-to-drag ratio, torque-to-thrust ratio, and torque coefficient. The airfoils will be characterized and compared in order to determine how different aerodynamic evaluators effect the airfoil shape and performance. Further analysis will be conducted to examine the effect of other airfoil design parameters using a Taguchi method [20]. An airfoil family, ranging in thicknesses will be designed using the acquired knowledge.

A wind tunnel experimental set-up will be developed and used to test select airfoils in order to validate the numerical performance predictions, and highlight any discrepancies. The outcome of this study is the development of a numerical optimization tool used to determine the ideal airfoil for a given set of operating conditions and requirements, as well as the effect that the selected aerodynamic evaluator, and other 
design parameters, have on the airfoil design and performance. Furthermore, wind tunnel experiments will provide further insight into any differences between numerical and experimental analyses, as well as act to emphasize the importance of wind tunnel testing as part of the design process.

\subsection{Outline}

This thesis consists of five chapters. Chapter 2 will provide the reader with the necessary fundamentals and background information required for the understanding and interpretation of airfoil performance characteristics. Furthermore, it will highlight major studies that have been performed in relation to the design of wind turbine airfoils, as well as the benefits of flatback airfoils with wind turbine applications in mind. Chapter 3 will describe the foundations of numerical optimization techniques, the implementation of optimization techniques for the design of flatback airfoils, as well as the numerical performance predictions of the optimized airfoils. Chapter 4 will outline the experimental set-up used for the wind tunnel analysis of airfoils, and provide a comparison of the experimental and numerical data. Finally, Chapter 5 will summarize the findings of the thesis, suggest future work, and conclude the thesis. 


\section{Chapter 2}

\section{Literature Review and Background}

\subsection{Aerodynamics of Airfoils}

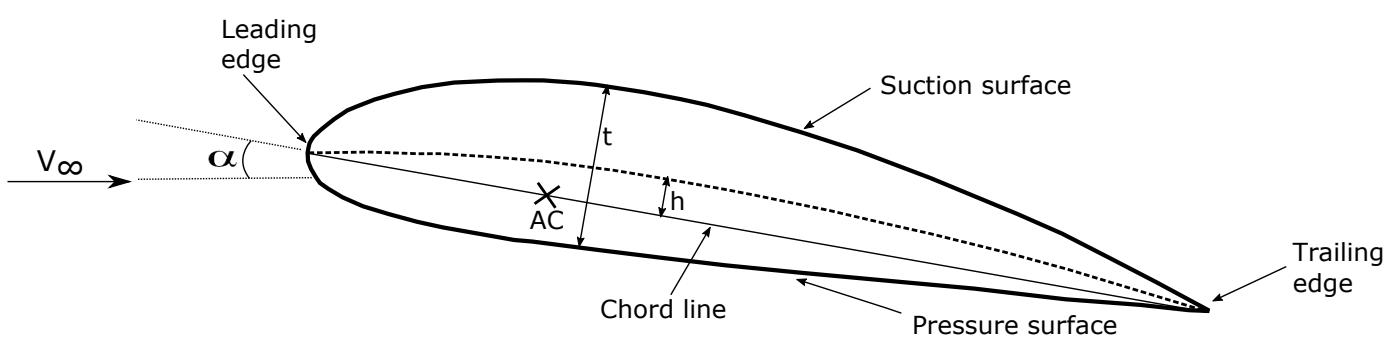

Figure 2.1: Airfoil showing key identifying parameters

An airfoil is the cross-sectional shape of a wing, propeller, sail, or blade. This aerodynamically tailored shape, as illustrated in Figure 2.1, consists of a upper and lower surface- commonly referred to as the suction and pressure surfaces, respectively. The chord of the airfoil is the distance between the leading and trailing-edge, and the thickness refers to the distance between the pressure and suction surfaces. The camber line, or mean line, defines the asymmetry between the pressure and suction surfaces. Generally, both the maximum thickness and maximum camber, denoted by $t$ and $h$, respectively, are used in the identification of airfoils and are expressed as a percentage

of the chord length. The aerodynamic center, $\mathrm{AC}$, is denoted by an $X$ in Figure 2.1, 
and by convention is typically located at the $25 \%$ chord position. Furthermore, the location of the maximum thickness and maximum camber are defined as the distance, as a percentage of the chord, from the leading-edge and are also commonly used in the identification and characterization of an airfoil. In the case of flatback airfoils, the relative thickness of the trailing-edge is also an important characterizing feature. For the NACA4418 airfoil shown in Figure 2.1, a maximum thickness of 18\% is found at the $30 \%$ chord location and a maximum camber of $4 \%$ is found at the $40 \%$ chord location. The airfoil's chordline forms an angle with the vector of the oncoming flow velocity; this angle, $\alpha$, is referred to as the angle of attack (AOA).
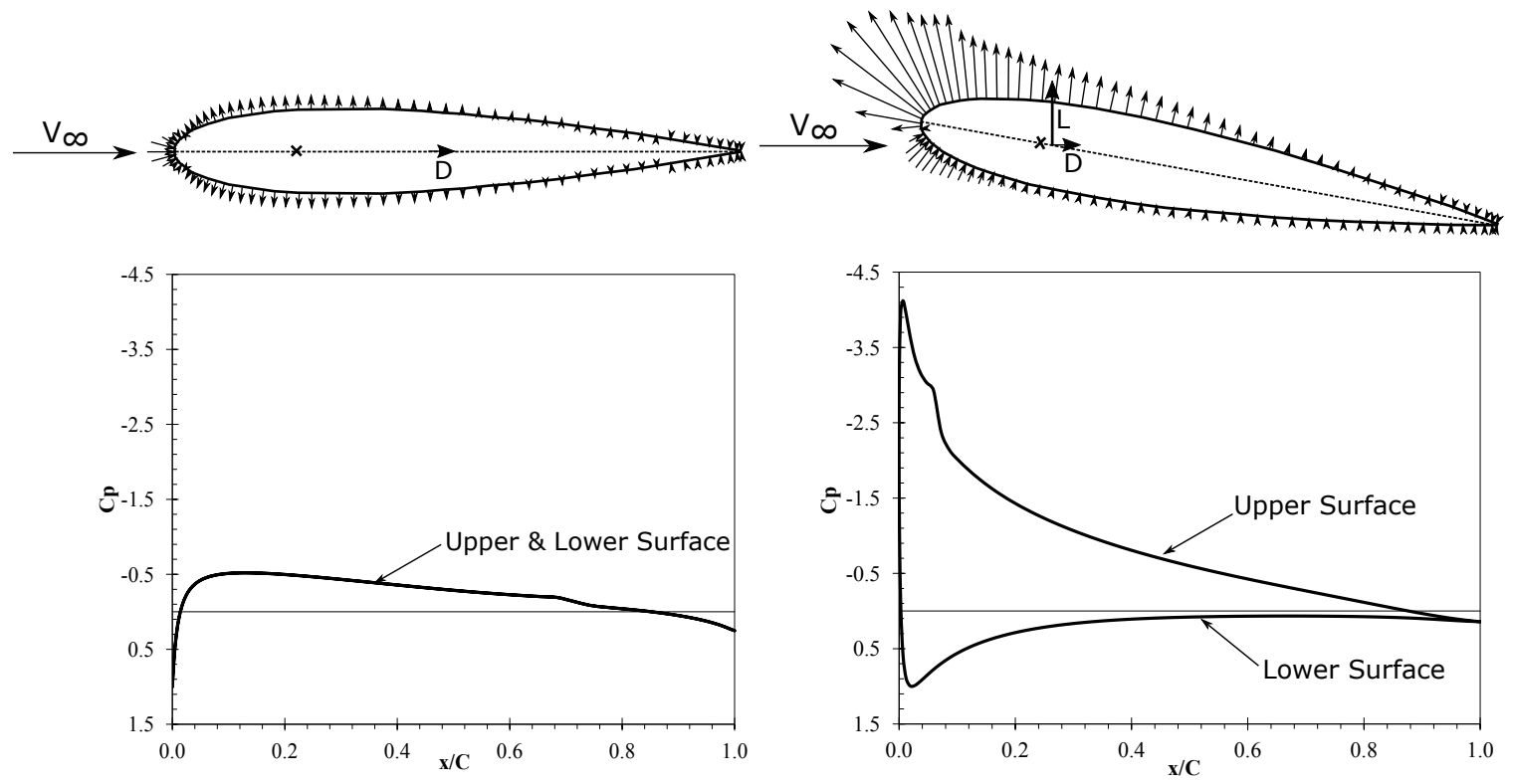

(a) $\alpha=0^{\circ}$

(b) $\alpha=10^{\circ}$

Figure 2.2: Pressure distribution for a symmetric airfoil at $0^{\circ}$ and $10^{\circ}$ angle of attack

The aerodynamic forces acting on an airfoil come as a result of the acceleration and deceleration of the flow over the surface of the airfoil, which in turn results in pressure forces acting normal to the outer surface of the airfoil. As the fluid molecules 
are forced around the contour of the airfoil, their velocity and pressure changes in accordance to Bernoulli's equation (assuming steady, inviscid, incompressible flow) [21]. At zero degrees of angle of attack, as seen in Figure 2.2(a), the symmetry of the airfoil, that is an airfoil with no camber, results in both the pressure and suction surfaces to have equal coefficients of pressure, $C_{P}$, as defined in Equation 2.1. It should be noted that the $C_{P}$ axis in Figure 2.2, is reversed such that the negative values typically found on the suction surface of the airfoil, are oriented above the positive values typically found on the pressure surface. This is done by convention to ease the visualization of the pressure over the airfoil cross-section.

$$
C_{P}=\frac{P-P_{\infty}}{\frac{1}{2} \rho_{\infty} V_{\infty}^{2}}
$$

where $\mathrm{P}$ is the pressure at the point of evaluation, $P_{\infty}$ is the free-stream pressure, $\rho_{\infty}$ is the fluid density and $V_{\infty}$ is the fluid velocity. The identical pressure profiles over the top and bottom surfaces of the symmetrical airfoil at zero degrees AOA results in a net-zero pressure force in the direction normal to the chord. The stagnation point of the flow, corresponding to $C_{P}=1$, can be seen in Figure 2.2(a) to occur at the leading-edge of the airfoil.

At non-zero angles of attack, in this case positive $10^{\circ}$ as shown in Figure 2.2(b), the stagnation point moves to the pressure side of the airfoil, just downstream from the leading-edge. Furthermore, the flow over the airfoil results in a different coefficient of pressure profile over the suction and pressure surfaces. As the flow accelerates over the leading-edge of the suction surface, large negative values of coefficient of pressure develop which then gradually reduce in velocity, and in turn increase in pressure. Conversely, as the flow decelerates over the leading-edge of the pressure surface, large positive values of coefficient of pressure are produced until the stagnation point, at 
which point the flow accelerates resulting in a decrease in pressure. The combination of the positive pressure on the lower, pressure surface, and the negative pressure over the upper, suction surface, results in a net force acting upwards called lift. The point at which this net force acts is referred to as the center of pressure $\left(X_{C P}\right)$ and may change with respect to the angle of attack. The direction of the lift force always acts perpendicular to the oncoming flow, $V_{\infty}$, no matter the angle of attack. For airfoils with a sharp trailing-edge, the upper and lower surface pressures must be equal at the trailing-edge according to the Kutta condition [22]. Cambered airfoils, as shown in Figure 2.3, produce lift even at zero degrees of angle of attack due to the inherent asymmetry between the pressure and suction surfaces which results in a net positive lift force.

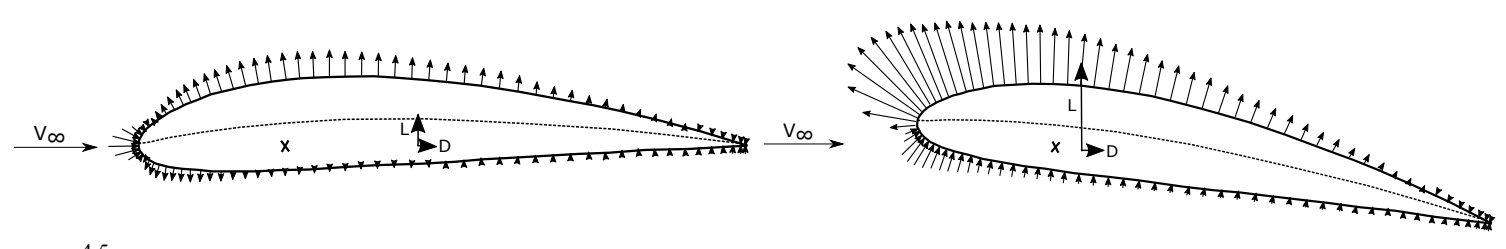

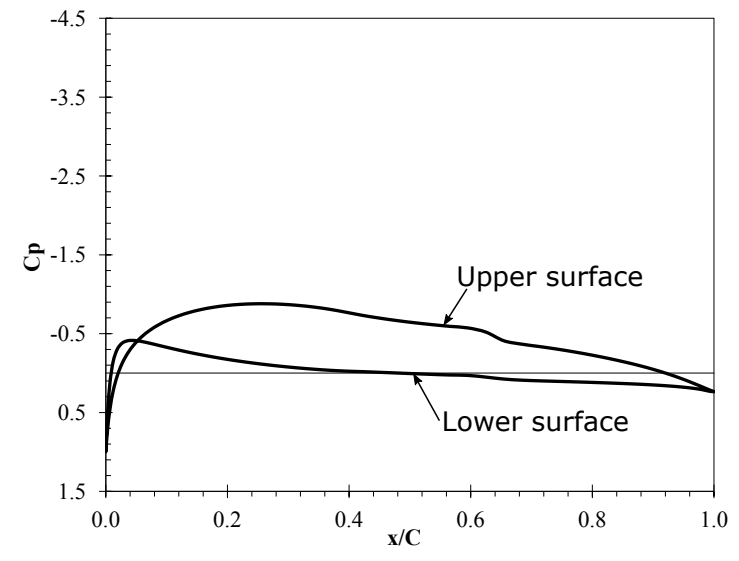

(a) $\alpha=0^{\circ}$

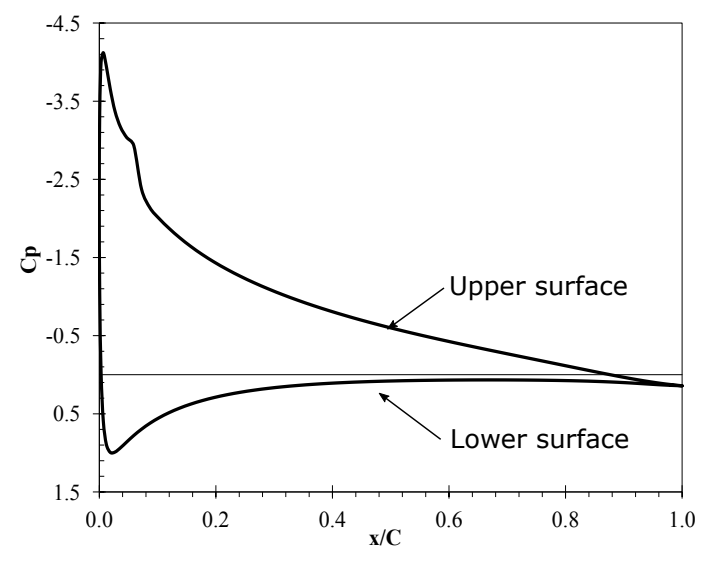

(b) $\alpha=10^{\circ}$

Figure 2.3: Pressure distribution for a cambered airfoil at $0^{\circ}$ and $10^{\circ}$ angle of attack

Since the lift force acts at the center of pressure, which is located a certain distance from the aerodynamic center, it creates a pitching moment on the airfoil. Depending 
on the magnitude of the lift and drag, and the distance of the center of pressure from the aerodynamic center, different moment forces will be produced at each angle of attack. Airfoils typically possess negative, nose-down, moment forces at positive angles of attack.

In addition to the lift force produced by the airfoils, the pressures forces also produce drag. This drag force always acts in the direction of the flow velocity, $V_{\infty}$, no matter the angle of attack. Pressure drag occurs due to net pressure differences acting in the direction of flow velocity. The stagnation point near the leading-edge results in high pressure region, whereas towards the trailing-edge, lower pressures regions are present which produces a net-rearward force. This pressure difference comes as a result of the form of the airfoil, and is greatly affected by flow separation. Since this force opposes the motion of the airfoil, it is considered to be a loss, or an inefficiency in the performance of the airfoil.

The discussion thus far has assumed inviscid and incompressible flow. The latter is for the most part a fair assumption for wind turbine airfoils where maximum tip speeds are generally around $70 \mathrm{~m} / \mathrm{s}(M a=0.21)$ [23], although current research is exploring tip speeds up to $110 \mathrm{~m} / \mathrm{s}(M a=0.33)[24]$. The assumption that the flow is inviscid, however, is not valid in real-world scenarios. The viscid flow near the surface of the airfoil creates a boundary layer which can greatly alter the aerodynamic performance of the airfoil due to flow separation, skin friction drag and more.

When the flow within the boundary layer over an airfoil encounters an adverse pressure gradient (i.e. where the pressure increases in the direction of the flow) it has a high tendency to separate due to flow reversal. This flow reversal comes as a result of the kinetic energy of the flow no longer being able to overcome the positive pressure gradient which is driving the fluid in the direction opposite to the flow. Flow separation occurs predominantly at high angles of attack when large adverse pressure 
gradients form, thereby rendering the rear portion of the suction surface of the airfoil ineffective, and resulting in a change in the effective shape of the airfoil. In turn, the total lift contribution of the airfoil reduces and a large increase in drag is created. The associated decrease in lift, and increase in drag, that comes as a result of flow separation, is termed stall. High Reynolds numbers tend to maintain higher inertial forces within the boundary layer, thereby delaying the onset of flow separation relative to lower Reynolds numbers.

$$
\begin{gathered}
C_{L}=\frac{L}{\frac{1}{2} \rho_{\infty} V_{\infty}^{2} C} \\
C_{D}=\frac{D}{\frac{1}{2} \rho_{\infty} V_{\infty}^{2} C} \\
C_{M}=\frac{M}{\frac{1}{2} \rho_{\infty} V_{\infty}^{2} C^{2}}
\end{gathered}
$$

The lift, drag and moment forces acting on a 2D airfoil cross-section are often nondimensionalized as seen in Equations 2.2-2.4. Figure 2.4 shows plots of the coefficient of lift, drag, moment and location of transition as predicted by the numerical solver XFOIL [25]- to be discussed in greater detail in subsequent chapters. The coefficient of lift and drag, $C_{L}$ and $C_{D}$, are seen to remain approximately linear up until a certain angle of attack, in this case about $13^{\circ}$, at which point a decrease in lift, and increase in drag can be observed. This corresponds to the stall angle of the airfoil. Before stall, the flow over the airfoil remains attached meaning that littleto-no separation has occurred. The airfoil behaves approximately according to thin airfoil theory, which assumes inviscid flow, and states that the slope of the lift curve is equal to $2 \pi / \mathrm{rad}^{-1}$ or $0.11 /^{\circ}$ [22]. In the linear region of the lift curve, viscous 


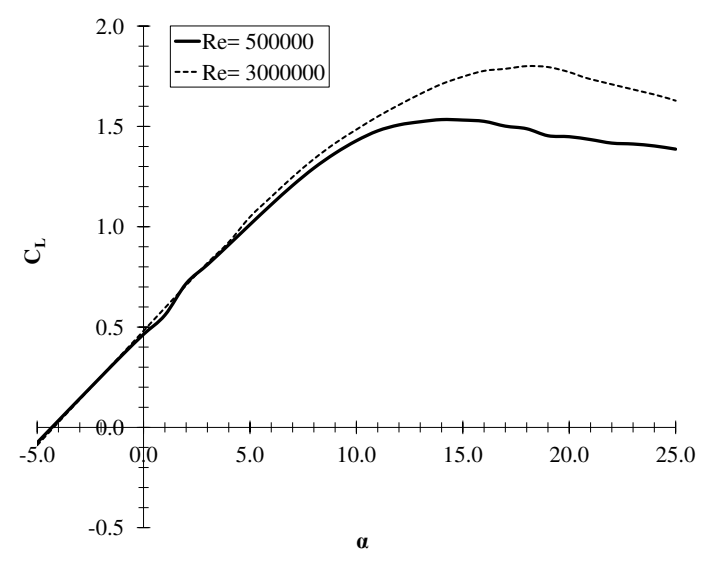

(a) Coefficient of lift

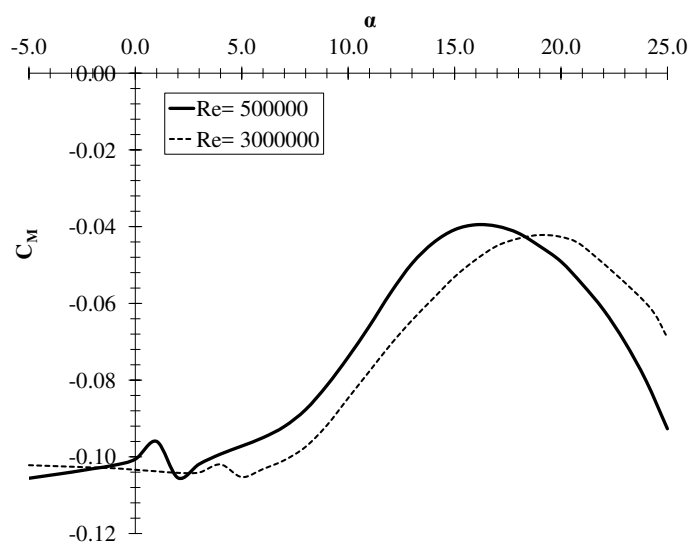

(c) Coefficient of moment

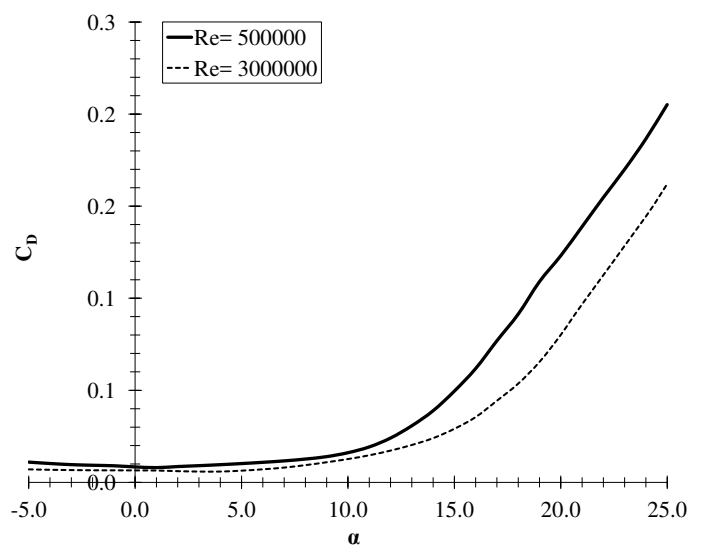

(b) Coefficient of drag

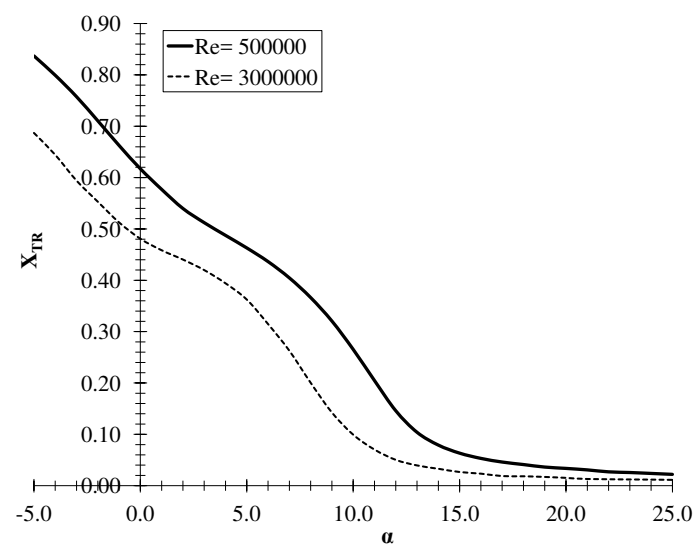

(d) Upper surface transition

Figure 2.4: NACA4415 airfoil characteristics

effects are rather minimal therefore thin airfoil theory is found to agree very well, however, around $13^{\circ}$ viscous effects begin to become more evident. Post-stall, the coefficient of drag increases dramatically due to the increase in pressure drag that comes as a result of flow separation. At the higher Reynolds number, an increase in maximum $C_{L}$, and a delay in stall occurs due to the higher energy levels within the boundary layer which aids in keeping the flow attached for longer. Furthermore, at high Reynolds numbers the boundary layer thins which leads to a reduction in profile drag, and in turn $C_{D}$ [26]. 
The coefficient of moment is seen to remain negative for all angles of attack due to the center of pressure always remaining aft of the aerodynamic center. As the angle of attack increases, the center of pressure gradually moves forward, toward the leading-edge of the airfoil. The combination of the lift and drag force acting at a certain distance from the aerodynamic center, results in a slight variation of the pitching moment, albeit negative.

The transition-to-turbulence point on the suction surface of the airfoil, as predicted by XFOIL, is seen to continually move forwards towards the leading-edge. This is due to an increase in pressure gradients near the leading-edge region which causes an earlier onset of transition to turbulence. Eventually, the transition point will remain relatively constant, and will occur very close to the leading-edge of the airfoil. At higher Reynolds numbers, transition occurs earlier due to the higher sensitivity of the flow to becoming turbulent.

The efficiency of the airfoil can be described as the ratio between the lift and drag forces, $C_{L} / C_{D}$. A highly efficient airfoil will produce high levels of lift, with relatively low levels of drag. The efficiency of the airfoil is typically plotted against the angle of attack as seen in Figure 2.5(a), or with the lift plotted against the drag as seen in Figure 2.5(b). For the sample plot shown in Figure 2.5(a), the maximum efficiency is observed to occur at approximately $6^{\circ}$ and reaches a higher maximum value at the higher Reynolds number due to the increased lift and decreased drag. The alternative method of presenting the airfoil efficiency, shown in 2.5(b), shows that for a given drag value, the lift values are greater in magnitude with the higher Reynolds number. In this plot, a sharp change in direction of the curve caused by the rapid onset of stall is apparent. The lift and drag values corresponding to the maximum efficiency of an airfoil can be determined from this plot by connecting a line, starting from the origin which is tangent to the airfoil drag polar. In this case, the maximum efficiency can be 


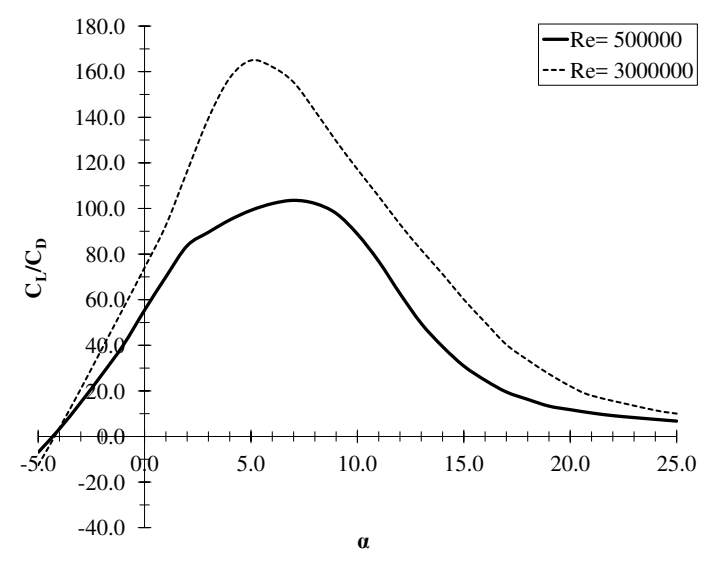

(a) Lift-to-drag ratio

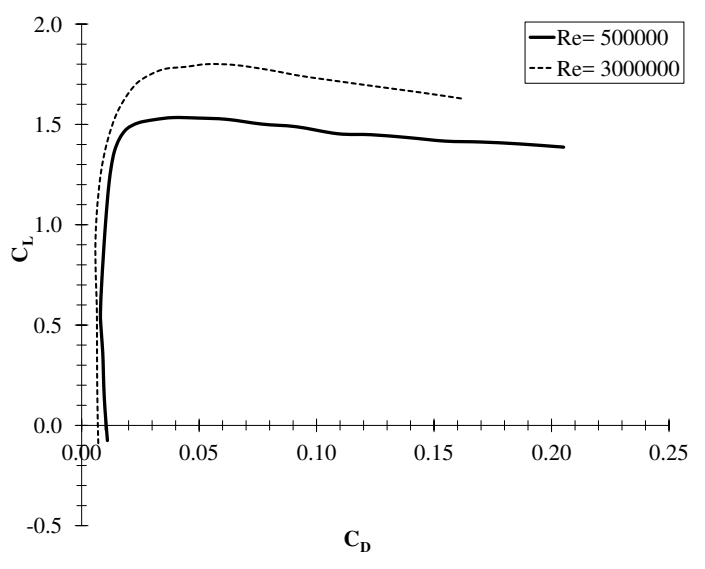

(b) Lift vs. drag

Figure 2.5: Lift-to-drag ratio- a measure of an airfoil's efficiency

found to occur near $C_{L}=1.2$ which corresponds to about $6^{\circ}$ (as confirmed in Figure 2.5(a)). Generally, as the overall thickness of an airfoil increases, the $C_{L} / C_{D}$ of the airfoil will decrease [27] due to an inherent large increase in drag values, which has a considerable effect on the $C_{L} / C_{D}$ ratio. Although the plots presented thus far have shown the influence of Reynolds number on the airfoil performance using a numerical prediction tool, XFOIL, these trends have been experimentally confirmed in Ref. [28].

As shown in Figure 2.6, the lift and drag forces acting on a wind turbine airfoil contribute to both torque and thrust forces, which drive the blade in the direction of the plane of rotation and normal to the plane of rotation, respectively. As can be seen, the effective velocity, $V_{\text {eff }}$ consists of the component of oncoming wind velocity, $V_{\infty}$, as well as the component of velocity due to rotation, $V_{\text {rot }}=\omega r$ where $\omega$ is the rate of rotation, and $r$ is the local radius of the blade. This effective velocity is seen by the airfoil at a flow angle, $\phi$, which is defined as the angle made between the $V_{\text {eff }}$ and the plane of rotation. Thus $\phi$ cannot be changed unless either the oncoming wind, or the rotational velocity is altered. The flow angle can be calculated as the 


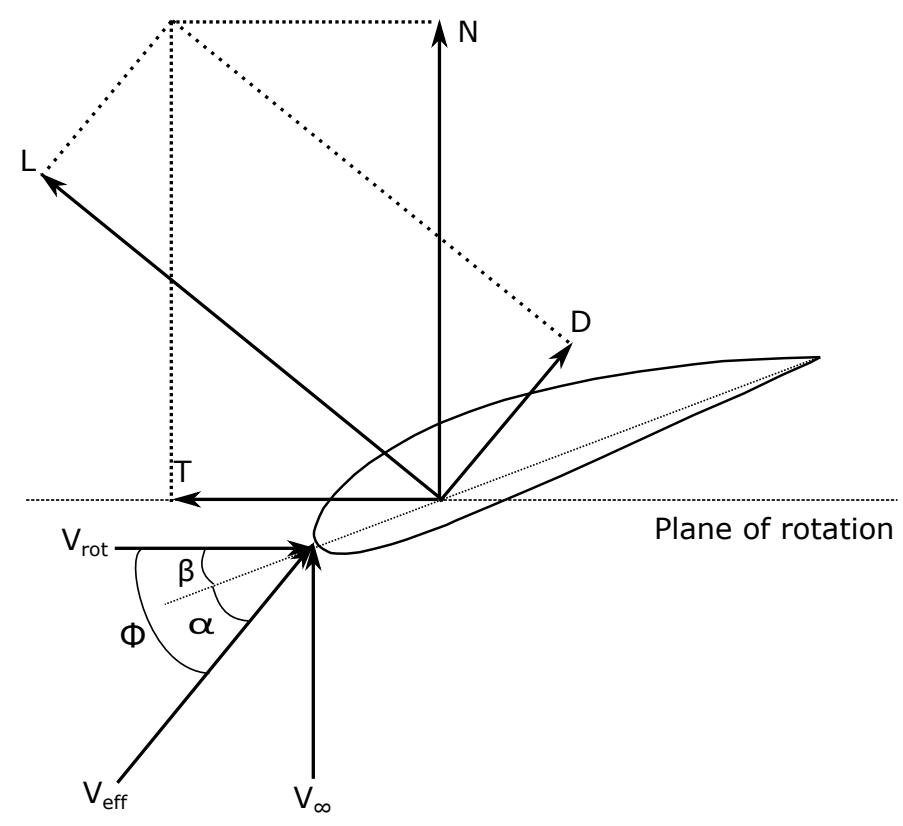

Figure 2.6: The aerodynamic loads acting on a wind turbine airfoil

combination of the angle of attack, $\alpha$, and the blade twist, $\beta$, as seen in Equation 2.5. Alternatively, Equation 2.5 can be re-arranged such that $\alpha=\phi-\beta$. The lift and drag forces acting on the airfoil can be projected into the torque, $T$, and thrust, $N$, directions as a function of the flow angle as described in Equations 2.5-2.7.

$$
\begin{gathered}
\phi=\alpha+\beta \\
T=L \sin \phi-D \cos \phi \\
N=L \cos \phi+D \sin \phi
\end{gathered}
$$

It should be noted that the coefficient of torque, $C_{T}$, and coefficient of thrust, $C_{N}$, can be evaluated by simply replacing $L$ and $D$ in Equations 2.6 and 2.7 by $C_{L}$ and $C_{D}$, respectively. Since the rotational component of velocity is a function of the blade radius, for a given oncoming wind speed the flow angle becomes increasingly large as the radius decreases. In order to ensure that the airfoil operates at its ideal angle of 
attack, the blade twist must be adjusted accordingly; hence the reason why the inner region of the blade has high levels of twist relative to the outer regions.

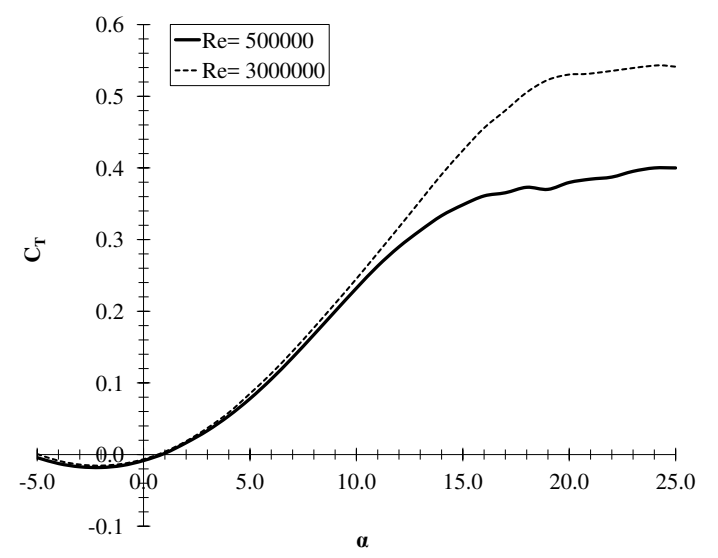

(a) Coefficient of torque

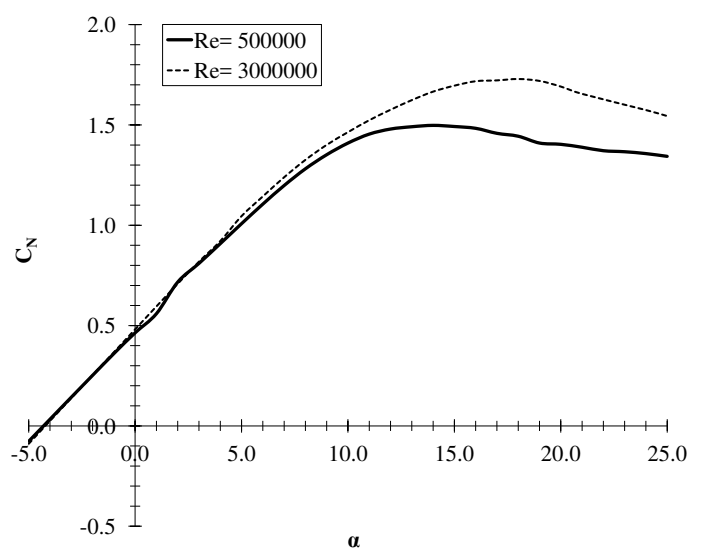

(b) Coefficient of thrust

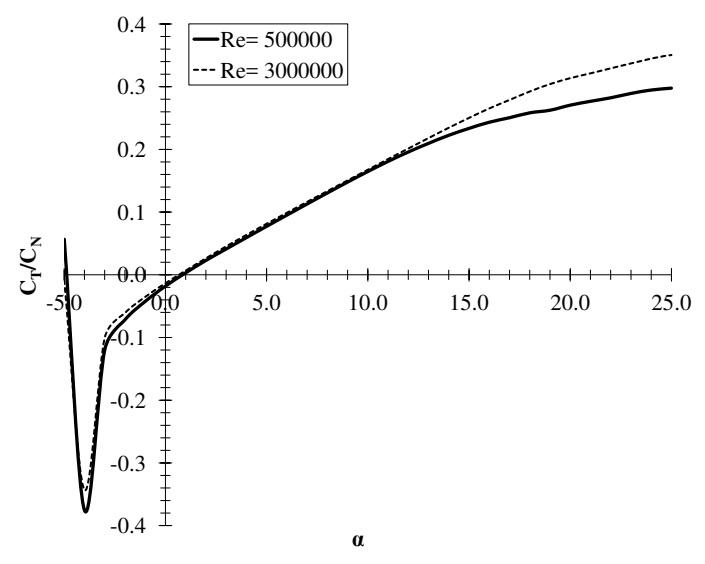

(c) Torque-to-thrust ratio

Figure 2.7: NACA4415 airfoil torque and thrust characteristics

The torque and thrust characteristics of the NACA4415 airfoil evaluated earlier are presented in Figure 2.7(a)-(c). For all plots, the local twist angle of the blade, $\beta$, is assumed to be zero degrees; the effect of changing the twist angle will be analyzed shortly. Figures 2.7(a) and (b) show that both the torque and thrust generally increase with the angle of attack. However, the torque values are observed to become positive 
only when the angle of attack is approximately one degree. When both $\beta=0^{\circ}$ and $\alpha=0^{\circ}$, the lift vector is acting solely in the direction of thrust, therefore the only force contributing to torque is the drag force, which is acting in the direction opposite to the rotation (i.e. negative torque). A possible measure of an airfoil's efficiency on a wind turbine is its torque-to-thrust ratio, which is a measure of the production forces created by the airfoil, torque, relative to the losses, thrust, as shown in Figure 2.7(c).

The discussion thus far has assumed that the blade twist, $\beta$, is zero, however, in reality this is not the case; it is adjusted such that the airfoil operates at its optimal point- the following example will demonstrate this. Assume a flow angle, $\phi$, of $25^{\circ}$ which corresponds to the flow velocities at approximately $25 \%$ of the blade span, assuming an oncoming wind velocity of $10 \mathrm{~m} / \mathrm{s}$, a blade length of $55 \mathrm{~m}$ and a rotational speed of $15 \mathrm{rpm}$. As shown in Figure 2.8(a), adjusting the twist between $\beta=0,10,20^{\circ}$, the $C_{T} / C_{N}$ performance at the corresponding $\alpha=\phi-\beta$ can be determined. From this figure, for a NACA4415 airfoil, it can be seen that as the twist is increased, the corresponding $C_{T} / C_{N}$ also increases up until a certain point. If smaller increments of $\beta$ were used, it would be found that a maximum $C_{T} / C_{N}$ occurs approximately when the twist is set to $19^{\circ}$. 


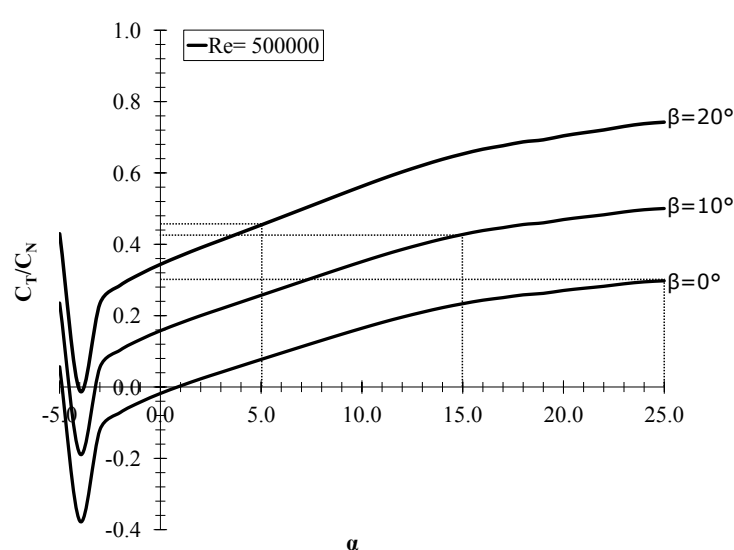

(a) Flow angle $=25^{\circ}$

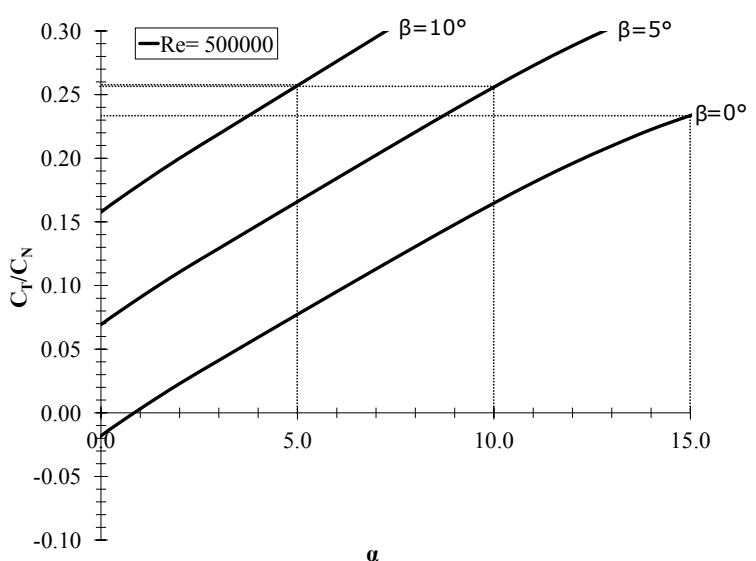

(b) Flow angle $=15^{\circ}$

Figure 2.8: The effect of changing the blade twist, $\beta$, on the airfoil's wind turbine operational efficiency at the $30 \%$ and $70 \%$ span locations

In Figure 2.8(b) where it is assumed that the flow angle is $15^{\circ}$, which corresponds to approximately $70 \%$ of the blade span, the optimal twist angle lies between $5^{\circ}$ and $10^{\circ}$ (approximately $6^{\circ}$ ), not $19^{\circ}$ as was the case for the $25 \%$ blade span location. It should be noted that the scales of the axes in this plot, as well as the values of $\beta$, have been modified such that the region of interest in the plot is clearly shown. Interestingly, in both the inner and outer blade region cases, the optimal blade twist is found to correspond the airfoil's ideal $C_{L} / C_{D}$ angle of attack. In other words, the ideal blade twist seems to correspond to when an angle of attack of about $6^{\circ}$ is achieved, which is where the maximum $C_{L} / C_{D}$ was found for this particular airfoil.

This indicates that $C_{L} / C_{D}$ can be considered an appropriate measure of an airfoil's performance, even for wind turbine airfoils. Recalling Equations 2.6 and 2.7, they can be re-written as seen below, where the torque and thrust force is seen to be not only a function of the lift force, but also the lift-to-drag ratio.

$$
C_{T}=C_{L}\left(\sin \phi-\frac{\cos \phi}{C_{L} / C_{D}}\right)
$$




$$
C_{N}=C_{L}\left(\cos \phi+\frac{\sin \phi}{C_{L} / C_{D}}\right)
$$

Since a high $C_{L} / C_{D}$ value is associated with a smaller loss in torque, it is desirable to possess as large of a value of $C_{L} / C_{D}$ as possible. Furthermore, a high $C_{L}$ will increase the torque force which will increase the power production, however, will also come with an undesirable increase in thrust loading. That being said, a high $C_{L}$ allows for a reduction in chord lengths thereby reducing the solidity of the rotor and in turn, decreasing the loads on the rotor in a parked condition. Depending on the design requirements of the wind turbine, an appropriate $C_{L}$ and a high $C_{L} / C_{D}$ must be incorporated into the airfoil design. The trend of increasing rotor diameters which allow for more energy capture, comes with a trend of more slender rotor blades which reduce the parked loads, but require higher $C_{L}$ values [2].

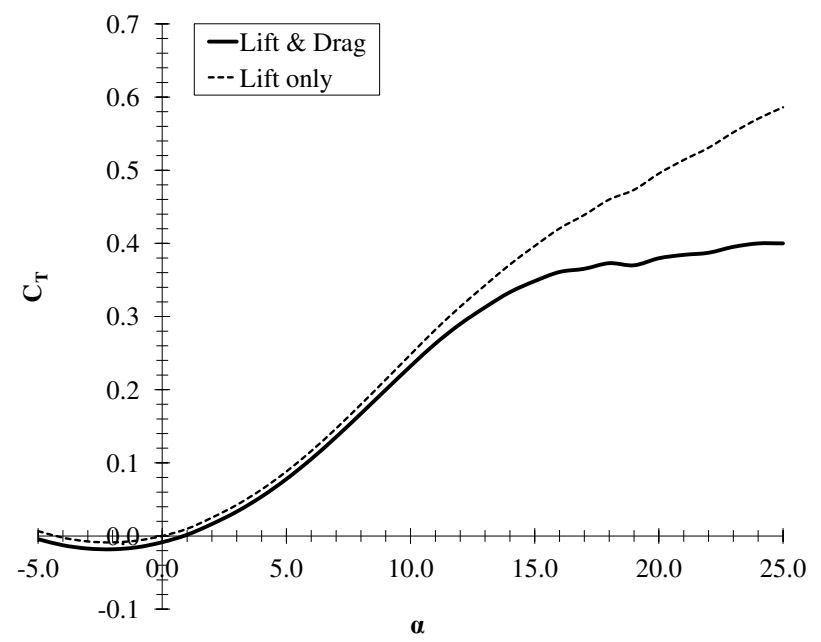

Figure 2.9: The coefficient of torque for an airfoil when drag effects are included and not included

Figure 2.9 shows the torque production of an airfoil with $\beta=0^{\circ}$ including both the lift and drag effects, as well as solely the lift. From this plot, it is apparent 
that at low angles of attack, up to about $13^{\circ}$, the effects of drag have little effect on the torque production of the airfoil. This angle of $13^{\circ}$ corresponds to the airfoil's stall angle at which point the drag rapidly increases. Therefore, the torque force is dominated by lift, and drag can at times be considered to have little impact- this makes high lift a desirable feature for the inboard region of the blade where velocities are low. However, at the outer regions of the blade where the flow velocities are higher and aerodynamic noise and loading are of greater concern, drag should be taken into consideration [29].

Although an airfoil has a particular operating point at which it operates most efficiently, the unpredictable, and constantly varying nature of the oncoming wind makes it difficult to ensure that the airfoil is operating under its ideal condition [30]. For this reason, wind turbine airfoils must operate well over a range of angles of attack; generally, the design angle of attack is chosen to be a few degrees below the stall angle so that a rapid increase in angle of attack does not lead to stall [31] and typically corresponds with maximum $C_{L} / C_{D}$.

\section{$2.2 \quad$ Flatback Airfoils}

The term flatback airfoil is primarily used in the wind turbine industry to describe airfoils with a thick, blunt, or flat trailing-edge. The effect of cut-off, truncated symmetrical airfoils was first studied by Hoerner [32] in 1950 and it was concluded that by thickening the trailing-edge of $25-35 \%$ thick airfoils, often found on the inner regions of propellers, an increase in lift can be achieved. This increase in lift comes as a result of a portion of the pressure recovery of the airfoil occurring in the wake of the airfoil, rather than at the trailing-edge. In other words, since the upper and lower 
surfaces will not have equal pressures at the trailing-edge, overall higher pressure coefficients are found over the airfoil surface. Furthermore, a less-steep adverse pressure gradient is formed which leads to less flow separation and in turn a higher maximum $C_{L}$. The penalty of having a thick trailing-edge was found to be an increase in drag, caused by a rise in profile drag relative to that of a thin trailing-edge airfoil [33]. However, increases in the aerodynamic efficiency, $C_{L} / C_{D}$, often arise particularly for thick airfoils, thanks to the larger increase in lift, relative to the drag.

A study performed on a thinner but similar, NACA 0012 airfoil, in which various amounts of the trailing-edge was removed found similar results, however, the increase in lift was less pronounced in comparison to the increase in drag [34]. For all cases, the drag coefficient for a given lift value was greater for the truncated airfoil than the sharp trailing-edge, unmodified NACA 0012. Therefore, the thickening of the trailing-edge is more favorable for thick airfoils where it helps reduce the otherwise large adverse pressure gradient, thereby delaying stall and increasing lift. Futhermore, the increase in pressure drag that comes as a result of a thick trailing-edge will be more noticeable for a thin airfoil. In a similar study performed by Ramjee et al. [35], it was noted that the increase in drag associated to thick trailing-edges was mostly apparent at low angles of attack whereas at high angles of attack, the flow separated near the leading-edge, hence the increase in profile drag near the aft portion that arose from the thick trailing-edge had little effect.

Experiments performed by Law and Gregorek [26] several years later examined the effects of truncating a cambered, 21\% thick NACA 64-621 airfoil. After truncation, the relative thickness of the airfoil became $30 \%$ due to a reduction in chord length, and the removal of the aft section of the airfoil, where most camber was present, made for a near-symmetrical airfoil. It was found that the truncated airfoil exhibited a superior maximum lift, a steeper lift slope, as well as improved lift-to-drag performance than 
other airfoils with an equivalent thickness. It was proposed to utilize the truncated NACA 64-621 airfoil in the root section of wind turbine blades where the airfoil must be thick enough to accommodate the large structural spars, yet still feature favorable aerodynamic properties [26].

Experiments were performed by Sato and Sunada [36] at Reynolds numbers below $1 \times 10^{5}$, where for all airfoil models, the chord length was adjusted to account for truncation. Unlike the other experiments previously discussed, this resulted in the same maximum thickness ratio for all airfoils. The beneficial effects of the blunt trailing-edge became evident by causing a delay in the formation of laminar separation bubbles, commonly found at low Reynolds numbers, thereby increasing the lift characteristics of the truncated airfoil in comparison to the sharp trailing-edge airfoil.

Standish and van Dam [29] presented a unique method of designing and analyzing the effect of increasing the trailing-edge thickness of an airfoil, without altering the airfoil's relative thickness or camber which comes as a result of truncation. By symmetrically adding thickness about the camber line to the top and bottom surfaces of an airfoil, and blending this thickness into the original airfoil shape, the trailingedge was increased in thickness independently of the airfoil's maximum thickness or camber. From this study, not only were the beneficial effects of an enlarged trailingedge on a thick airfoil such as an increase in maximum lift, lift slope, and lift-to-drag ratio realized, but they were isolated to come as a result of solely an increase in trailing-edge thickness. Furthermore, it was found that the flatback airfoils demonstrated superior insensitivity to leading-edge roughness by decreasing the adverse pressure gradient such that the turbulent flow remained attached for longer. An over-prediction in lift, and discrepancies in stall angle were found for numerical simulations when compared to experimental validation data, and are likely due to the unsteady, and three-dimensional flow originating from the blunt trailing-edge. 
Figure 2.10 shows an example of an unmodified typical wind turbine airfoil (left), as well as a truncated (middle) and thickened trailing-edge version (right). From this figure, it is clear that by truncating an airfoil, both the camber and the thickness relative to the chord, which is now shortened, changes. In order to maintain a given amount of camber and maximum relative thickness, thickness can be distributed evenly about the trailing-edge as seen in Figure 2.10(c).

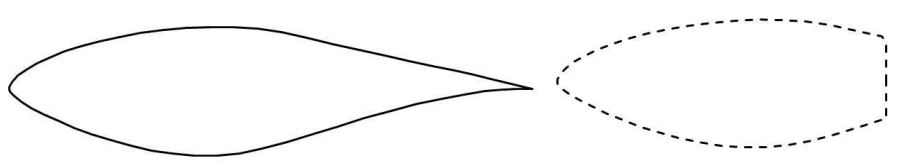

(a) Unmodified

(b) Truncated

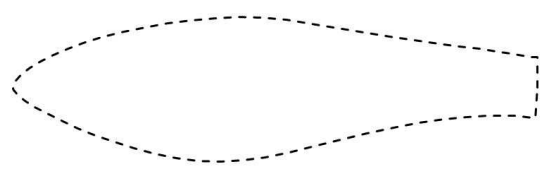

(c) Thick trailing-edge

Figure 2.10: A wind turbine airfoil (left) that has been transformed into a flatback airfoil by truncation (middle) and thickening of the trailing-edge (right)

Experiments performed at $R e=333000$ and $R e=666000$ by Post [37], as well as Baker $[38,39]$ for flatback airfoils created by symmetrically adding trailing-edge thickness both indicate that the stall angle, as well as the lift characteristics are increased with larger trailing-edges. Three different trailing-edge thicknesses for the $35 \%$ thick flatback airfoil tested by Baker et al. were studied and it was found that for the largest trailing-edge thickness of $17.5 \%$, a decrease in $C_{L} / C_{D}$ was obtained, whereas for a moderate trailing-edge thickness of $8.75 \%$, an increase in $C_{L} / C_{D}$ was realized, particularly at large angles of attack due to the delay in stall. Under tripped conditions, which are intended to mimic a dirty leading-edge, Baker established that both the $8.75 \%$ and $17.5 \%$ thick trailing-edge flatback airfoils outperformed the thin trailing-edge airfoil in terms of $C_{L} / C_{D}$, highlighting their good insensitivity to leadingedge roughness.

In an attempt to lessen the negative drag and noise effects that come as a result of a blunt trailing-edge, aerodynamic add-ons and modifications such as Gurney flaps, 
splitter plates, cavities, wavy trailing-edges, and suction and blowing techniques (see Figure 2.11(a)-(d)) were experimentally and numerically studied [39-43]. The use of Gurney flaps, a small tab extending downwards from the lower edge of the trailingedge, had little effect on drag reduction, whereas a plate extending outwards normal to the flat trailing-edge, referred to as a splitter plate, was found to reduce base drag by up to $50 \%$. The splitter plate obtained its effectiveness by interrupting the vortices being shed off the trailing-edge, thereby reducing the base drag. Cavities, formed by implementing two splitter plates attached to the flat trailing-edge, reduced drag by up to $25 \%$. The use of a wavy trailing-edge, that is a trailing-edge that varies in thickness sinusoidally along its span, showed its potential by reducing both drag and aero-acoustic noise on flatback airfoils by creating several smaller vortices which prevent the formation of the typical large vortices that form at the trailing-edge of flatback airfoils. Seele et al. [43] found that the implementation of boundary layer suction and blowing techniques generally helped to delay separation on airfoils with blunt trailing-edges.

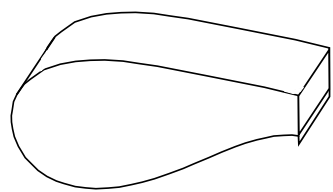

(a)

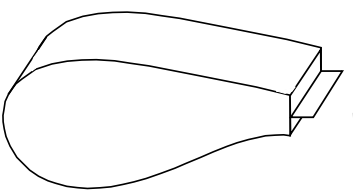

(b)

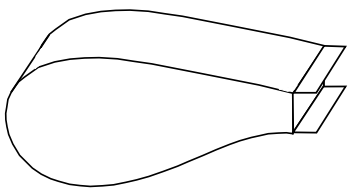

(c)

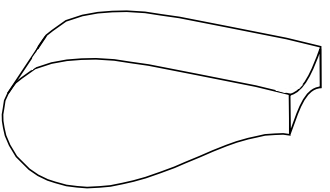

(d)

Figure 2.11: Trailing-edge modifications used in an attempt to alleviate drag and noise effects; (a) Gurney flap, (b) Splitter plate, (c) Cavity, (d) Wavy trailingedge

Several other experimental studies [44-46] of flatback airfoils were performed with similar findings of increased drag and lift, however, a study performed on a 2.3MW wind turbine [7] provided insight into flatback airfoil performance under more realistic, full-scale conditions. Although 2D, constant cross-section wind tunnel tests provide valuable information about an airfoil, the flow over a real wind turbine is $3 \mathrm{D}$ 
in nature. At the inboard regions of the blade, where flatback airfoils are typically found, the flow over the blade has a large component not only in the chordwise direction, but also in the spanwise direction due to the presence of a radial pressure gradient coming as a result of centrifugal and Coriolis effects [47-50]. This additional component of flow, provides energy to the boundary layer, thereby delaying separation and increasing lift performance. This rotational augmentation was found to exist on full-scale rotors, with similar trends for flatback airfoils as sharp trailing-edge airfoils. Relative to stationary, 2D wind tunnel experiments, the lift forces acting on the full-scale, rotating flatback airfoils were at times 2-3 times higher proving that the presence of flatback airfoils did not impede the beneficial 3D flow. The high lift characteristics of flatback airfoils, combined with favorable rotational augmentation effects, confirm the beneficial aerodynamic outcome that flatback airfoils provide to wind turbine blade designs.

Computational Fluid Dynamics (CFD) techniques offer an inexpensive, and potentially time-saving, method of characterizing airfoils. Stone et al. [51] performed CFD analysis on two flatback airfoils using a variety of different turbulence models in conjunction with Navier-Stokes based solvers. Although CFD was able to simulate the lift and drag characteristics quite well while the flow was still attached, larger discrepancies were found for separated flow when compared to experimental results; CFD showed rather gentle stall, whereas experimental results exhibited more sudden stall. CFD performed by van Dam found that the use of standard wind tunnel corrections which account for solid and wake blockage were found to be acceptable for use with flatback airfoils, but the unsteady flow originating from the blunt trailing-edge caused some discrepancies at certain high angles of attack [38].

Using CFD, Papadakis et al. [52] simulated traditional flatback airfoil designs, as well as an airfoil with a rounded trailing-edge. Difficulty in achieving an accurate 
stall angle was obtained, however, for attached flow, CFD results agreed quite well with experimental data. Smaller fluctuations in the forces acting on the rounded trailing-edge airfoil were obtained due to a reduction in the magnitude and frequency of vortex shedding. In a study performed by Prospathopoulos et al. [53] at high Reynolds and moderate Mach numbers, the basic flow characteristics of flatback airfoils at low angles of attack were achieved. However, as the thickness of airfoils was increased, the complexity and frequency of the unsteady wake and vortices required more computational power, and therefore time, in order to accurately simulate and did not show such good agreement.

Several other studies which make use of CFD techniques [11,54-59] have been performed, including some which make use of lower fidelity numerical methods such as XFOIL [25]. The workings of XFOIL will be discussed in further detail in subsequent chapters, however, by making use of a 2D viscid-inviscid panel-method, very fast computations can be obtained with still a rather high level of accuracy. That being said, XFOIL predictions generally tend to over-predict maximum and post-stall lift $[60,61]$, and under-predict drag [60,62], particularly for airfoils with high thickness and blunt trailing-edges. For this reason, experimental tests must be performed. As was performed by Muñoz et al. [59], it is possible to empirically adjust XFOIL results using experimental data to better simulate flatback airfoils.

Asides from numerous aerodynamic advantages, flatback airfoils also offer structural advantages such as a smooth transition in shape from cylindrical sections to a sharp trailing-edge airfoils, as well as the advantage of having a higher cross-sectional area and sectional moment of inertia for a given airfoil thickness. The sectional moment of inertia of an object is a measure of its resistance to bending about an axis. For long slender wind turbine blades, the majority of the bending occurs in the flapwise direction, normal to the plane of rotation, therefore a high sectional moment 
of inertia about the chord is essential to resisting deformation in this direction. By having a larger amount of area spread out at a distance from the chord line, the stresses experienced by the loading on the blade are redistributed, making them less intense. Flatback airfoils inherently add thickness to the trailing-edge region of the airfoil thereby increasing the sectional moment of inertia, and in turn offering better resistance to flapwise bending. [63]

Thanks to their attractive aerodynamic and structural properties, flatback airfoils are used in the inboard regions of wind turbine blades where thick airfoils are found. Although manufacturers have already implemented them in their rotor blade designs, little public information is available on their performance at a full-scale level, with the exception of the aforementioned Siemens 2.3-MW study [7] for which flatback airfoils proved many of their aerodynamic advantages. However, the Blade System Design Study (BSDS) performed by Sandia National Laboratories sought out to make improvements to the aerodynamics, structures and manufacture of utility scale wind turbine blades. To reduce costs and resources, this study made use of a customdesigned $9 \mathrm{~m}$ blade in order to validate the aerodynamic and structural concepts. It was found that when flatback airfoils were used, the blade tolerated loads up to $310 \%$ of the design load, and tip-deflections more than double that of the sharp trailingedge blades [64]; the increased strength allows for less material to be used in the manufacture of the blades. The increase in lift relative to sharp trailing-edge airfoils has potential for more slender blades to be produced thereby reducing loading under stand-still, parked rotor conditions, and allowing for easier transport of the blades due to reduced maximum chord lengths [65].

Using a grid-less CFD technique called Blade Element Momentum (BEM) theory, a $50 \mathrm{~m}$ blade based on the $9 \mathrm{~m}$ BSDS blade was simulated [66]. The inclusion of flatback airfoils in the design proved to be an effective means of improving the 
structural properties of the blade, without sacrificing aerodynamic performance. The flatback airfoils performed particularly well under soiled conditions outperforming their sharp trailing-edge counterparts. Furthermore, the noise concerns associated with the blunt trailing-edge of flatback airfoils were discounted since trailing-edge noise intensity scales with $V^{5}$ [67], and velocities at the inboard regions, where flatback airfoils are present, are low.

Projections from today's in-production blades show that the mass of the blade increases with a scaling factor of 3.0 in relation to the blade radius, however, the inclusion of flatback airfoils into a hypothetical $100 \mathrm{~m}$ blade design showed that a scaling factor of 2.2 could be achieved meaning that lighter than typical designs are possible [68]. Furthermore, the Annual Energy Production (AEP) of the rotor using flatback airfoils was maintained at the same level as the rotor with sharp trailing-edge airfoils. Interestingly, in a separate study, a preliminary aero-structural optimization of a hypothetical wind turbine resulted in the natural emergence of flatback airfoils in the design and an increased AEP, however, an increase in blade mass was also realized [69].

\subsection{Design of Wind Turbine Airfoils}

As the first link in the conversion of the wind's kinetic energy into mechanical energy, the performance of an airfoil must be maximized under its intended operating conditions. Although several airfoils used in the aerospace industry have been perfected over the years, due to vastly different operating conditions and structural requirements, they were found to be unsuitable [70]. Early wind turbine airfoil designs were based around rather low Reynolds numbers $\left(R e<3.0 \times 10^{6}\right)$ due to the small rotor blade radii. The design criteria involved maximizing the lift-to-drag ratio of the 
airfoils, and ensuring that the airfoil was rather insensitive to leading-edge contamination caused by insects, dirt or erosion, while maintaining or improving structural integrity. The Eppler airfoil design code, based upon a computationally inexpensive panel-method [71], was used to design a set of airfoils ranging from 11-26\% in thickness as seen in Figure 2.12. The thicker airfoils are intended for the inboard region of the blade where velocities are low and structural requirements are high, whereas the thinner airfoils provide an excellent lift-to-drag ratio at the outer regions of the blade where velocities are highest [70]. It can be seen that as thickness increases, the leading-edge radius also increases, and the S-shaped lower surface becomes more pronounced. It was determined that by using custom designed wind turbine airfoils, a 10-15\% improvement in AEP could be realized compared to conventional aircraft specific airfoils [72]. It should be noted that the SERI designation used to name the airfoil family was later changed to National Renewable Energy Laboratory (NREL).

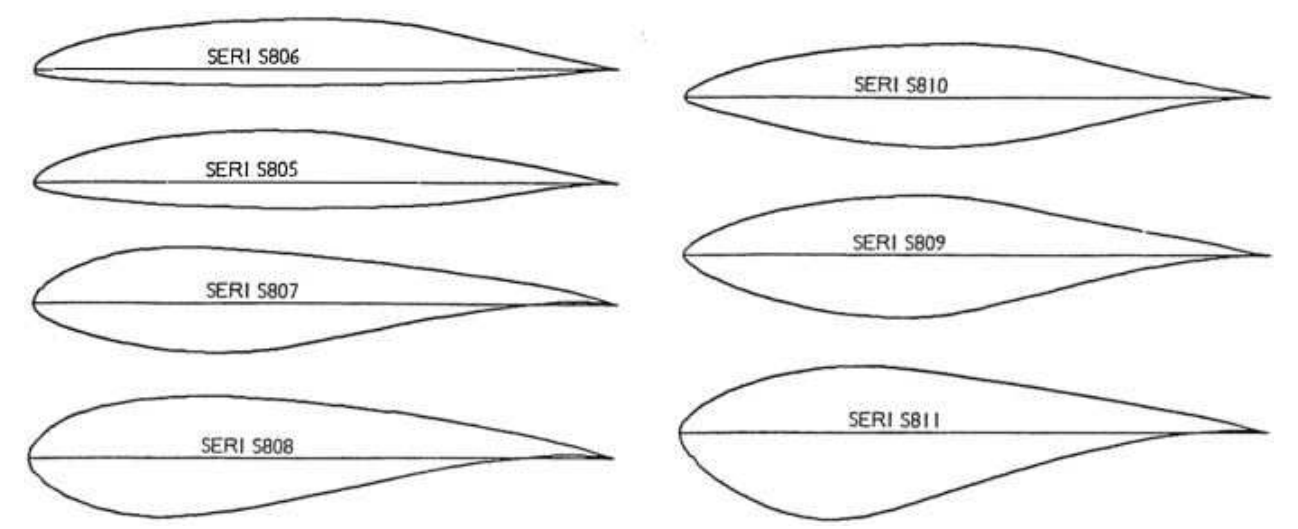

Figure 2.12: Family of wind turbine specific airfoils designed by NREL [70]

A few years later, the Aeronautical Research Institute of Sweden (FFA) designed their own family of wind turbine airfoils [73] for blades up to $45 \mathrm{~m}$ long, meaning that $R e>2.0 \times 10^{6}$ in all cases. At the outer regions of the blade, where the geometric twists are lowest, lift-to-drag ratio was of utmost importance since the airfoil is almost 
in-line with the plane of rotation, whereas at inboard regions, structural concerns were paramount. All airfoils were designed by prescribing a velocity (or pressure) distribution over the airfoil, and then using XFOIL's inverse design methodology to determine the shape. Once again, a primary design requirement was that the airfoils perform well under dirty conditions. This was accomplished in the design by ensuring that good performance was maintained when transition to turbulence was imposed near the leading-edge at the $1 \%$ and $10 \%$ chord locations of the upper and lower surfaces, respectively. From this, three families of airfoils, the first having high lift for low-wind sites (FFA-W1-XXX, 13-27\% thick), the second having lower lift for high-wind sites (FFA-W2-XXX, 13-27\% thick), and the third having thicker airfoils (FFA-W3-XXX, 19-36\% thick) were established; the first and third families are shown in Figure 2.13. As can be seen the thicker, FFA-W3-XXX airfoils have more aft-camber with the trailing-edge pointing slightly more downwards than the FFA-W1-XXX airfoils which provides good lift-to-drag, despite the high thickness. The emergence of slightly flatback airfoils can be seen in the thickest of the FFA-W3XXX airfoils. Lastly, it can be seen that for a given airfoil family, the change in shape with respect to thickness is very gradual allowing for a smooth transition in shape along the span of the blade, thereby facilitating the manufacturing and structural compatibility.

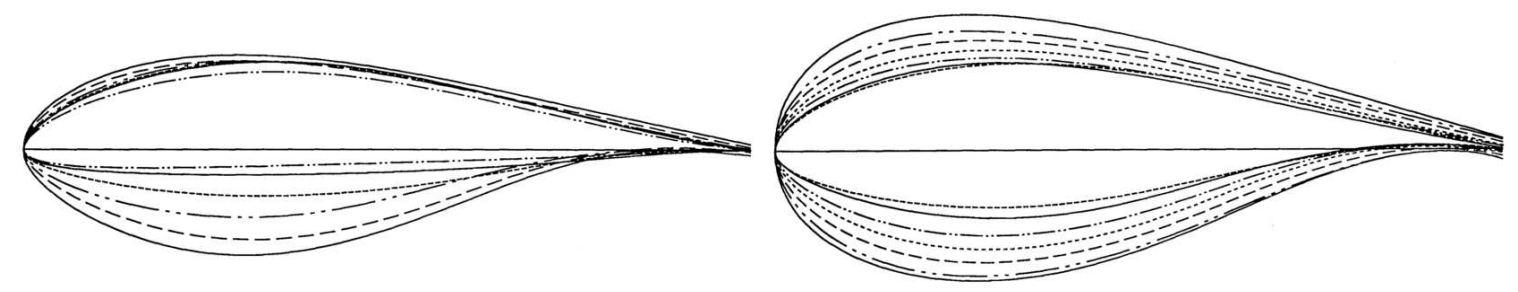

Figure 2.13: Family of wind turbine specific FFA-W1-XXX (left) and FFA-W3XXX (right) airfoils [73]

Research groups at the Delft University of Technology (DUT) began their own 
design of wind turbine airfoils soon thereafter. An airfoil family, seen in Figure 2.14, ranging in thickness from 15-40\% was created over the span of several years and have since been used successfully on a variety of wind turbines. XFOIL, and later RFOIL, a modified version of XFOIL which accounts for rotational augmentation, was used to design airfoils with high lift-to-drag, good insensitivity to roughness, consistent location of maximum thickness, and good structural capabilities [62,74]. It was mentioned, however, that for inboard sections, high lift may be the design driver rather than high lift-to-drag ratio [75]. Thickness is primarily added on the pressure surface making flow separation on the suction surface less likely to occur by maintaining reasonable adverse pressure gradients- particularly under soiled conditions. Increasing amounts of camber are added to the aft portion of the airfoil in order to achieve high levels of lift, while maintaining acceptable drag.

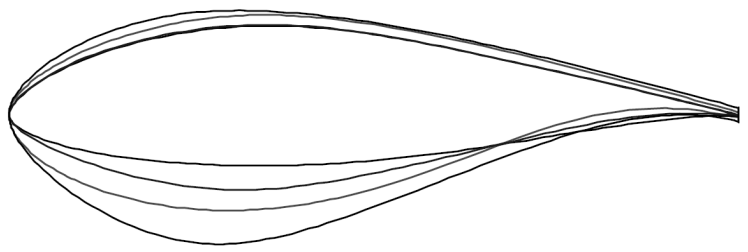

Figure 2.14: Family of wind turbine specific DUT airfoils [75]

Making use of optimization techniques coupled with XFOIL, the Riso National Laboratory in Denmark designed four generations worth of wind turbine airfoil families designed for the ever-increasing lengths of rotor blades [76]. The initial Riso-A1 and Riso-P series of airfoils were designed for stall and pitch regulated machines, respectively, and in both cases, were optimized using $C_{L} / C_{D}$ as the driving aerodynamic evaluator.

The increased chord, and lengths of modern blades, required a new, improved generation of airfoils which operate at higher Reynolds numbers. Therefore, for the 
Riso-B1 airfoils, $R e=6.0 \times 10^{6}$ was used as the operational condition in the optimization. Rather than the traditional method of designing for maximum lift-to-drag, the Riso-B1 airfoils were designed for maximum coefficient of torque, $C_{T}$, the driving force of blade rotation. This design choice put more emphasis on insensitivity to leading-edge roughness, rather than a high lift-to-drag ratio that comes merely as a result of a low drag coefficient. High lift must be retained between clean and soiled conditions meaning that transition to turbulence near maximum lift must naturally occur close to the leading-edge on the suction surface [77]. That way, any roughness caused by erosion, dirt, or insects, which will cause an increase in the boundary layer thickness, will not significantly alter the lift characteristics of the airfoil due to premature separation. Although only the airfoils with 15-36\% thickness are shown in Figure 2.15, airfoils up to $53 \%$ thickness were designed to be used in very inner regions of the blade. Recently, the Riso-C2 airfoils were created with many of the same design features as the Riso-B1 airfoils, however, more emphasis was put on roughness insensitivity, geometric compatibility and stiffness about the chordline. Furthermore, the Riso-C2 airfoils were designed using $C_{L} / C_{D}$ rather than $C_{T}$ as the aerodynamic evaluator, with no clear explanation as to why the aerodynamic evaluator was switched back to $C_{L} / C_{D}$, other than possibly for convenience (inferred by the present author).

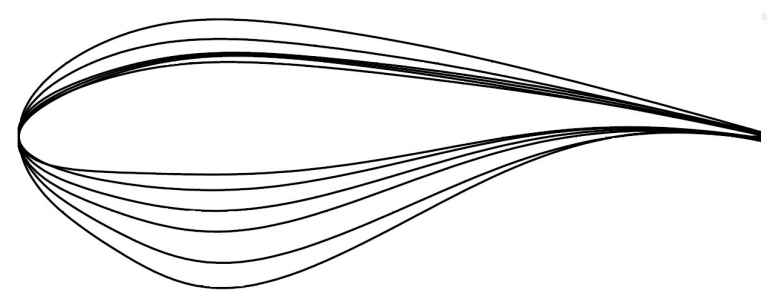

Figure 2.15: Family of wind turbine specific Riso-B1 airfoils [78]

Based on the designs conducted by these major research groups, the airfoil thickness range has been steadily increasing in order to accommodate the larger loads 
introduced due to increased blade lengths of today's wind turbines. They all make use of fast computational codes such as XFOIL during the design phase, after which they experimentally test a select few airfoils in the wind tunnel. Consistently, XFOIL has been found to slightly over-predict lift and under-predict drag, particularly in the stall and post-stall region, showing the need for experimental verification of results before introducing the airfoils into production.

More recent design studies have began to allow freedom in the trailing-edge thickness, particularly when designing for the inboard airfoils of very large blades. Figure 2.16 illustrates several examples of flatback airfoil designs available in the literature $[31,46,59,79-81]$. Depending on the thickness, operating conditions, and desired airfoil characteristics, it can be seen that a variety of shapes will be realized. However, what all airfoils have in common is a pressure surface with the characteristic S-shape, and a trailing-edge that points slightly downwards. For the most part, the airfoils all exhibit a more shallow suction surface compared to the pressure surface which aids to reduce the upper surface velocities thereby preventing premature flow separation under clean and soiled conditions. Furthermore, some of the studies use the sectional moment of inertia, $I_{X X}$, to evaluate the structural performance of the airfoil, however a particular study has used more precise methods where the composite lay-up, and location and size of the structural spar are considered [82].
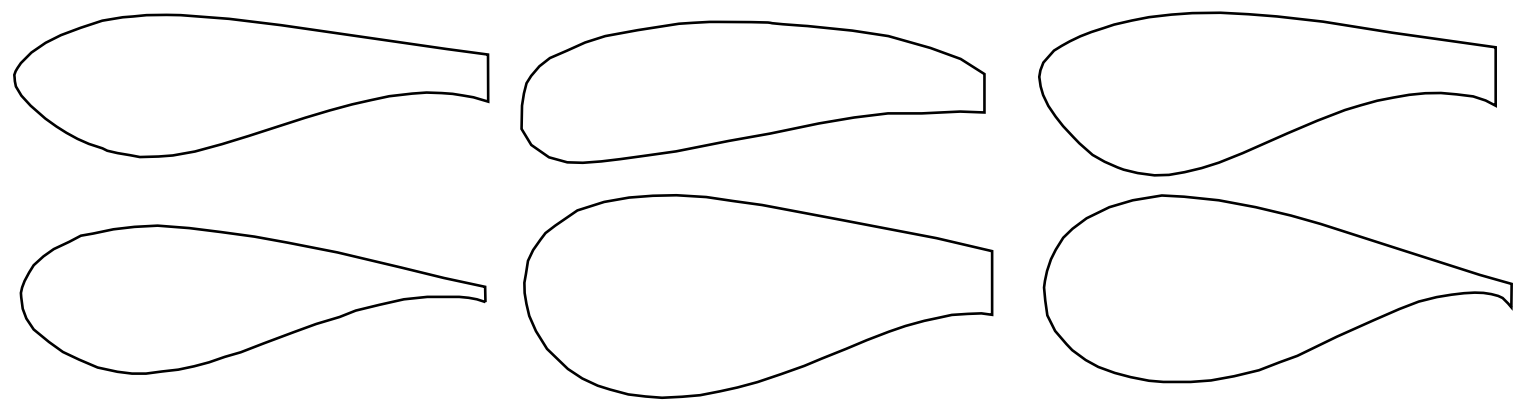

Figure 2.16: Various examples of flatback airfoil designs (adapted from $[31,46,59$, 79-81]) 
From this review of existing airfoil designs, there are many general consensuses regarding desirable wind turbine airfoil characteristics. Table 2.1 shows a summary of desirable wind turbine airfoil characteristics, with thickness, geometric compatibility, structural demands, aerodynamic efficiency, insensitivity to roughness, and noise being the primary design drivers. However, the specifics as to what degree these characteristics are achieved, and how they are achieved during the design phase is not straight-forward. Depending on the design requirements and philosophies, different design constraints and objectives may be necessary for a given aerodynamic evaluator. As shown in the design for the Riso airfoils, $C_{L} / C_{D}$ was most commonly used as the aerodynamic evaluator during the design process, however, $C_{T}$ was also successfully used with similar results. The role and choice (and reasoning) of the aerodynamic evaluator, along with the specific constraints and objectives required for each, are not clear. Systematic research to evaluate how changing the aerodynamic evaluator under a set of constraints and objectives, as well as other parameters, will be performed in this work to obtain a better understanding of the airfoil design process.

Table 2.1: General characteristics desired from wind turbine airfoils (adapted from Ref. [17])

\begin{tabular}{|c||c|c|c|}
\hline \multicolumn{4}{|c|}{ Desirable Airfoil Characteristics } \\
\hline Characteristic & Thick Airfoil & Medium Airfoil & Thin Airfoil \\
\hline Thickness & $>30 \%$ & $21-30 \%$ & $<21 \%$ \\
Structural Demands & +++ & ++ & + \\
Geometric Compatibility & ++ & ++ & ++ \\
Aerodynamic Efficiency & + & ++ & +++ \\
Insensitivity to Roughness & + & ++ & ++ \\
Noise Concerns & & + & +++ \\
\hline
\end{tabular}




\section{Chapter 3}

\section{Numerical Optimization}

In engineering, the goal of optimization is to ensure that a design performs as effectively and efficiently as possible given the design constraints. However, as engineering problems are often multi-disciplinary [83], this optimization process involves tradeoffs between factors such as weight, cost, and efficiency. In the case of wind turbine airfoils, aerodynamic and structural performance are mutually exclusive meaning that an optimal aerodynamic design will be sub-optimal structurally and vice-versa. That is to say that an aerodynamically efficient airfoil is often associated with a thin airfoil, which due to its small cross-sectional area and slender shape, is structurally non-ideal. As wind turbine blades continue to grow in length, the aerodynamic and gravitational loads also increase substantially thereby providing a need for aero-structurally optimized airfoils, particularly in the inboard region of the blade where loading is highest.

Classical optimization schemes typically focus on a single design factor to be optimized. This results in a single design, which for the given conditions and constraints, will be optimally performing. In multi-objective optimization, where more than one objective is to be simultaneously optimized, a range of optimal solutions will be obtained. When these objectives are conflicting, such as aerodynamic and structural performance of an airfoil, a "family" of designs will be established with 
varying degrees of aerodynamic and structural performance, respectively. A variety of algorithms including gradient-based and evolutionary-based methods can be employed for single or multi-objective optimization. Some optimization fundamentals, gradient and evolutionary-based methods, optimization specific to wind turbine airfoils, methods employed in the present study, as well as findings from this study will be described in the subsequent sections.

\subsection{Fundamentals of Numerical Optimization}

From a mathematical standpoint, optimization involves the maximization or minimization of a given function. Most optimization algorithms solve for the minimum, however, the maximum of a given function can be found by taking the minimum of the negative of the same function [84]. The example below will illustrate a few key elements of an optimization problem.

Consider the following example function, which is plotted in Figure 3.1 and will be used to help describe some terminology:

$$
y(x)=x^{4}+4 x^{3}+3 x^{2}-4 x+1 \quad \text { where }-3 \leq x \leq 1
$$

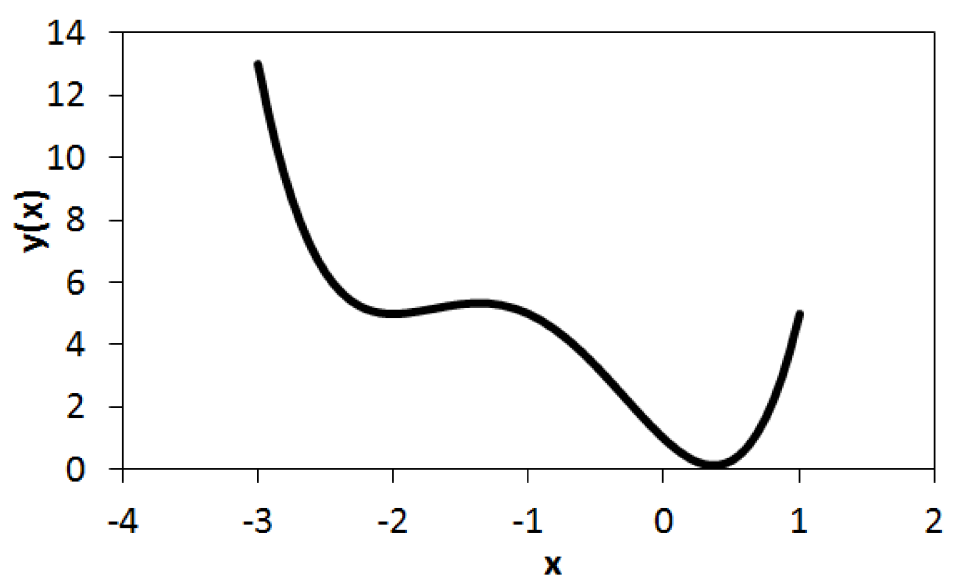

Figure 3.1: Example function 


\subsubsection{Terminology}

Objective Function: Also referred to as the fitness function, the objective function is the function to be maximized or minimized. In this example, $y(x)$ is the objective function to be minimized. If the maximum of a function is sought, this can be obtained by minimizing $-y(x)$.

Variables: The parameters which will change the value of the objective function, in this case represented by $x$.

Bounds: The inequalities or limits imposed on the value of the variables. In this case, values of $x$ must be between -3 and 1, inclusively. Unconstrained optimization will allow the variables to take on any value.

Minima/maxima: The lowest or highest known value of the objective function, respectively. These can be subdivided into local and global minima/maxima which are the lowest/highest value in a given range and the lowest/highest value in the entire domain. In the above example, a local minima exists when $x=-2$ and a global minima exists when $x=0.4$.

Continuous Optimization: Values of the variable are continuous if they can take on any and all real numbers (provided they fall within any constraints). Continuous optimization problems are typically easier to solve mathematically due to the smoothness of the function which allows for more information to be inferred regarding the behaviour of the function at a given point [84].

Discrete Optimization: Values of the variable are drawn from a finite set of numbers which may include exclusively discrete or binary values [84]. An example of discrete optimization would be if one was desired to find the optimal number of cars and buses required to transport a given number of passengers, a negative or non-integer value for the number of cars would be nonsensical. A constraint which imposes that all values must be an integer value, greater than zero could be utilized 
to solve this issue.

Multi-objective optimization: When more than one objective function is to be optimized simultaneously. This is the basis of multi-disciplinary optimization.

Pareto front: When more than one optimal solution exists, as it the case with multi-objective optimization problems, a "family" of best solutions is created. This family, or Pareto front, represents the best solutions found thus far. See Figure 3.2 below.

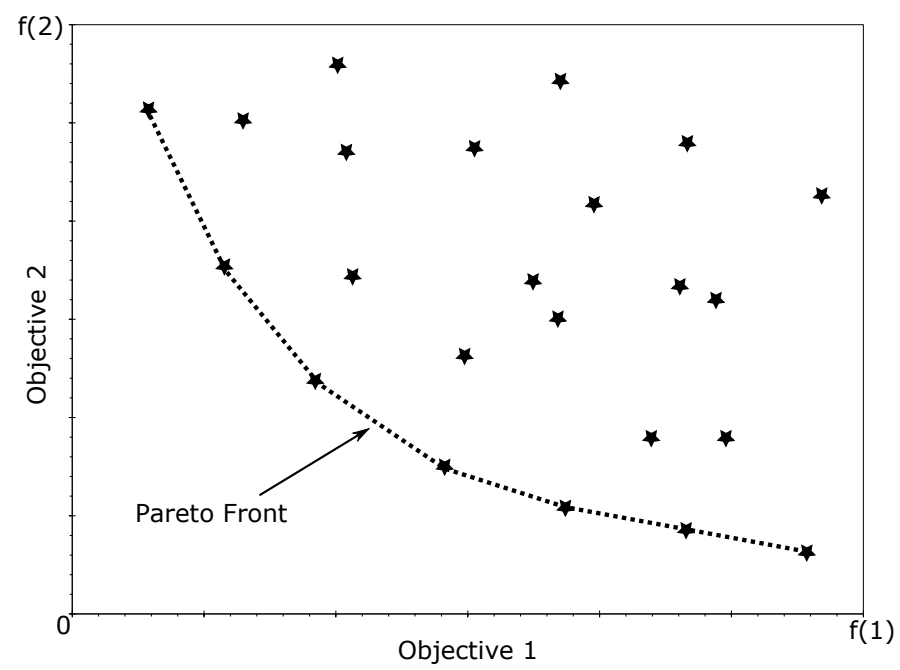

Figure 3.2: Example Pareto front showing the optimal evaluations given two objective functions. Note that since this is a minimization problem, smaller values are considered optimal

\subsubsection{Optimization Techniques}

Although several optimization techniques can be used for multi-objective design, the majority of techniques can be categorized into two main methods: gradient-based optimization and (semi-)stochastic optimization.

Gradient-based optimization makes use of the first and/or second derivative of the fitness function to determine in which direction lies the steepest gradient and in 
turn, minimum of the function [84]. Also known as the steepest descent (or ascent) optimization, this method is analogous to descending into a valley. Naturally, if you would like to get to the shallowest part of a mountain range in the most efficient manner, you would take your first step in the direction of the valley visible to you. From there your next steps will always be in the direction where the gradient of the slope is steepest. This technique will get you to the bottom of the valley quickly, however, you cannot be sure that on the other side of the mountain range, lies another valley at a lower altitude than the one visible from your current position. The effectiveness of this technique is often very dependent on the starting position of the solution, and although this method is very efficient at getting to a minima quickly, it may mistake a local minima with a global minima. This is particularly problematic when several local minima surround the global minima or when there is not one solution to a given problem. Methods which randomly re-direct the direction of descent attempt to alleviate this issue by forcing the solution to explore new directions in which the global minima may lie. Gradient-based techniques often must calculate the Hessian matrix which contains the local curvature (second-order partial derivatives) of the function with respect to several variables. This calculation process can be computationally costly and for certain problems, infeasible [85].

(Semi-)Stochastic optimization involves the assignment of random-value variables within the bounds or constraints of the problem to evaluate the fitness function. By ensuring a large scatter in values of the design variables, it is ensured that a nearglobal minima is achieved. A purely stochastic technique will simply continue to vary the value of the variables randomly until the stopping condition is achieved, however, this is very inefficient. For this reason semi-stochastic, or heuristic, techniques are more popular as they strategically assign values to the variables based on previous results of the fitness function. Evolutionary algorithms, also referred to as genetic 
algorithms, are a popular example of a heuristic optimization technique and will be described in greater detail shortly.

Many of the shortcomings of gradient based methods are the strengths of genetic algorithms. Genetic algorithms are relatively simple to set-up, with design constraints being included either before the evaluation of the design by limiting the values of variables, or after the design by assigning a fitness value of zero if the variables fall outside certain bounds (a penalty function) [86]. Genetic algorithms are very robust and can be easily used for multi-objective problems where more than one optimal solution exists [87]. Furthermore, genetic algorithms should find the nearglobal optimum and have a lower likelihood of getting stuck in local minima. That being said, these advantages come at the cost of increased computational time due to the large design space that must be searched, as well as the slow convergence, particularly near minima [88].

Since this study involves the optimization of flatback airfoils for both the aerodynamic and structural characteristics, which are mutually exclusive, more than one optimal solution will exist. For this reason, an optimization technique which is robust for multi-objective designs, and simple to manipulate when performing various analyses, is required. A genetic algorithm, gamultiobj, from MATLAB was therefore employed for this study [89].

\subsection{Implementation of Optimization for Flatback Airfoils}

A numerical optimization tool used to optimize the shape of flatback airfoils under certain operating conditions was developed. This tool couples an existing airfoil prediction tool, XFOIL, with MATLAB's gamultiobj multi-objective genetic algorithm 
to provide an efficient and robust optimization program. As seen in Figure 3.3, the program can be broken down into several components including XFOIL, MATLAB's gamultiobj, a coupling between the two aforementioned programs, and an airfoil shape generator- all of which will be described below.

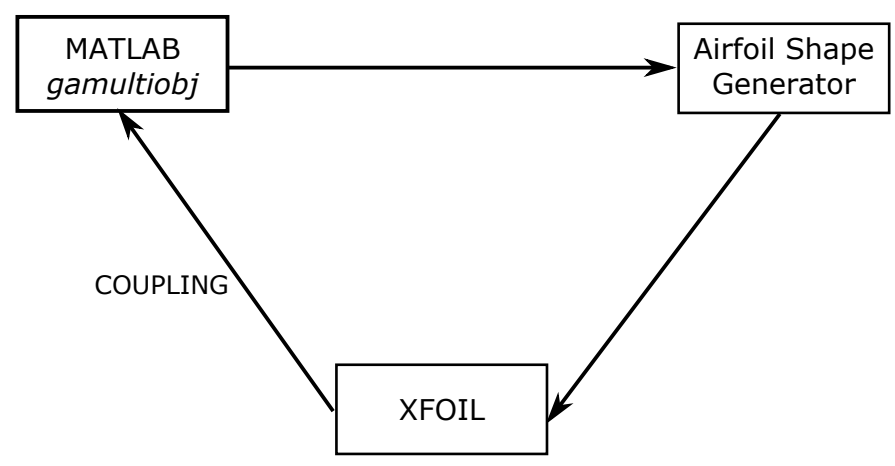

Figure 3.3: Simplistic overview of flatback airfoil optimization software architecture

\subsubsection{MATLAB gamultiobj}

MATLAB's gamultiobj offers a robust optimization tool for solving multi-objective problems where several optimal solutions may exist. The basic working principal of genetic algorithms can be summarized by the flow chart shown in Figure 3.4.

An initial population is created containing a set number of individuals. These individuals can either be randomly generated within the constraints of the problem, or specified by a user-supplied input. Upon evaluating the fitness values for all of the individuals of the population, a rank based on the level of front and crowding operator is assigned to each individual. All of the top performing individuals (Paretooptimal front) are assigned a rank based on their front of 1 . This Pareto-optimal front is temporarily removed from the population, and the new resultant Pareto front is found and assigned a rank of 2. This process is repeated until all individuals have been assigned a rank based on which front they lie on. Within each front, the individuals are 


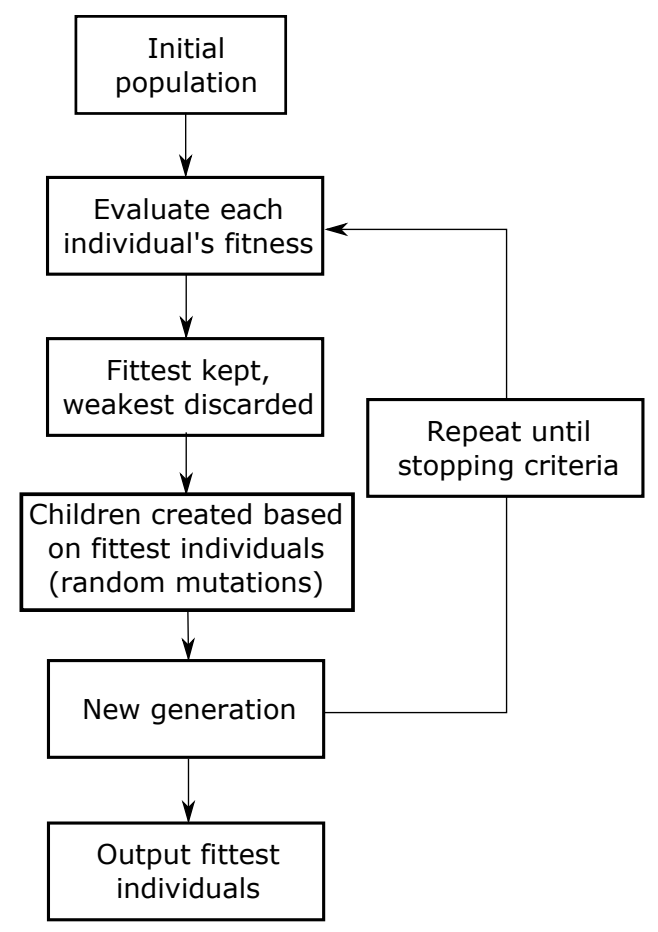

Figure 3.4: Simplified work-flow of a genetic algorithm

further ranked based on their crowding, or distance, from one another. The distance from each individual being evaluated to the neighboring individuals on either side is found and based on this, they are assigned a secondary rank. Individuals which represent the extremities of the front are ranked highest in terms of distance.

A certain fraction (Pareto fraction) of the highest ranked Pareto-optimal front are retained as elite individuals which automatically get passed onto the subsequent generation. The remaining individuals undergo a tournament selection technique in which groups (usually of 4) of individuals are compared based on their ranking, and the best performing are kept as a parents and the weakest are discarded. The two-tier ranking system ensures both optimality, but also diversity by preserving a high level of spread between solutions and maintaining solutions which represent the extremities of the design space. In order to maintain the original population level, several new individuals (children) are created by combining variables (genes) of the 
parents from the generation previous such that the population reaches its designated level. In order to ensure that the algorithm does not get stuck in a local minima, random mutations are occasionally introduced into the children such that all possible variations are evaluated, while still efficiently tending towards better solutions. This process of continuous progression towards the Pareto-optimal solution, one generation after another, continues until the stopping criteria is met.

MATLAB's genetic algorithm makes use of a controlled elitist technique. Based off of a nondominated sorting genetic algorithm (NSGA-II) [90], a controlled elitist technique ensures that the fittest individuals are passed onto subsequent generations, while still allowing select lower fitness individuals to advance such that the population diversity is maintained at a high level. Contrary to an elitist technique, the controlled elitist technique is able to achieve better convergence of the global Paretooptimal front thanks to its ability to maintain a high level of spread of the Pareto optimal solutions [90]. Key details of the NSGA-II technique used in gamultiobj are highlighted below.

\section{Number of Generations}

The number of generations can be specified by the user and may act as a stopping condition for the optimization. A high number of generations will ensure that the optimization has had a sufficient number of iterations to converge to a solution, however, choosing a number too high can result in large increases in computational time, with insignificant improvements in the solution. Therefore, through a convergence analysis, an appropriate number of generations can be selected for the problem at hand. The default value for the number of generations in gamultiobj is $200 \times$ variables [91]. 


\section{Population Size}

The population size specifies how many individuals are to be evaluated in each generation. Too small of a population size typically results in poor exploration of the entire design domain and can lead to convergence in local minima. Large population sizes allow a larger extent of the domain to be explored, however, increase the number of computations, and therefore time of the optimization $[92,93]$. Population size is therefore a parameter that must be determined for a particular optimization problem through a convergence analysis. The default value for the population in gamultiobj for problems with more than 5 variables is 200 [91].

\section{Creation Function}

The creation function specifies how the initial population is generated. The default function creates a random, but uniform, initial population within the constraints of the problem [91]. As mentioned earlier, a user specified initial population matrix can be imposed, however, the initial population may influence the outcome of the optimization.

\section{Selection Function}

The selection function specifies the manner in which parents are chosen from the population of the current generation, for the subsequent generation. For gamultiobj,

a tournament selection function is used in which a number (tournament size) of randomly selected individuals from the population are chosen, and the best of those based on the two-tier rank system described earlier is assigned as a parent; the default tournament size is 4 individuals [91]. This process is repeated until all individuals within the population have undergone tournament selection. 


\section{Crossover Function}

Crossover describes the method in which two individual parents are combined to create a child for the subsequent generation. gamultiobj uses an intermediate crossover technique for multi-objective optimization problems. Equation 3.1 shows the formulation used in the intermediate crossover technique [91].

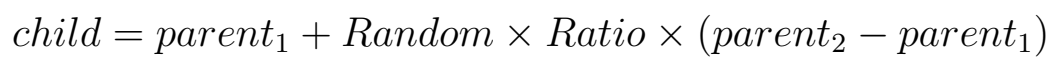

where Random is a randomly generated value between 0 and 1 which acts to randomly combine features (variables/genes) of the two parents. Ratio can be specified by the user if they wish to impose a weighting factor that favors one parent over another; by default this value is set to a value of 1 . Figure 3.5 illustrates a simplistic example where each individual is made up of 4 variables and Equation 3.1 has been applied to each. The numbers in italics represent the randomly generated values. Although intermediate crossover is random in nature, as the solution of the optimization begins to converge, the variables between parents should take on very similar values meaning that the random nature should have little effect on the variable values. The fraction of the population which is created by crossover takes on a value between 0 and 1 and can be user specified. The remaining fraction will be created by means of mutation.

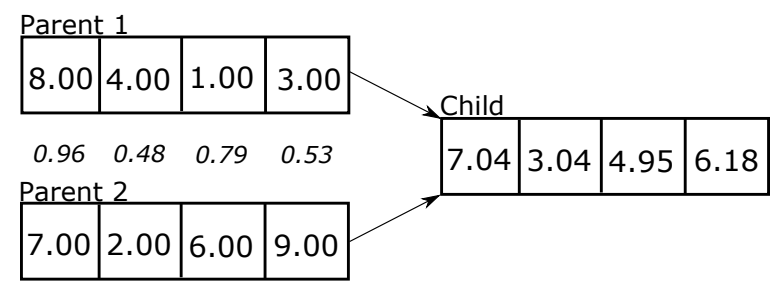

Figure 3.5: An example of the intermediate crossover used in gamultiobj 


\section{Mutation Function}

In order to ensure a diverse population such that all areas of the domain are explored, while avoiding local minima, mutation of children must be included such that one or more of the individual's variables are randomly altered. For gamultiobj, an adaptivefeasible mutation technique is used for which the direction and magnitude of mutation for a particular variable is initially randomly generated. Each mutated variable is then checked to ensure that respects all constraints and bounds. If not, the magnitude, or step size, in the initial direction of mutation is reduced. This process is repeated until the variable falls within the specified bounds and constraints [91,94].

\section{Maximum Stall Generations}

A stopping condition that can be employed for gamultiobj is the maximum number of stall generations. If the average relative change in the best fitness function values do not change by more than $1 \times 10^{-4}$ (default value) over a span of 100 generations (default value), and the optimization has not yet reached the maximum number of generations specified by the user, the optimizer will stop as the solution is no longer improving by a significant amount [91].

Several parameters in gamultiobj can be varied to alter the performance of the algorithm. The primary variables amongst these include population size and number of generations. Each of these two variables were systematically individually changed in order to determine their effect on the convergence of the optimization solution. The results of this study can be found in Section 3.3. 


\subsubsection{Airfoil Shape Generator}

In order to generate the airfoil shapes, which can be varied through the use of control points, a Bézier curve was implemented. The position of each of the control points was determined by the gamultiobj optimization routine where each control point can be considered to be a gene (variable). A Bézier curve provides sufficient freedom for the airfoil shape, while ensuring that the curve is smooth in shape such that sharp variations in the airfoil contour are avoided. For these reasons, Bézier curves are commonly used to generate airfoil shapes in optimization routines [78-81,95].

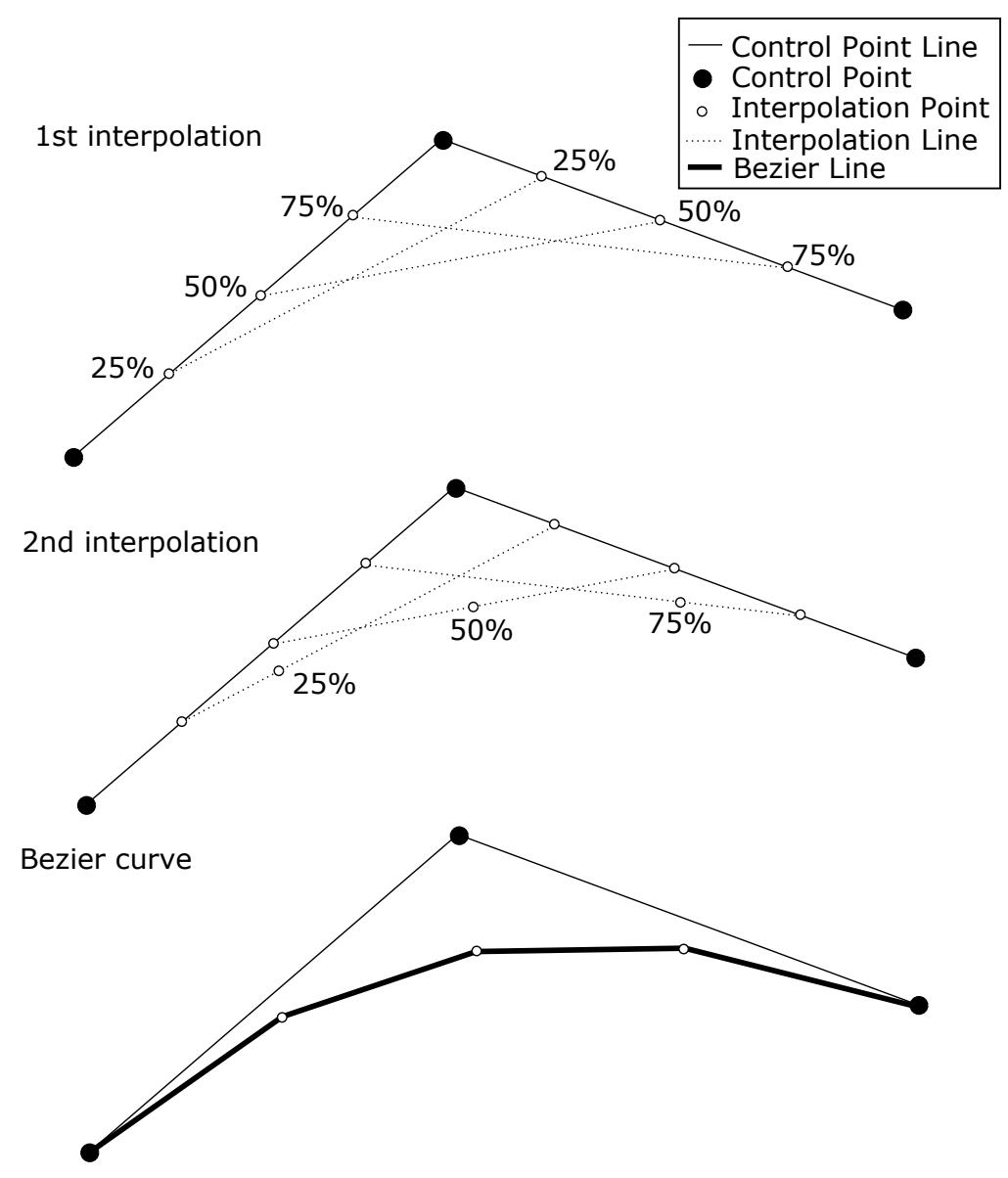

Figure 3.6: Bézier curve generation using 3 control points, and $25 \%$ interpolation steps

A Bézier curve is simply a linear interpolation between control points. As shown 
in Figure 3.6, three control points are used to define an example Bézier curve. A linear interpolation between the two lines connecting the three points is shown here for every $25 \%$ segment of the line. A second interpolation, at $25 \%$ intervals along the interpolated line is performed to obtain points. Interpolations must be performed until individual points are obtained; for curves consisting of more control points, more interpolations will be necessary. These points make up the shape of the Bézier curve. Note that in this example, the resulting curve is not very smooth due to the large interpolation intervals. By using a much smaller interpolation interval, a much smoother curve, consisting of many more points, will result. This geometric interpretation method of evaluating the Bézier curve was proposed by de Casteljau [96] and can be useful when computing power is limited; interpolating between points can potentially be computationally less expensive than evaluating higher order equations [97]. Equation 3.2 shows an analytical formula which can be used to calculate the Bézier curve [97], which in certain applications can be computationally more expensive.

$$
P(z)=\sum_{i=0}^{n} \frac{n !}{i !(n-i) !} z^{i}(1-z)^{n-i} P_{i}
$$

where $n$ is the number of control points minus $1, P_{i}$ is the current control point which acts as a weighting factor, and $z$ is the interpolator which takes on values of $0 \leq z \leq 1$. It can be noted that the general Bézier curve formula employs a summation of Bernstein polynomials, defined as shown in Equation 3.3, along with weighting factors to obtain its shape [96]. In this work, the analytical formula was employed as it was found to be computationally insignificant in relation to the rest of the optimization routine. 


$$
B_{n, i}(z)=\frac{n !}{i !(n-i) !} z^{i}(1-z)^{n-i}
$$

A feature of Bézier curves is that for the start and end control points, that is for $P_{0}$ and $P_{n}$, the first derivative of the curve will be equal to that of the straight line joining the current and adjacent control point. If it is necessary to join two separate Bézier curves with a smooth joint, it is required that the common point between the two curves is sided by collinear points on either side. This ensures that the first derivative between the start and end points of the respective curves are equal at the joining point, thereby leaving a smooth joint.

To describe the shape of an airfoil, one Bézier curve for the top surface, and one Bézier curve for the bottom surface was employed. The use of two separate curves allows for the location of the start and end points- the leading and trailing-edge- to be controlled. Had a single curve been used, the leading-edge could change location effectively altering the chord length of the airfoil and thus requiring scaling such that all generated airfoils have equal chord.

As seen in Figure 3.7, 13 control points are used to describe the shape of the airfoil, which is in agreement with other studies [31,81]. Note that there are actually 14 points, however, the extra point corresponds to the leading-edge of the airfoil which is a common point between top and bottom surfaces, and is therefore a duplicate. Each of the control points are bounded in the vertical and horizontal directions, with the exception of the trailing-edge points which can only move vertically in a symmetric manner about the chord line such that the need to de-rotate the airfoil is eliminated. Additionally, the points adjacent to the leading-edge point are also permitted to move only in the vertical direction; this ensures a smooth joint between the upper and lower Bézier curves at the leading-edge. In total, this results in 18 


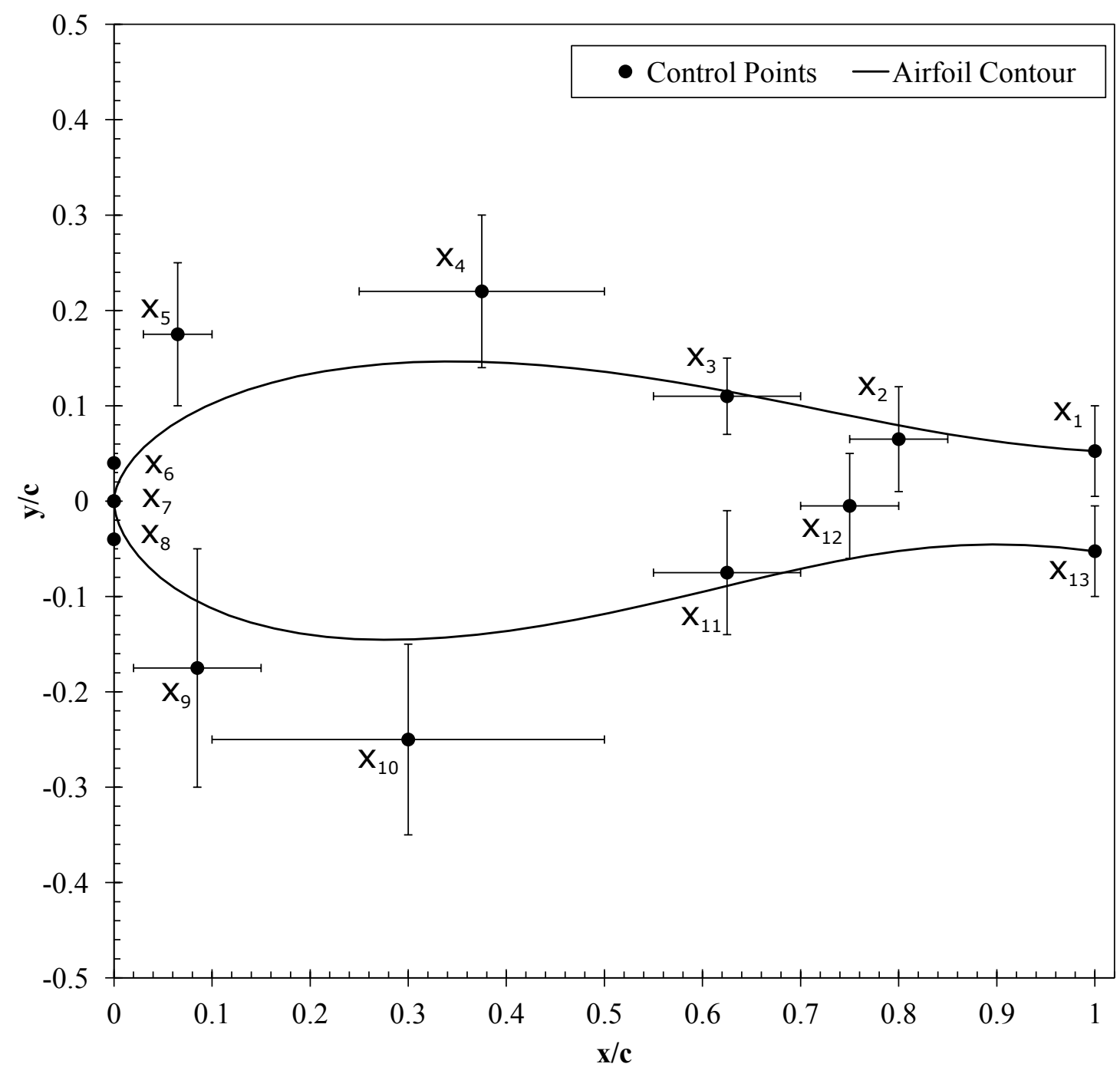

Figure 3.7: Bézier control points used to control the shape of the airfoil

variables (the control points and their respective directions of movement) which is in accordance with other studies $[31,80]$. The bounds of the control points were initially set such that the majority of the design space was covered, however were reduced in size based on several optimization runs. Although large bounds allowed a high level of freedom for airfoil shape, it was found that converged airfoils always took on values that fell within a smaller range. Therefore, reducing the bounds decreases the design space allowing for a more focused optimization and in turn, faster convergence. 
The bounds were adjusted accordingly to allow for airfoils with thicknesses ranging from approximately 20-40\%, and trailing-edge thicknesses ranging from 1-20\%, to be generated.

Constraint functions and inequalities were employed on particular control points to ensure that only realistic airfoil geometries were generated; abnormal airfoil shapes resulted in unconverged solutions in XFOIL and thus should be avoided. One constraint dictated that the value of the upper surface trailing-edge control point was not permitted to take on values greater than that of the point on the suction surface ahead of the trailing-edge. That is:

$$
x_{1}^{V} \leq x_{2}^{V}
$$

where the subscript is the control point number, and the superscript represents either the horizontal or vertical direction of permissible movement. This ensures that the suction surface does not exhibit a "fish tail" towards the trailing-edge which causes convergence issues in XFOIL. Furthermore, the following conditions were imposed which aided in reducing the number of variables, and ensured that the aforementioned symmetric trailing-edge and location of leading-edge point was achieved.

$$
\begin{aligned}
& x_{6}^{V}=-x_{8}^{V} \\
& x_{1}^{V}=-x_{13}^{V} \\
& x_{7}=(0,0)
\end{aligned}
$$




\subsubsection{Coupler}

With the shape of the airfoil defined, its aerodynamic and structural characteristics must be evaluated such that the optimization scheme can determine its performance relative to the other designs. In order to do so, there must exist a means of running an external program, in this case XFOIL, extracting the necessary information, and providing it back to MATLAB's gamultiobj for evaluation. An existing open-source MATLAB script [98] was used and modified as necessary for this work. The script provides XFOIL with the necessary information for the simulations as inputs, and saves the output aerodynamic data to a temporary file. The information from this file, notably the $C_{L}, C_{D}$, and $C_{M}$ associated to each $\alpha$, is read by MATLAB and used to evaluate the airfoil's aerodynamic fitness in the optimization scheme. This data is read using a custom script, which had to be robust in order to deal with non-typical convergence issues with XFOIL. For every new airfoil design, XFOIL is run twice; once for free transition conditions, and once for forced transition conditions.

In addition to the calculation of the aerodynamic performance of the airfoil using XFOIL coupled with MATLAB's gamultiobj, the structural performance of the airfoil can be determined numerically based on the airfoil's shape. As described earlier in the thesis, the sectional moment of inertia of an object, $I_{X X}$, is a measure of its resistance to bending about an axis. Due to the slenderness of airfoils, the majority of bending will arise in the flapwise direction i.e. about the chordline, in the $y$-direction. For a 2D object, Equations 3.8-3.10 can be used to evaluate the $I_{X X}$ [63]. Note that these equations assume a uniform material distribution and properties across the entire 2D shape. Only the flapwise sectional moment of inertia $\left(I_{X X}\right)$ was considered in this work, however, as rotor blades increase in length and weight, the gravitational loads become more of a concern [1] and the inclusion of the edgewise sectional moment of inertia $\left(I_{Y Y}\right)$ and torsional stiffness $(J)$ may be included as well. 


$$
\begin{gathered}
A=\int_{0}^{c}\left(y_{u}-y_{l}\right) \partial x \\
\bar{y}=\frac{1}{A} \int_{0}^{c} \frac{1}{2}\left(y_{u}^{2}-y_{l}^{2}\right) \partial x \\
I_{X X}=\int_{0}^{c} \frac{1}{3}\left[\left(y_{u}^{3}-\bar{y}\right)-\left(y_{l}^{3}-\bar{y}\right)\right] \partial x
\end{gathered}
$$

As can be seen, the moment of inertia calculation takes into account the distribution of material (the area, $A$ ) about the neutral line of the airfoil $(\bar{y})$. An increased airfoil thickness, or trailing-edge thickness, will inevitably increase the distance of the upper and lower airfoil surfaces ( $y_{u}$ and $y_{l}$, respectively) from the neutral line, thereby resulting in a stiffer, and structurally better performing airfoil in the flapwise direction. The $I_{X X}$ of each airfoil is evaluated and used as the structural measure of its performance in the design optimization process.

\subsubsection{XFOIL}

XFOIL is a two-dimensional airfoil analysis and design tool developed by Drela [25] at the Massachusetts Institute of Technology in 1986. For a user-input set of coordinates which define the airfoil shape, XFOIL is capable of predicting the aerodynamic performance at a variety of Reynolds, Mach, turbulence, and transitional conditions. XFOIL employs a viscid-inviscid formulation in which the inviscid flow is calculated using a panel method and combined with the viscid component, which is calculated using boundary layer integral equations. Despite XFOIL's over-prediction of lift and under-prediction of drag in the stall and post stall regions [60-62,99], its very fast computational time and accurate predictions in the pre-stall region make it an ideal 
tool in the optimization of airfoils. It should be noted that XFOIL only formulates steady-state solutions. If unsteady aerodynamic effects are desired to be studied, methods such as the Beddoes-Leishman model [100] may be implemented.

\section{Inviscid Formulation}

Panel methods make use of several small panels which define the outer surface of any shape, in this case a 2D airfoil section. Each panel has an associated vortex strength, typically calculated at the mid-point of the panel. This vortex strength can be constant across the panel, or vary spatially. XFOIL utilizes a linear vortex panel method in which the strength varies linearly between adjacent panels. Each of the panels will experience induced velocities due to the presence of the surrounding vortex panels, and in order to ensure that the boundary condition of zero velocity normal to the panel (ensuring solid outer surface condition) is satisfied, the strength of the vortex panels must be calculated such that the induced velocities, plus the component of velocity due to the freestream result in zero velocity normal to the panel.

For thin trailing-edge airfoils, the Kutta condition, which states that the flow must leave the trailing-edge smoothly [21], must also be satisfied. In order to satisfy this condition the velocity, and therefore vortex strength, must be equal between the top and bottom panels which share a coincidental point at the trailing-edge. This introduces an additional equation that when combined with the zero normal velocity equations, creates an overdetermined system. Therefore, in order to solve for the vortex strengths of the panels, one of the control points along the airfoil must be ignored. In the case of airfoils with thick trailing-edges, this step need not be taken as there is no coincidental point at the trailing-edge. An additional panel is placed at the thick trailing-edge with a vortex strength equal to the average between the adjacent top and bottom surface panels [25]. 
With the vortex strength at each panel calculated, the tangential velocity can be determined and from that, assuming incompressible flow, the pressure distribution over the airfoil can be computed. For thin airfoils at low angles of attack, this inviscid formulation provides results that are near experimental values, as the flow is still attached and the thin boundary layer has little effect. However, at higher angles of attack and where separation becomes more prominent, viscous effects must be accounted for. [21]

\section{Viscid Formulation}

With the inclusion of friction effects, boundary layers will begin to develop at the leading-edge of the airfoil. These boundary layers will displace the inviscid flow around the airfoil, thereby altering its effective shape. Knowing the outer-edge velocity of the boundary layer from the inviscid solution, the equations used to determine the viscous boundary layer properties can be solved [101]. Integral boundary layer equations are used to determine both the boundary layer displacement thickness, as well as the momentum thickness which represent the distance for which the outer, inviscid flow, is displaced in the direction normal to the body surface, and the hypothetical height of the streamline required to makeup for the lost momentum in the boundary layer, respectively. The inviscid solution is thus displaced outwards using source panels along the airfoil surface.

Once the boundary layer characteristics have been calculated, the $e^{N}$ transitional model can be used to predict the transition to turbulence point on the upper and lower surfaces of the airfoil. The $e^{N}$ transitional model uses semi-empirical data to predict the point at which the instability point- the point at which Tollmien-Schlichting waves develop- occurs and is based on linear-stability theory [102]. Depending on the level of freestream turbulence, the amplification factor, $N$, which is a measure 
of the current wave amplitudes in relation to the critical wave amplitudes at which transition occurs, can be modified to adjust the prediction of transition. For wind tunnels with freestream turbulence levels $\leq 0.1 \%$ it has been found that $N=9$ is typically an appropriate value. According to Mack [103], the following relation shown in Equation 3.11 can be used to calculate the approximate value of $N$ based on the freestream turbulence levels. Although this formula gives a general indication of the $N$ factor to be used, the prediction of transition also depends on things like the frequency of the instability waves, as well as any acoustic disturbances. [102]

$$
N=-8.43-2.4 \ln T u
$$

The sensitivity of the aerodynamic performance prediction of $C_{L \text { Max }}$ and $C_{D \text { Min }}$, with respect to the number of panels used in XFOIL is shown in Figure 3.8, for a 30\% thick, flatback airfoil (CU-W1-30, see Section 3.6). Based on this study, 160 panels were used in all XFOIL analyses; more panels result in differences in lift and drag of about $0.5 \%$ which can be considered insignificant.

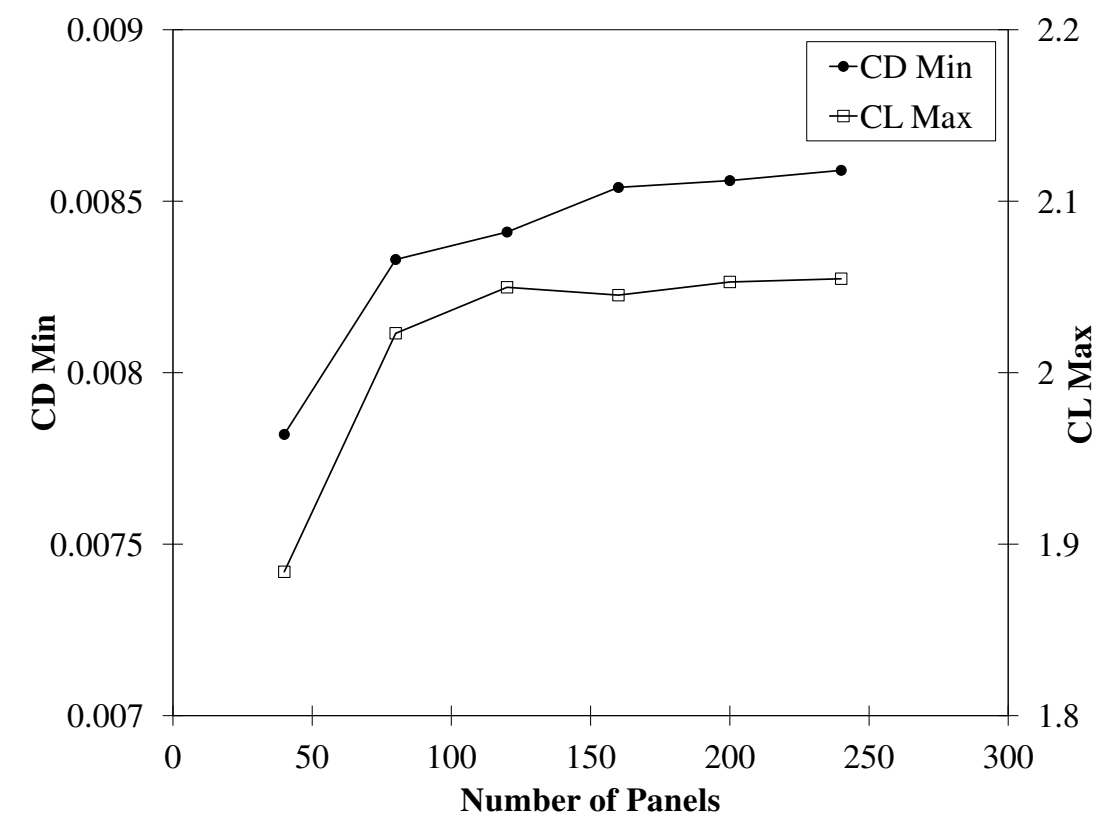

Figure 3.8: Sensitivity of $C_{L M a x}$ and $C_{L \text { Min }}$ with respect to the number of panels used in XFOIL 
The aforementioned shortcomings of XFOIL in the stall and post-stall region of the aerodynamic performance predictions can be shown in Figures 3.9 and 3.10. However, before stall $\left(A O A<10^{\circ}\right)$, the predictions are quite accurate. These results have been obtained for a S809 wind turbine airfoil at Carleton University by the current author, and are further described in Chapter 4.

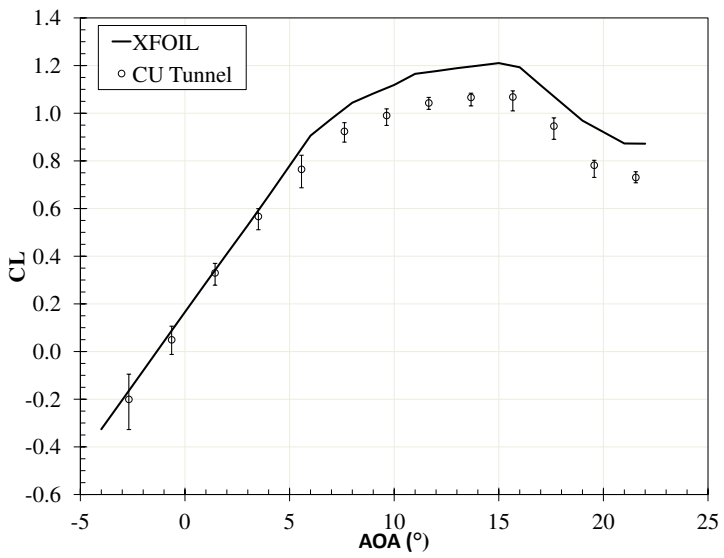

Figure 3.9: Comparison of XFOIL lift predictions and experimentally measured values for S809 airfoil

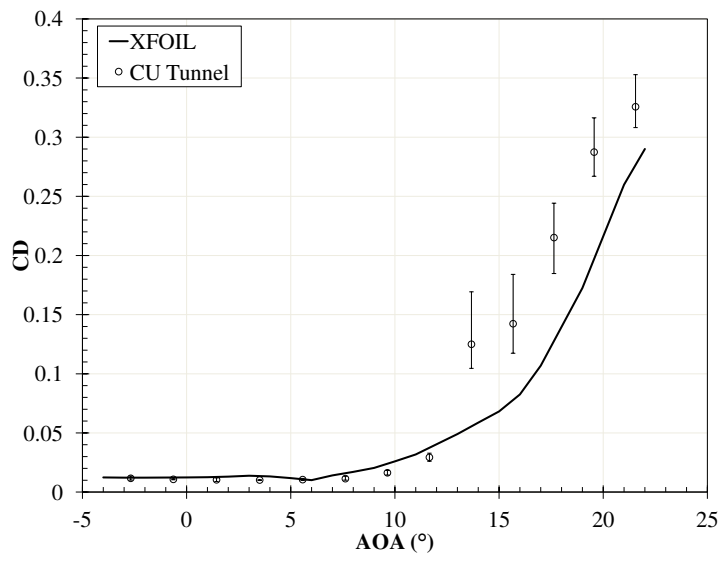

Figure 3.10: Comparison of XFOIL drag predictions and experimentally measured values for S809 airfoil

In summary, XFOIL provides robust, fast, and relatively accurate predictions of the aerodynamic forces acting on 2D airfoils under a variety of operating conditions. For largely separated flow, XFOIL predictions tend to diverge from experimental values, however improvements to the prediction of stall and post-stall values have been implemented in a modified XFOIL code named RFOIL. Although RFOIL is likely better suited for the prediction of thick airfoils with thick trailing-edges, the necessary permissions and access to the code were not able to be attained for this work. 


\subsection{Convergence Analysis}

In order to ensure that the gamultiobj optimizer solutions were converged, convergence analyses were performed for the number of generations, as well as the population size. One of the aforementioned variables was varied, while the other was kept constant. The value that was monitored was taken as the optimal combination of $C_{L} / C_{D}$ and $I_{X X}$ performance which was evaluated by normalizing the $C_{L} / C_{D}$ and $I_{X X}$ values with respect to their maximums, and finding the distance of each of the individuals from the origin (root mean square), as shown in Equation 3.12 and as illustrated in Figure 3.11. The individual which is farthest in distance from the origin has the largest root mean square (RMS) value, and in theory should possess the best possible trade-off between aerodynamic and structural performance.

$$
R M S=\sqrt{\left(C_{L} / C_{D \text { Norm }}\right)^{2}+\left(I_{X X N o r m}\right)^{2}}
$$

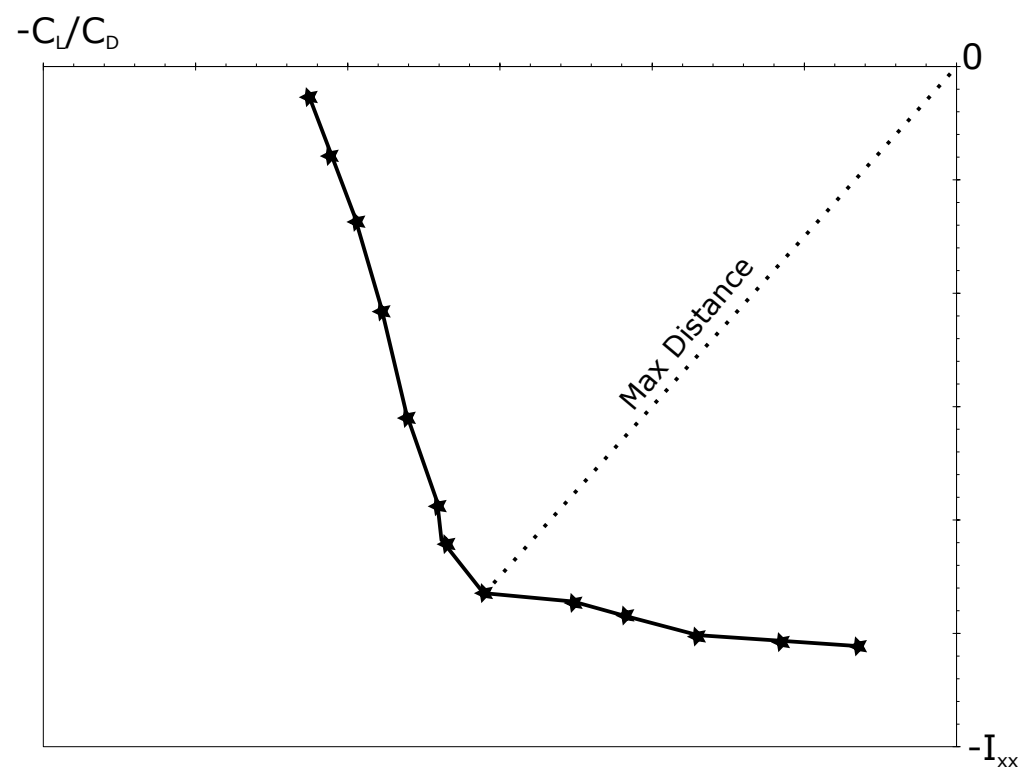

Figure 3.11: Sample Pareto front showing an individual with the largest RMS value

With a constant value of 100 generations, the population size was varied from 
50-500. As seen in Figure 3.12, as the population size was increased, an asymptotic behavior in the RMS value was observed suggesting convergence. Although population sizes greater than 200 tended to show a slight increase, all values of RMS were within $1 \%$ of the value found at a population level of 200 . Furthermore, a population size of e.g. 500 would take approximately $2.5 \times$ the computational time, and only come with less than a $1 \%$ increase in RMS. Therefore a population size of 200 was chosen which is in-line with other optimization studies with a similar number of problem variables $[104,105]$ as well as the general formulation derived by De Oliveira [106] shown in Equation 3.13 where numvar is the number of variables (18 in this work) and $O b j$ is the number of objectives ( 2 in this work).

$$
\text { Population }=(10 \times \text { numvar })^{\text {Obj-1 }}
$$

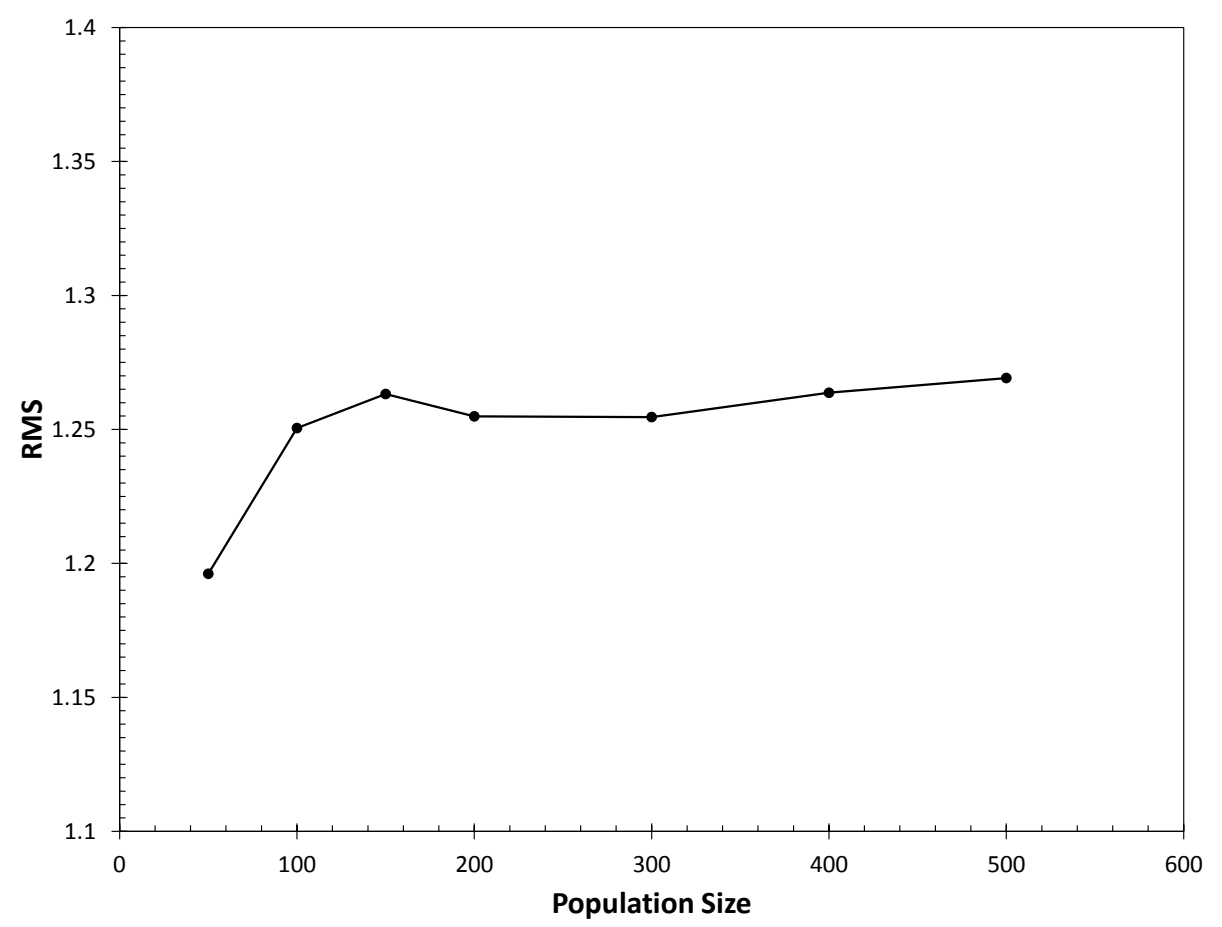

Figure 3.12: Results of the convergence analysis when varying population size 
With the population size set at 200, the number of generations was then varied. As shown in Figure 3.13, changing the number of generations resulted in little-tono change in the RMS value. For generation sizes larger than 150, the gamultiobj solver oftentimes stopped itself before the maximum set number of generations was reached due to very little change in the values of the pareto front, indicating converged solutions. A generation size of 100 was chosen for all studies.

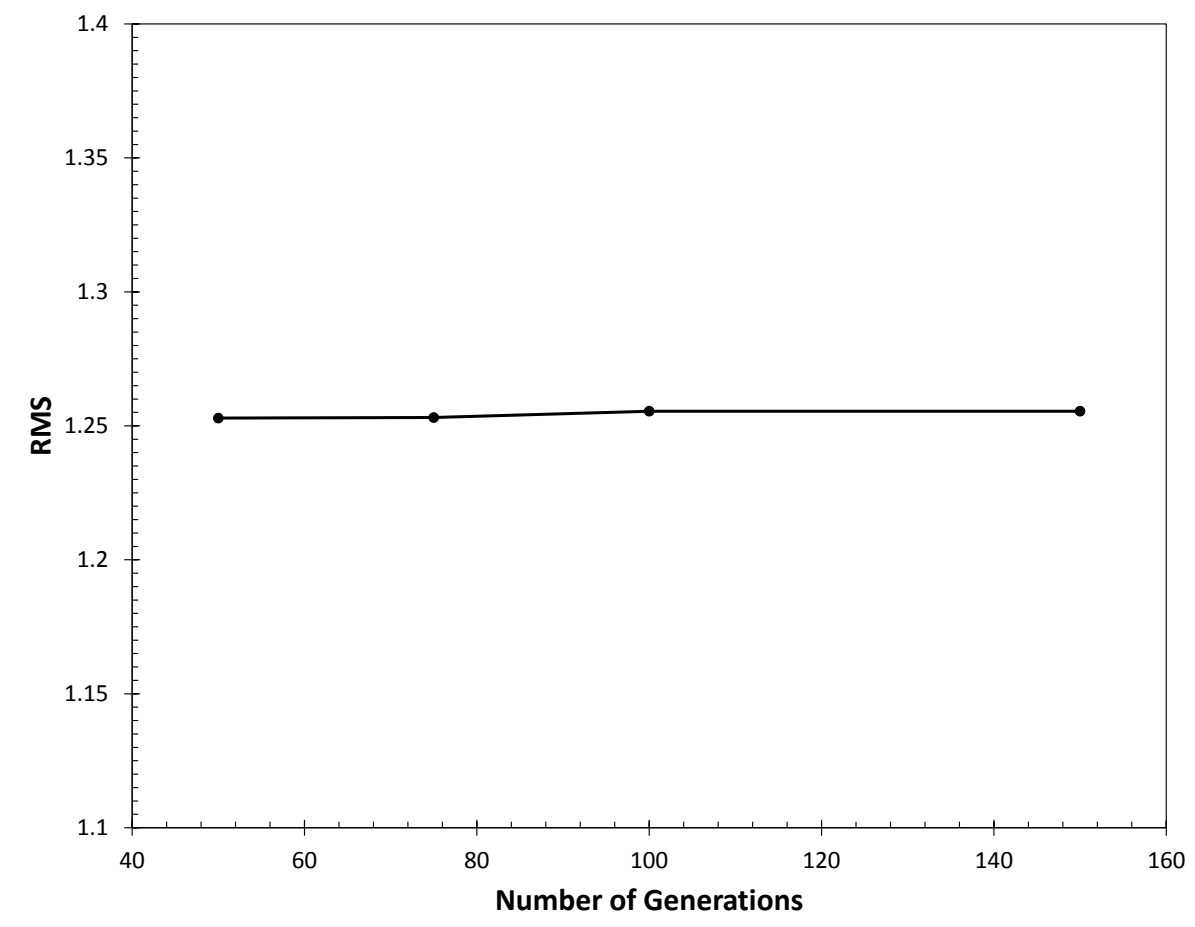

Figure 3.13: Results of the convergence analysis when varying number of generations

These convergence analyses have shown that the population size has a much greater influence on the optimization outcome than the number of generations. If a very small population had been used, it is likely that only a small portion of the design space would have been explored, and in turn, converged results would be established relatively quickly. However, large population sizes ensure that the entire 
design domain is explored, requiring more computations and accordingly longer convergence times. Therefore, it is essential that adequate and reasonable population sizes and numbers of generations be used for all optimization runs; in this case 200 and 100, respectively.

\subsection{Airfoil Optimization}

The optimization of wind turbine airfoils was performed using the aforementioned developed tool. The work performed in the following sections aims to analyze how the variation of various design parameters impacts the airfoil design outcome. With the obtained knowledge, informed design decisions can be made such that airfoils are properly optimized. The following sections will highlight the effect of varying aerodynamic evaluators, a sensitivity analysis performed using the Taguchi method, and the design of an airfoil family.

\subsubsection{Design and Optimization Parameters}

In order to simulate the operational conditions of multi-MW wind turbine airfoils found in the inboard region of the blade [4,107], $R e=6 \times 10^{6}$ and $M a=0.1$ was used in XFOIL. Furthermore, an N-crit number of 9, representing typical wind tunnel turbulence values, was used for all simulations. For a given airfoil, XFOIL was ran once under clean conditions (free transition) and then again under tripped conditions (forced transition). The forced transition location was specified in XFOIL to occur at $2 \%$ from the leading-edge on the suction surface, and $10 \%$ on the pressure surface, which are realistic values for contaminated leading-edges found on in-service wind turbines [108-111]. This forward-located forced transition mimics a high degree of leading-edge roughness from contamination, erosion or manufacturing defects which 
trip the flow from laminar to turbulent conditions.

Insensitivity to leading-edge roughness was ensured by comparing the airfoil's lift performance under free and forced transition conditions; the largest differences were found near $C_{L}$ Max. If the airfoil's $C_{L}$ decreases by less than $15 \%$ under forced transition conditions, it was accepted as a suitable airfoil to be compared to the others. However, if the difference was greater than $15 \%$, a penalty was imposed on the aerodynamic performance of the airfoil by dividing its aerodynamic fitness function value by 100 . Originally a value of 0 was assigned to the airfoils not meeting this criteria as a penalty, however, it was found that the optimizer had trouble determining in which direction the suitable solutions lie. A non-zero value ensured that the optimizer had sufficient feedback from the various airfoil iterations to determine that a given airfoil is good, however, simply did not meet one of the criteria.

This roughness insensitivity constraint on free versus forced transition performance results in the free transition point naturally moving to a location near the leading-edge at angles of attack close to $C_{L \text { Max }}$. A forward located free transition point near $C_{L \text { Max }}$ means that very little difference in performance will arise when transition is forced as a result of leading-edge roughness. At the inboard region of the blade, airfoils typically operate at about $7^{\circ}$ angle of attack [112], however, is dependent on the conditions in which the turbine operates. Therefore, an angle of attack range of $7 \pm 3^{\circ}$ was taken as the operational range and accounts for fluctuating angle of attack due to wind gusts and off-design conditions.

A constant thickness of $30 \%$ was used for all airfoils in the study performed to determine the effect of the aerodynamic evaluators. This thickness was chosen because it is thick, yet still rather accurately solvable by XFOIL, it is commonly found in literature, and is a thickness likely to be more prominent as rotor blades increase in length. This constant thickness ensures that all airfoils can be compared equally 
without the relative thickness playing a role in the results. In the Taguchi sensitivity study, and in the design of the airfoil family, the thickness and operational $\alpha$ range were allowed to take on different values as specified. Since these studies are solely concerned with the optimization of the airfoil itself, and not the rotor blade as a whole, the effects of the airfoil camber on the optimization were eliminated by taking all performance values relative to the zero-lift angle of attack $\left(\alpha_{C_{L 0}}\right)$ as shown in Equation 3.14 [78].

$$
\alpha=\alpha_{\text {original }}-\alpha_{C_{L}}
$$

The reason why the effects of airfoil camber should be ignored is that they can be compensated for in the design of the rotor blade by varying the geometric twist of the blade such that the airfoil operates at its ideal conditions [78]. In the design of a rotor blade, designers oftentimes try to achieve a certain level of $C_{L}$ along the span of the blade such that the blade is appropriately aerodynamically loaded. A cambered airfoil essentially shifts the linear portion of the lift curve when compared to a symmetric airfoil which means that at a given angle of attack, the cambered airfoil will exhibit higher levels of lift than the symmetric airfoil. However, as mentioned earlier, the blade twist will be adjusted in the blade design such that the blade produces appropriate levels of lift and drag, and in turn is properly aerodynamically loaded.

Recall that in the calculation of coefficient of torque and thrust $\left(C_{T}=C_{L} \sin \phi-\right.$ $C_{D} \cos \phi$ and $C_{N}=C_{L} \cos \phi+C_{D} \sin \phi$, respectively) the flow angle is a function of both the angle of attack and blade twist $(\phi=\alpha+\beta)$. For a given level of lift, the cambered airfoil will operate at a lower angle of attack than the symmetric airfoil, and the difference will be compensated for by adjusting the blade twist. However, in 
the optimization of the airfoils for this study, the blade twist was assumed to be zero $(\beta=0)$ therefore $\phi=\alpha$. By making use of the $\alpha$ as defined in Equation 3.14, it is ensured that $C_{T}$ and $C_{N}$ are properly calculated to account for the effects of camber. With all other factors set for equal comparison, the differences in airfoil performance for the various aerodynamic evaluators should come as a result of only trailing-edge thickness and airfoil shape.

An integral of the weighted-sum performance for a given aerodynamic evaluator under free and forced transition conditions over the optimized $\alpha$ range was used for comparison between the airfoils. A $25 \%$ free, and $75 \%$ forced transition weightedsum was chosen such that a situation where the majority of the turbine's life is spent under rough conditions is properly represented [113]. This is of particular importance as blade lengths continue to increase, and thicker airfoils become more prominent at larger radii where fouling is typically found. Note that all optimization runs were repeated 3 times to verify and ensure that results were consistent.

\subsubsection{Aerodynamic Evaluator Results}

The term aerodynamic evaluator can be used to describe the specific aerodynamic objective used during the optimization scheme. Typically, $C_{L} / C_{D}$ is chosen as the aerodynamic evaluator which is to be maximized, however, in the optimization of wind turbine specific airfoils, it is possible to implement aerodynamic evaluators such as $C_{T}$, and $C_{T} / C_{N}$. The influence of the aerodynamic evaluator on the airfoil shape and performance will be presented below.

\section{Comparison of Airfoil Shape and Performance}

Three airfoils with a maximum thickness of $30 \%$, individually optimized for $C_{L} / C_{D}$, $C_{T}$, and $C_{T} / C_{N}$ were generated using the flatback airfoil aero-structural optimizer 
developed in this work. As can be seen in Figure 3.14, in comparison to the very similar $C_{L} / C_{D}$ optimized and $C_{T} / C_{N}$ optimized airfoils, the $C_{T}$ optimized airfoil has a different pressure surface shape and the shallowest suction surface. The $C_{T}$ optimized airfoil's maximum thickness is located at the $28 \%$ chord location, and features a $4.8 \%$ thick flatback trailing-edge whereas the $C_{L} / C_{D}$ and $C_{T} / C_{N}$ optimized airfoils both possess a maximum thickness at the $33 \%$ chord location and a $3.9 \%$ trailingedge thickness. Despite these differences, all three airfoils can be considered to be structurally equivalent since they possessed comparable values of $I_{X X}$.

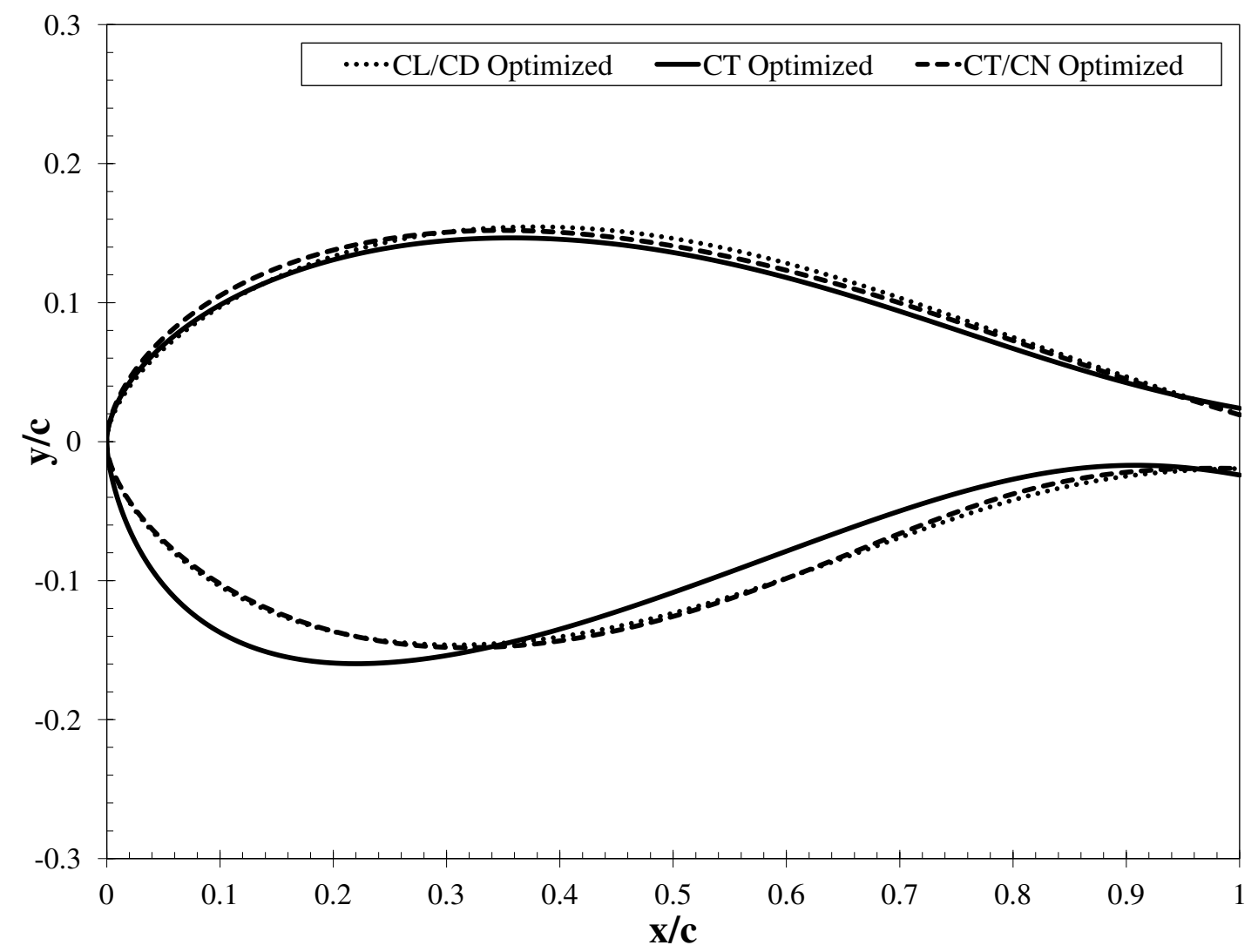

Figure 3.14: Three $30 \%$ thick airfoils optimized for maximum $C_{L} / C_{D}, C_{T}$, and $C_{T} / C_{N}$ 


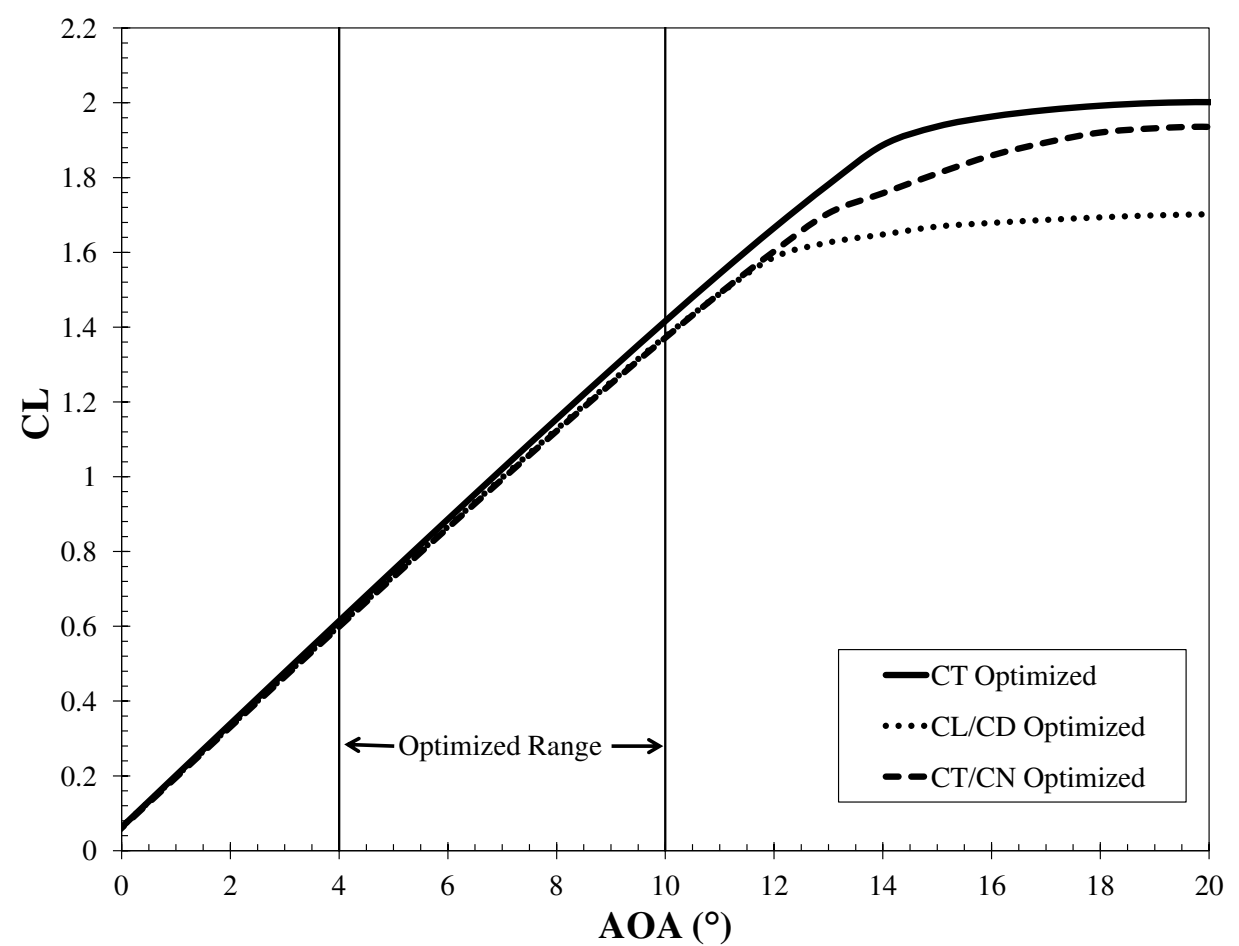

Figure 3.15: $C_{L}$ of the airfoils under free transition conditions at $R e=6 \times 10^{6}$

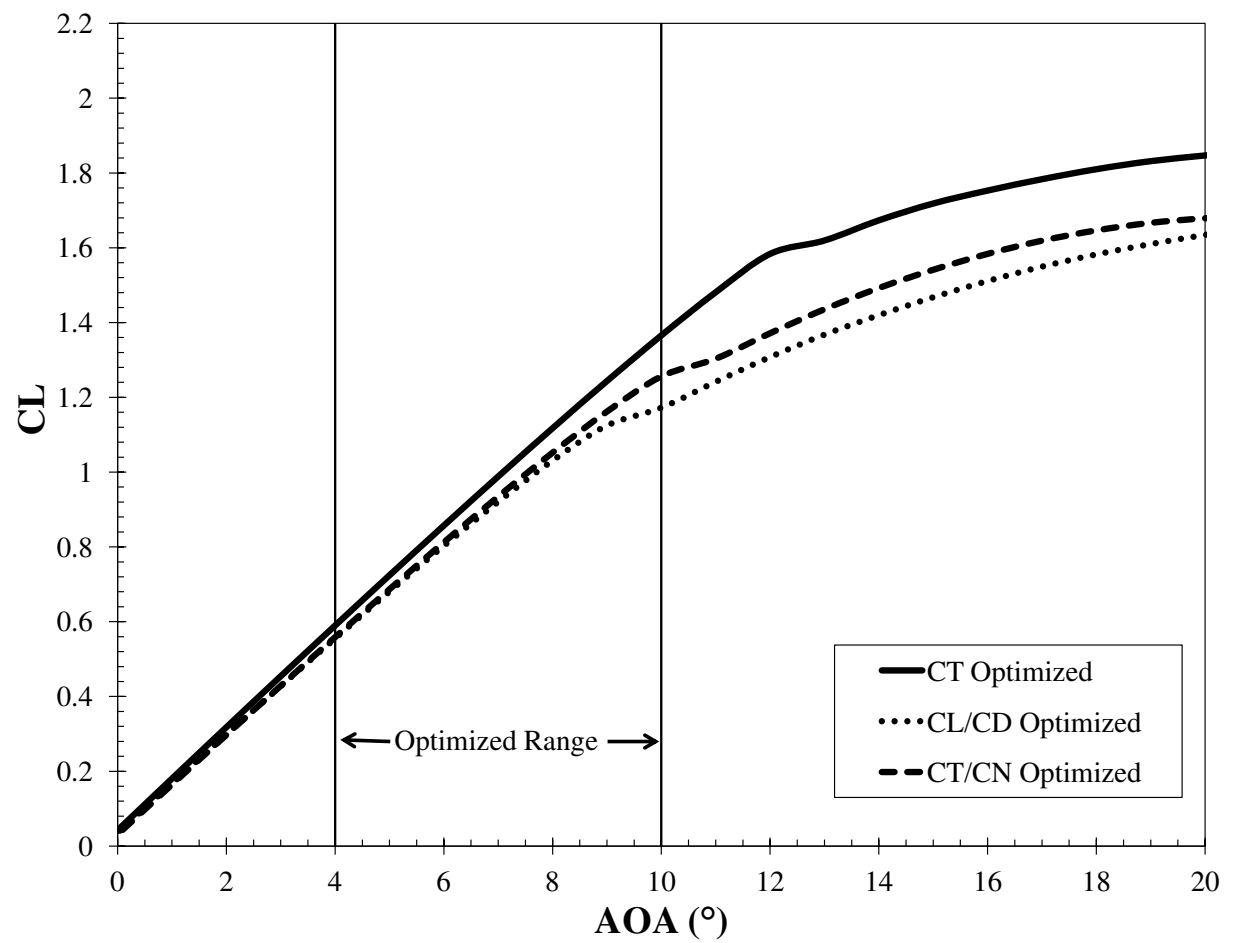

Figure 3.16: $C_{L}$ of the airfoils under forced transition conditions at $R e=6 \times 10^{6}$ 


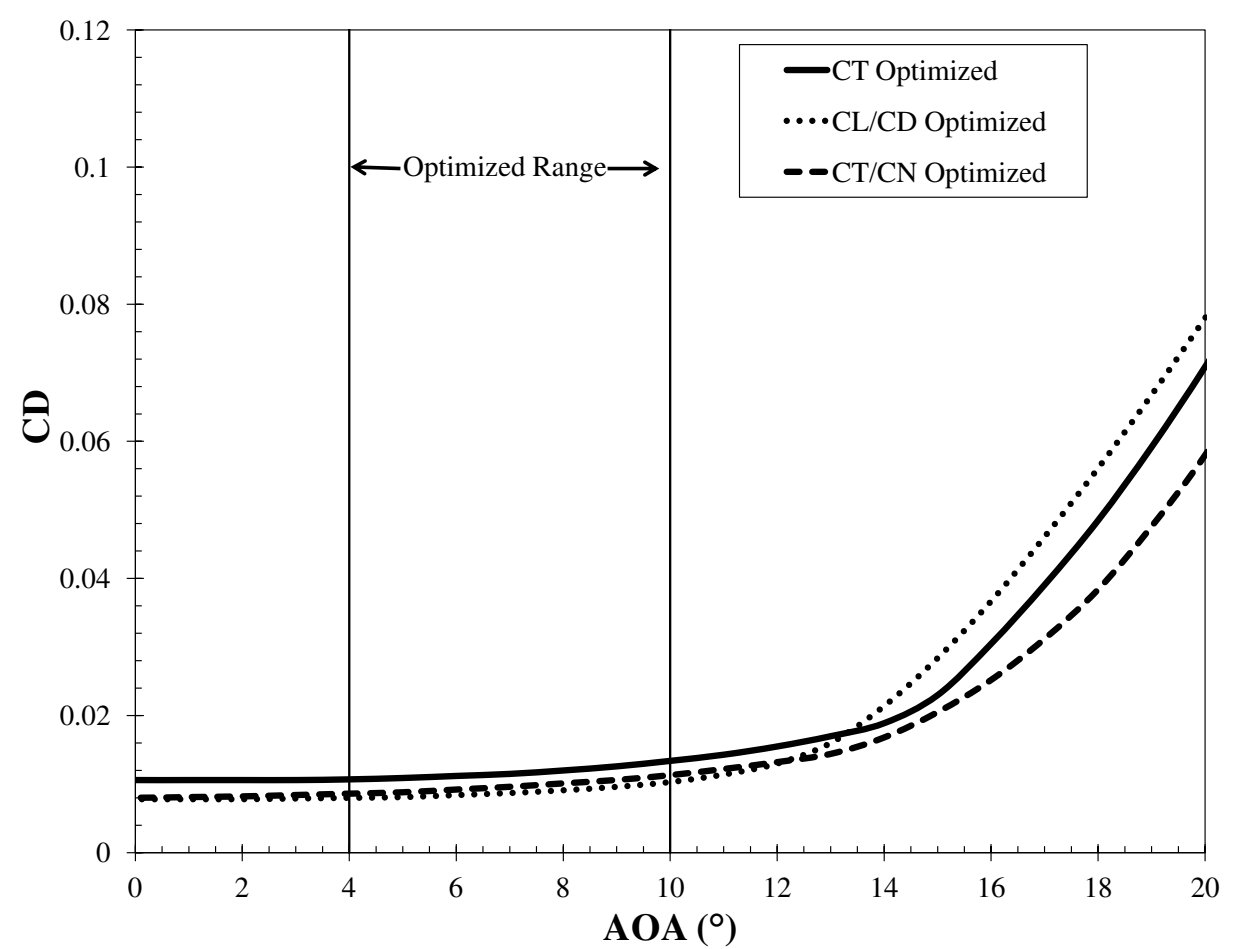

Figure 3.17: $C_{D}$ of the airfoils under free transition conditions at $R e=6 \times 10^{6}$

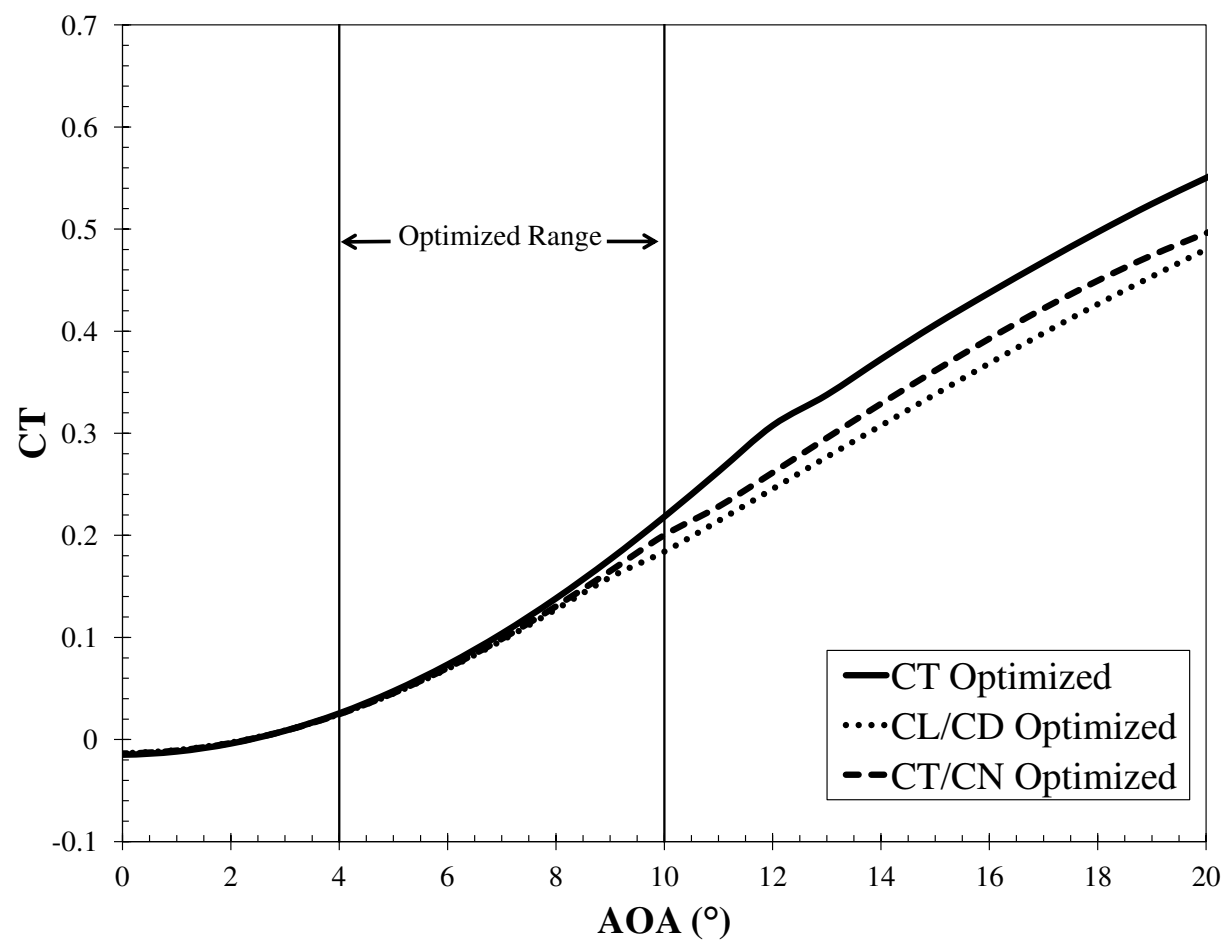

Figure 3.18: $C_{D}$ of the airfoils under forced transition conditions at $R e=6 \times 10^{6}$ 
Figures 3.15 and 3.16 show the $C_{L}$ performance of all three airfoils under free and forced transition as predicted by XFOIL. In both cases, the $C_{T}$ optimized airfoil exhibits the largest $C_{L}$ values thanks to its slightly larger trailing-edge thickness, which forces the pressure recovery to occur in the wake, as well as its larger camber value of $2.2 \%$ compared to the $1.8 \%$ of the remaining two airfoils. Under forced conditions, the drop in $C_{L}$, and inevitable increase in $C_{D}$ for the $C_{T}$ optimized airfoil is the least significant of all airfoils showing that the large trailing-edge, as well as the shallow suction surface, results in good insensitivity to roughness. As shown in Figures 3.17 and 3.18 , large values of $C_{D}$ come as a by-product of not only the thicker trailing-edge, but also due to the more forward-located free transition points on both the suction and pressure surfaces compared to the $C_{L} / C_{D}$ and $C_{T} / C_{N}$ optimized airfoils. The $C_{L} / C_{D}$ airfoil possesses the most aft-located transition points meaning that under free transition conditions, a large portion of the airfoil exhibited laminar flow thereby aiding in the reduction of drag values.

When comparing the $C_{L}$ and $C_{D}$ performance of the $C_{L} / C_{D}$ and $C_{T} / C_{N}$ optimized airfoils, it can be seen that the $C_{T} / C_{N}$ airfoil produces more lift, as well as drag due to the slightly more blunt leading-edge. This blunter leading-edge results in higher coefficients of pressure around the leading-edge region, thereby producing a more adverse pressure gradient, and in turn, a more forward-located free transition point on the suction surface compared to the $C_{L} / C_{D}$ airfoil. However, under tripped conditions, this already forward-located transition point of the $C_{T} / C_{N}$ airfoil leads to a less drastic drop in performance; the lift remains relatively high and the drag does not increase greatly. The $C_{L} / C_{D}$ optimized airfoil has a large portion of laminar flow over the top and bottom surfaces which helps reduce drag, however, leads to poor relative performance when transition is forced. In other words, the $C_{L} / C_{D}$ airfoil showed inferior insensitivity to roughness under the given conditions compared to the 
other two airfoils. All three airfoils exhibit rather similar gentle stall characteristics, with no sudden loss of lift in the examined AOA range.

Table 3.1: Key aerodynamic characteristics for the three airfoils. Numbers in brackets represent the percent change under forced transition

\begin{tabular}{|c||c|c|c|}
\hline \multicolumn{4}{|c|}{ Key Airfoil Characteristics } \\
\hline Optimized For: & $C_{L} / C_{D}$ & $C_{T}$ & $C_{T} / C_{N}$ \\
\hline$C_{L} @ 7^{\circ}$ & $1.00(-8.0 \%)$ & $1.02(-3.2 \%)$ & $0.99(-6.1 \%)$ \\
$C_{D} @ 7^{\circ}$ & $0.0087(+55.0 \%)$ & $0.0115(+38.0 \%)$ & $0.0096(+46.4 \%)$ \\
Free trans. top @ $7^{\circ}(\mathrm{x} / \mathrm{c})$ & 0.407 & 0.338 & 0.317 \\
Free trans. bottom $@ 7^{\circ}(\mathrm{x} / \mathrm{c})$ & 0.480 & 0.330 & 0.488 \\
\hline
\end{tabular}

Table 4.1 highlights some key characteristics of each airfoil, with the number in brackets representing the percent change in the value under forced transition conditions. The airfoil optimized for $C_{T}$ experiences the smallest reduction in lift, and the smallest increase in drag compared to the other two airfoils under tripped conditions. As mentioned earlier, this is due to the already forward-located free transition points which minimizes the diminishing performance effects of the simulated leadingedge roughness. The $C_{T} / C_{N}$ optimized airfoil demonstrates leading-edge roughness insensitivity that is in between that of the $C_{L} / C_{D}$ and $C_{T}$ optimized airfoils.

\section{$\mathrm{C}_{\mathrm{L}} / \mathrm{C}_{\mathrm{D}}$ Performance}

Figures 3.19-3.20 show the free and forced transition performance, while Figure 3.21 shows the weighted-sum performance ( $25 \%$ free, $75 \%$ forced transition) of the three 


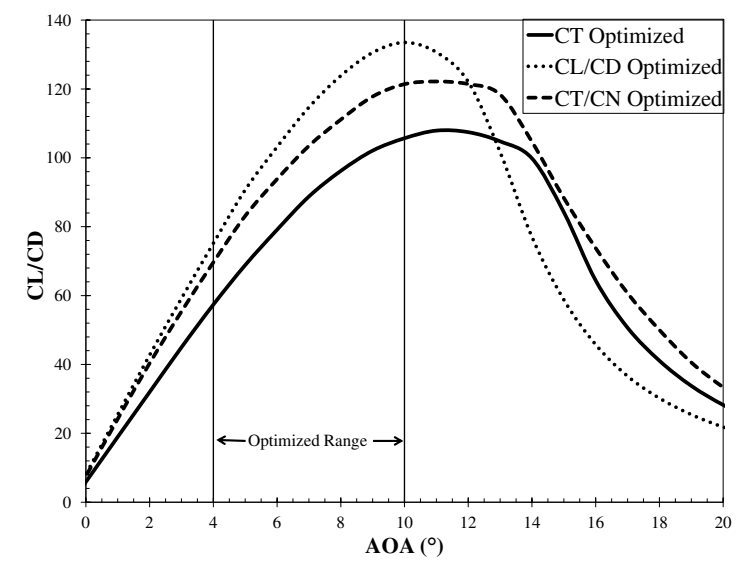

Figure 3.19: $C_{L} / C_{D}$ of the three airfoils under free transition conditions at $R e=6 \times 10^{6}$

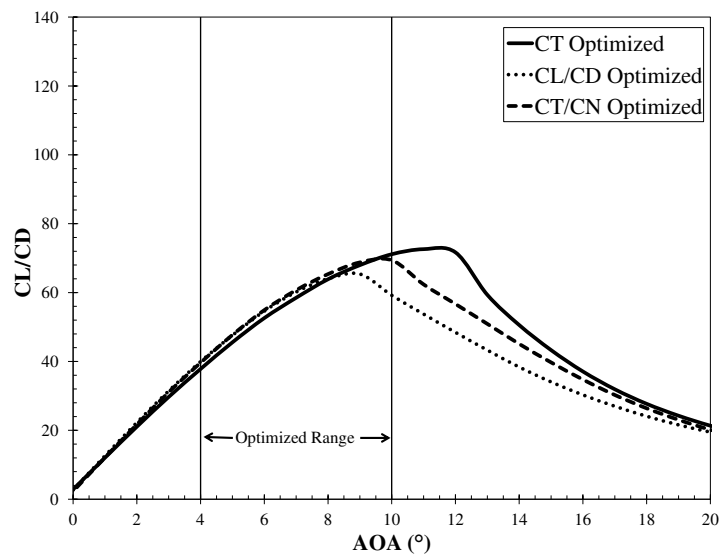

Figure 3.20: $C_{L} / C_{D}$ of the three airfoils under forced transition conditions at $R e=6 \times 10^{6}$

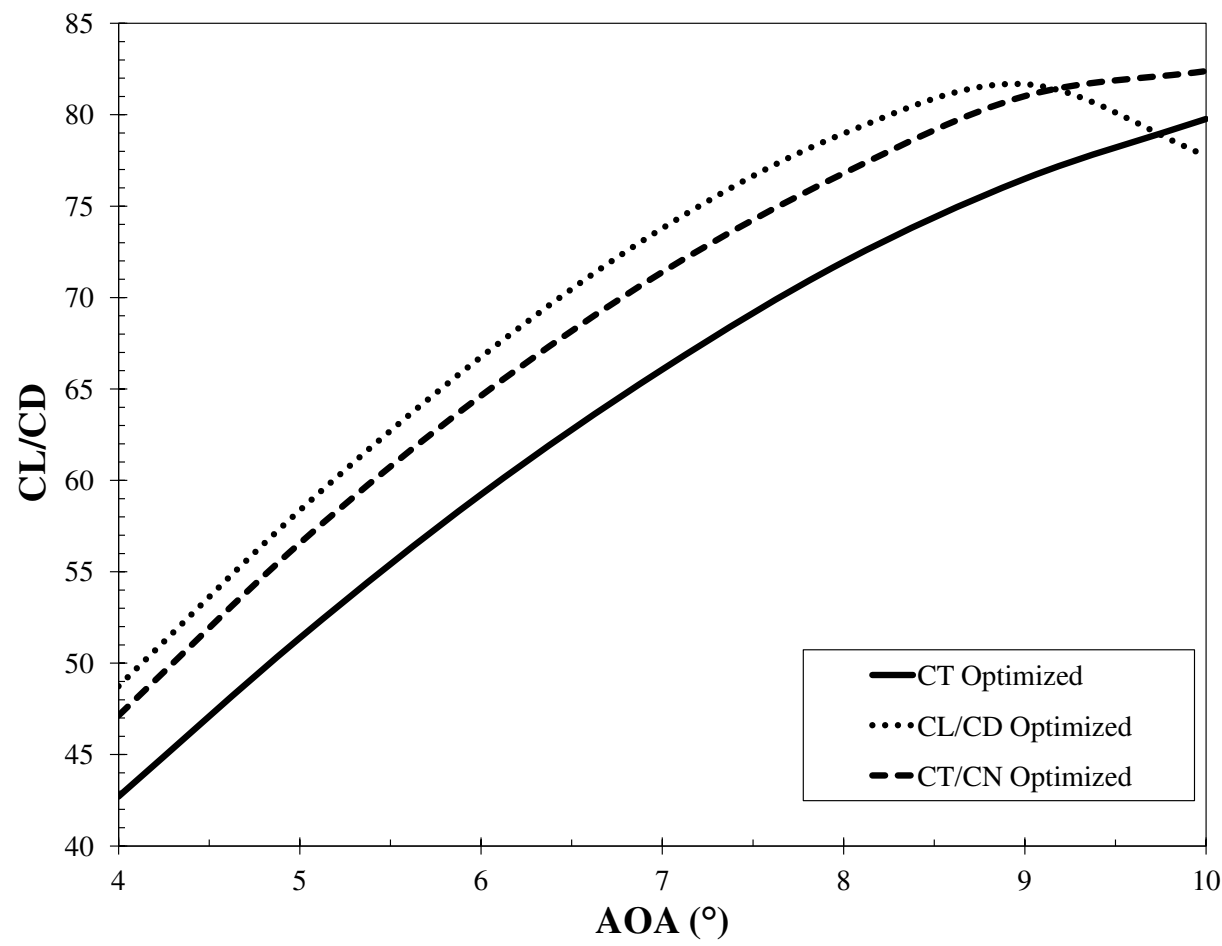

Figure 3.21: The $C_{L} / C_{D}$ weighted-sum ( $25 \%$ free, $75 \%$ forced transition) performance of each of the three airfoils in the $\alpha$ range 
airfoils in the optimized $\alpha$ range when $C_{L} / C_{D}$ is assigned as the aerodynamic evaluator. Despite differences in lift and drag due to varying degrees of roughness insensitivity, the $C_{L} / C_{D}$ and $C_{T} / C_{N}$ optimized airfoils performed similarly when the integral of the $C_{L} / C_{D}$ curve over the weighted-sum $\alpha$ range was taken, with the $C_{L} / C_{D}$ slightly outperforming the rest. The $C_{T}$ optimized airfoil showed the poorest $C_{L} / C_{D}$ performance due to its large values of $C_{D}$ which proved to decrease the lift-to-drag ratio noticeably, however, both the $C_{T}$ and $C_{T} / C_{N}$ airfoils began to show improvement at large angles of attack due to better insensitivity to roughness caused by the free transition point being closer to the location of forced transition. The aft-located free transition points of the $C_{L} / C_{D}$ optimized airfoil showed excellent performance under free transition conditions, however, resulted in mediocre performance under forced transition conditions thereby leading to a less dominant weighted-sum performance over the other airfoils.

\section{$\mathrm{C}_{\mathrm{T}}$ Performance}

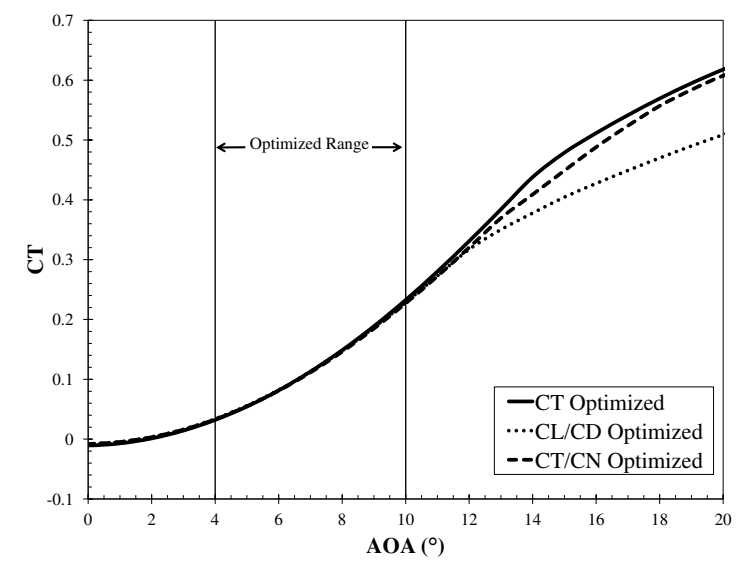

Figure 3.22: $\quad C_{T}$ of the three airfoils under free transition conditions at $R e=6 \times 10^{6}$

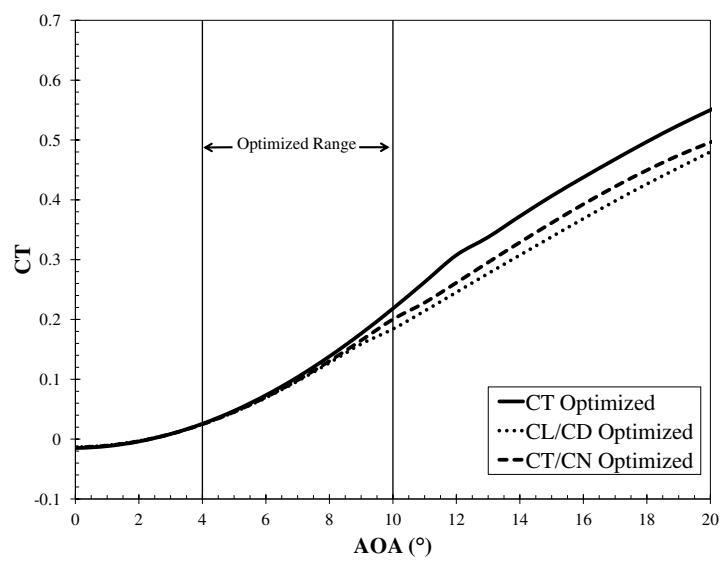

Figure 3.23: $C_{T}$ of the three airfoils under forced transition conditions at $R e=6 \times 10^{6}$ 


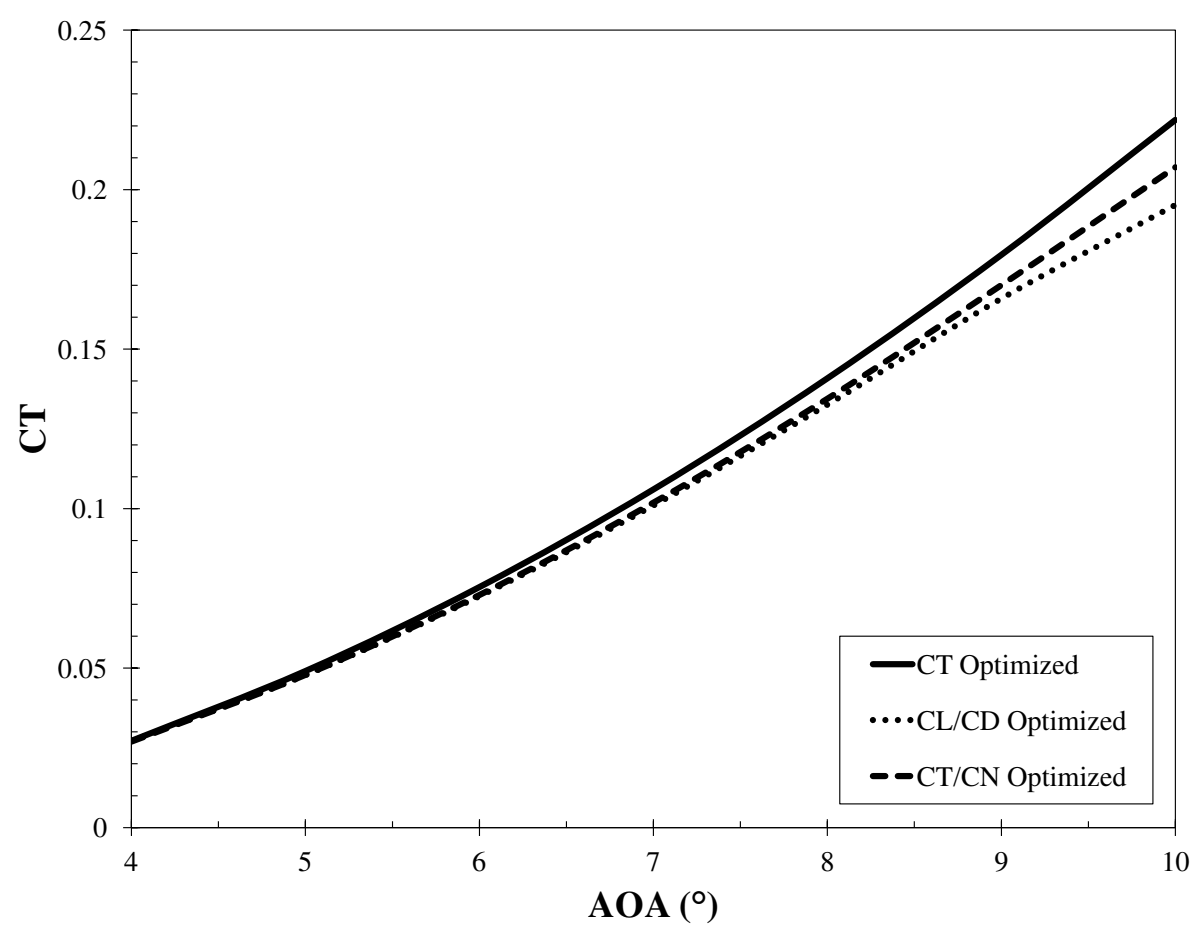

Figure 3.24: The $C_{T}$ weighted-sum ( $25 \%$ free, $75 \%$ forced transition) performance of each of the three airfoils in the $\alpha$ range

Figures 3.22-3.23 shows the free and forced transition $C_{T}$ performance of the three airfoils. In both cases, the $C_{T}$ optimized airfoil has performance that is equal to or greater than the remaining two airfoils. As shown in Figure 3.24, this undoubtedly leads to the weighted-sum performance of the $C_{T}$ optimized airfoil to exceed the remaining two airfoil's performance over the entire optimized $\alpha$ range. Its insensitivity to roughness is demonstrated by its ability to retain similar levels of lift and drag under free and forced conditions. In the calculation of $C_{T}$, the high levels of lift that were maintained at large angles of attack proved to be dominant over the relatively high levels of $C_{D}$ indicating that an airfoil which exhibits both high lift and high drag may produce more torque than airfoils which have a high lift-to-drag ratio. Under forced transition, the $C_{T} / C_{N}$ optimized airfoil was able to maintain reasonably high levels of lift, without too large of an increase in drag which resulted in it outperforming the $C_{L} / C_{D}$ optimized airfoil which exhibits poor insensitivity to roughness. 


\section{$\mathrm{C}_{\mathbf{T}} / \mathrm{C}_{\mathrm{N}}$ Performance}

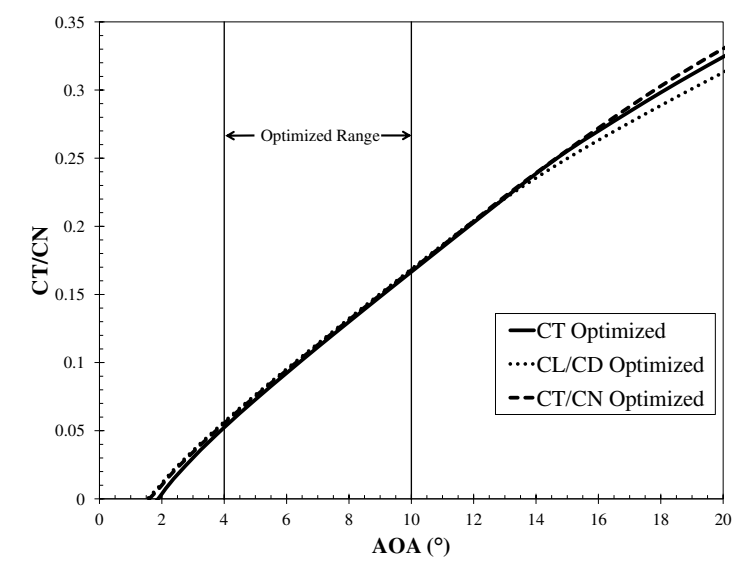

Figure 3.25: $C_{T} / C_{N}$ of the three airfoils under free transition conditions at $R e=6 \times 10^{6}$

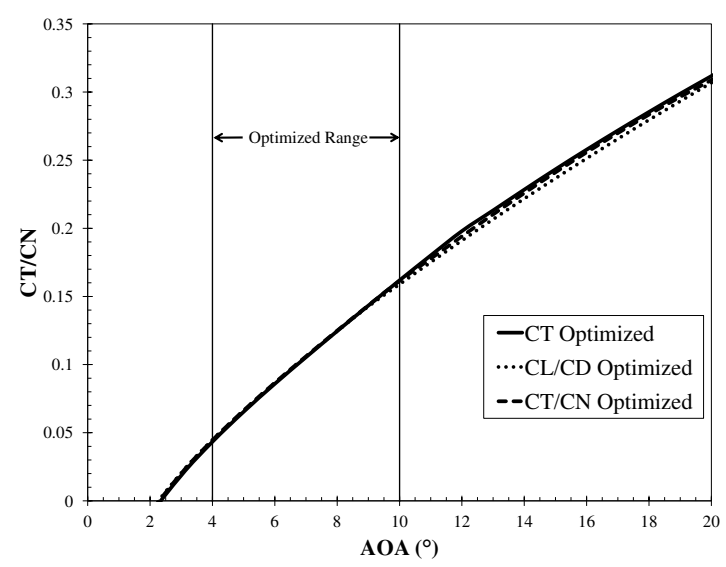

Figure 3.26: $C_{T} / C_{N}$ of the three airfoils under forced transition conditions at $R e=6 \times 10^{6}$

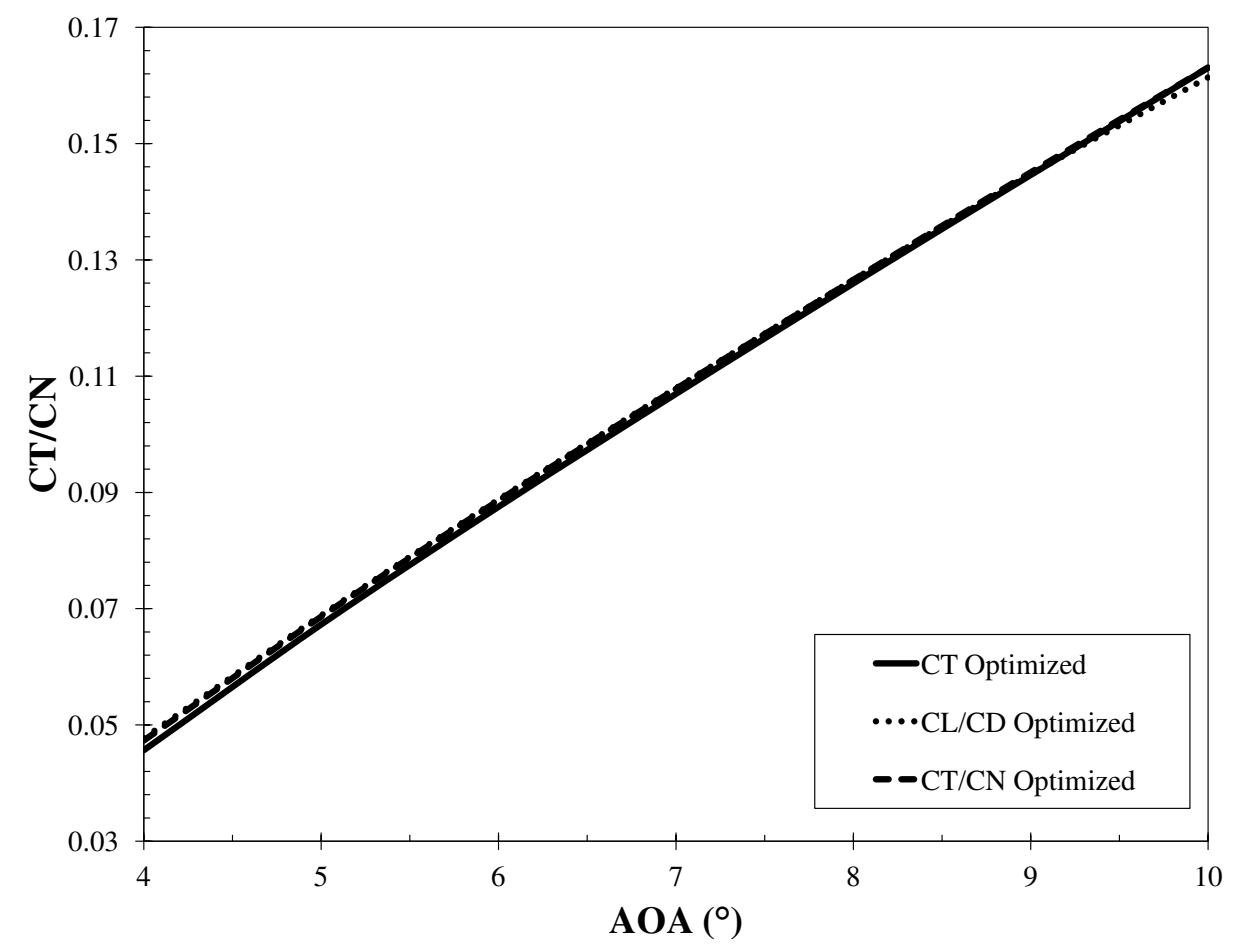

Figure 3.27: The $C_{T} / C_{N}$ weighted-sum ( $25 \%$ free, $75 \%$ forced transition) performance of each of the three airfoils in the $\alpha$ range 
Figure 3.25 and 3.26 show the free and forced transition $C_{T} / C_{N}$ performance of all three airfoils. Figure 3.27 shows the weighted-sum $C_{T} / C_{N}$ performance over the $\alpha$ range, and it can be shown from all three figures that the airfoils all have similar performance. Closer inspection reveals that for the majority of the optimized $\alpha$ range, the $C_{T} / C_{N}$ optimized airfoil is the best performing. In contrast to the plot of $C_{L} / C_{D}$ (Figure 3.21), both the $C_{T}$ and $C_{T} / C_{N}$ plots (Figures 3.24 and 3.27, respectively) do not exhibit a discernible drop in performance in the $\alpha$ range- that is, the weighted-sum performance for the $C_{T}$ and $C_{T} / C_{N}$ airfoils continually increases for increasing AOA. This indicates that the flow angle, in this case the angle of attack (since $\beta=0$ ), has a greater effect on the performance than an inevitable increase in drag caused by forced transition. Therefore, optimizing under the given specified constraints and conditions for $C_{T}$ or $C_{T} / C_{N}$ will produce different airfoil shapes, and in turn performance, than $C_{L} / C_{D}$ optimized airfoils.

\subsubsection{Summary of Aerodynamic Evaluator Findings}

In summary, under the specified design constraints and objectives, the $C_{T}$ optimized airfoil favors superior roughness insensitivity at the expense of increased drag values at low angles of attack. Its high lift performance provides high levels of torque, which is particularly important in the inboard regions of the blade where the moment arm is rather short, and allows for a reduction in chord length thereby resulting in a more slender blade design. The $C_{L} / C_{D}$ airfoil favors low levels of drag, which aids in producing high lift-to-drag ratios, at the expense of poor roughness insensitivity. The $C_{T} / C_{N}$ optimized airfoil offers a balance of low drag and roughness insensitivity, and ensures that the maximum torque-to-thrust ratio is achieved such that acceptable loading characteristics are maintained. All three of the airfoils outperformed the others for their respective aerodynamic evaluator over the $\alpha$ range proving the 
effectiveness of the optimization routine.

The results in this study are dependent on the specified design constraints and conditions, however, show that differences in airfoil shape and performance are apparent depending on the aerodynamic evaluator chosen by the designer. If less consideration for contaminated conditions was introduced by using a more balanced weighted-sum of clean and tripped conditions, it is suspected that all three airfoils will begin to converge in shape. A low weighting factor towards simulated roughness will likely lead to airfoils that are more similar in shape and performance to the $C_{L} / C_{D}$ optimized airfoil obtained in this study which generally displayed the best performance under clean conditions. From other airfoil designs available in the literature, it is seen that it is indeed possible to design a high $C_{L}$ Max airfoil which is insensitive to roughness using $C_{L} / C_{D}$ as the aerodynamic evaluator, however, specific constraints related to these qualities must be imposed by the designer. Using $C_{T}$ as the aerodynamic evaluator naturally tends to produce airfoils possessing these two qualities, however, caution must be taken by the designer such that $C_{L} / C_{D}$ performance is not sacrificed too greatly. Therefore, the proper choice of constraints and objectives for a particular aerodynamic evaluator are essential in the proper design of wind turbine airfoils.

\subsection{Constraint and Objective Sensitivity}

In the optimization of wind turbine airfoils, the choice of constraints and objectives are driven by the goals of the particular wind turbine design. They must account for the desired loading, control theories, environmental conditions, manufacturing and transportation requirements, and more. Through the author's own experience, as well as through discussion with leading experts in the field, the importance and influence of 
design constraints and objectives became apparent; e.g. a particular weighting factor between free and forced transition, $C_{L}$ Max , or angle of attack range could lead to an entirely different design outcome.

Most of the available literature will state some of the design objectives quantitatively, however, many of the design features are described qualitatively using phrases such as "a weighted sum between free and forced transition conditions was used", "the $C_{L}$ Max under forced transition was constrained to be close to that of free transition", or "transition must occur near the leading-edge". Although this provides a description of the design constraints, it does not indicate the values chosen, nor the reason for the chosen value. Therefore, to better understand how the choice of constraints and objectives effects the outcome, a sensitivity analysis was conducted.

The Taguchi method implemented in this work takes advantage of orthogonal arrays to reduce the number of design evaluations required to obtain statistical data [20]. Say for a particular design there are 4 different parameters which may be varied at 4 different levels. If every possible combination was evaluated, $4^{4}=256$ experiments/runs would be necessary. Orthogonal arrays allow for each parameter to be tested against another at each level once, thereby reducing the number of evaluations necessary to 16 for this example. As can be seen in Table 3.2 [114], each parameter $(\mathrm{A}, \mathrm{B}, \mathrm{C}$, and $\mathrm{D})$ is tested at each level $(1,2,3$, and 4$)$ once while another parameter is held constant; this is true for any two chosen parameters (columns). The sensitivity of the resultant performance outcome, denoted by $\mathrm{R}$, with respect to the variation of a certain parameter can then be examined. 
Table 3.2: Sample $L_{16}$ Taguchi orthogonal array consisting of 4 parameters (A, B, $\mathrm{C}, \mathrm{D})$ at 4 different levels $(1,2,3,4)$

\begin{tabular}{|c||c|c|c|c||c|}
\hline \multicolumn{5}{|c|}{ Taguchi $L_{16}$ array } \\
\hline Run \# & $\mathrm{A}$ & $\mathrm{B}$ & $\mathrm{C}$ & $\mathrm{D}$ & $\mathrm{R}$ \\
\hline 1 & 1 & 1 & 1 & 1 & $R_{1}$ \\
2 & 1 & 2 & 2 & 2 & $R_{2}$ \\
3 & 1 & 3 & 3 & 3 & $R_{3}$ \\
4 & 1 & 4 & 4 & 4 & $R_{4}$ \\
5 & 2 & 1 & 2 & 3 & $R_{5}$ \\
6 & 2 & 2 & 1 & 4 & $R_{6}$ \\
7 & 2 & 3 & 4 & 1 & $R_{7}$ \\
8 & 2 & 4 & 3 & 2 & $R_{8}$ \\
9 & 3 & 1 & 3 & 4 & $R_{9}$ \\
10 & 3 & 2 & 4 & 3 & $R_{10}$ \\
11 & 3 & 3 & 1 & 2 & $R_{11}$ \\
12 & 3 & 4 & 2 & 1 & $R_{12}$ \\
13 & 4 & 1 & 4 & 2 & $R_{13}$ \\
14 & 4 & 2 & 3 & 1 & $R_{14}$ \\
15 & 4 & 3 & 2 & 4 & $R_{15}$ \\
16 & 4 & 4 & 1 & 3 & $R_{16}$ \\
\hline
\end{tabular}

The four different parameters chosen for the sensitivity analysis are $C_{L \text { Max }}$, $C_{L}$ ratio, $A O A_{\text {range }}$, and weight which represent the maximum value of $C_{L}$ achieved, the minimum ratio between forced and free transition $C_{L}$ values, the distance in degrees of the upper end of the $6^{\circ}$ optimization range to $C_{L}$ Max (Figure 3.28), and the weighting between free (weight) and forced (1-weight) conditions, respectively. These parameters were chosen because they are readily variable in the developed tool and have been observed to make a notable difference in previous design iteration outcomes. This sensitivity analysis was conducted for $24 \%, 30 \%$, and $36 \%$ thick airfoils. 


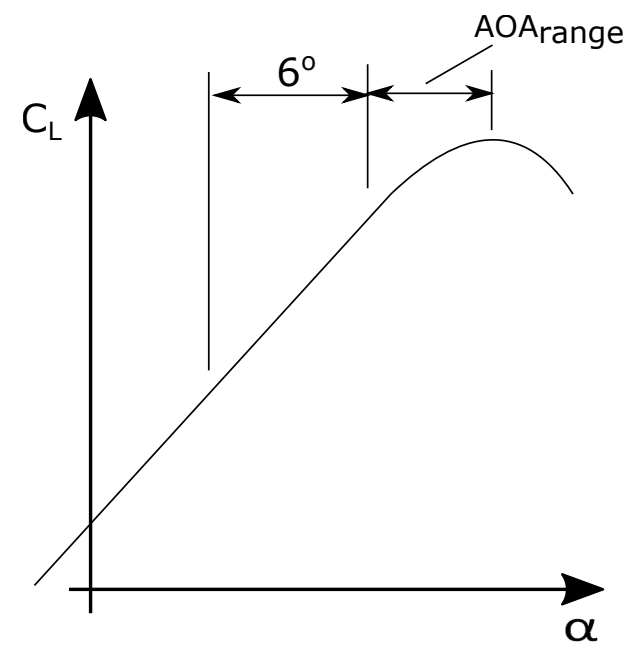

Figure 3.28: The definition of $A O A_{\text {range }}$; the distance of the upper limit of the $6^{\circ}$ optimization range to $C_{L}$ Max

In order to select appropriate levels for each of these parameters, several other airfoils from the FFA, DU, and NREL families were examined [72,73,75]. General trends, highlighted by a 2nd-order polynomial trendline in Figure 3.29, for $C_{L}$ Max, and $C_{L}$ ratio with respect to thickness were established. It can be seen that in general, an increase in airfoil thickness comes with a slight increase in $C_{L}$ Max and a notable decrease in $C_{L \text { ratio. }}$ For a given airfoil thickness, an approximate range in values of $C_{L}$ Max , and $C_{L \text { ratio }}$ was obtained and used for the Taguchi sensitivity analysis; these levels are shown in Table 3.3.
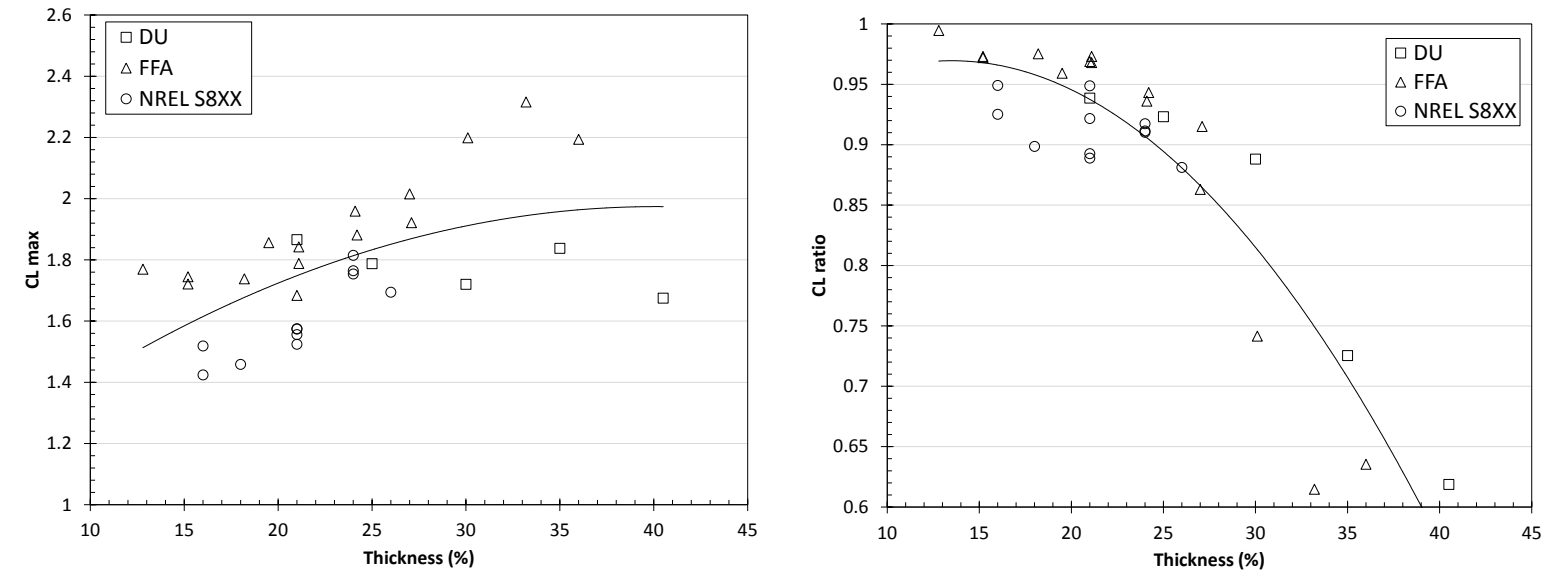

Figure 3.29: The $C_{L}$ Max (left) and $C_{L}$ ratio (right) as calculated by XFOIL at $R e=6 \times 10^{6}$ under free transition of other wind turbine specific airfoils 
Table 3.3: Parameters and associated levels for $24 \%, 30 \%$ and $36 \%$ thick airfoils

\begin{tabular}{|l||c|c|c|c|}
\hline \multicolumn{5}{|c|}{$24 \%$ thick airfoils } \\
\hline Parameter & Level 1 & Level 2 & Level 3 & Level 4 \\
\hline A- $C_{L}$ Max & 1.7 & 1.8 & 1.9 & 2.0 \\
B- $C_{L}$ ratio & 0.8875 & 0.9125 & 0.9375 & 0.9625 \\
C- AOA $A_{\text {range }}$ & 1 & 2 & 3 & 4 \\
D- weight & 0.00 & 0.25 & 0.50 & 0.75 \\
\hline
\end{tabular}

\begin{tabular}{|l||c|c|c|c|}
\hline \multicolumn{5}{|c|}{$30 \%$ thick airfoils } \\
\hline Parameter & Level 1 & Level 2 & Level 3 & Level 4 \\
\hline A- $C_{L}$ Max & 1.7 & 1.8 & 1.9 & 2.0 \\
B- $C_{L}$ ratio & 0.825 & 0.850 & 0.875 & 0.900 \\
C- AOA $A_{\text {range }}$ & 1 & 2 & 3 & 4 \\
D- weight & 0.00 & 0.25 & 0.50 & 0.75 \\
\hline
\end{tabular}

\begin{tabular}{|l||c|c|c|c|}
\hline \multicolumn{5}{|c|}{$36 \%$ thick airfoils } \\
\hline Parameter & Level 1 & Level 2 & Level 3 & Level 4 \\
\hline A- $C_{L}$ Max & 1.8 & 1.9 & 2.0 & 2.1 \\
B- $C_{L}$ ratio & 0.710 & 0.735 & 0.760 & 0.785 \\
C- AOA $A_{\text {range }}$ & 1 & 2 & 3 & 4 \\
D- weight & 0.00 & 0.25 & 0.50 & 0.75 \\
\hline
\end{tabular}

The average value of performance for each parameter, at each particular level, was calculated and normalized with respect to the overall average performance value found during the study. For example, referring to Table 3.2, Parameter $\mathrm{C}$ had a level of 3 during Runs \# 3, 8, 9 and 14. Therefore, the performance of these 4 runs, $R_{3}, R_{8}$, $R_{9}, R_{14}$, were averaged and normalized with respect to the overall average. In this study, for the 4 separate parameters, both the free transition and forced transition $C_{L} / C_{D}$ performance over the $6^{\circ}$ optimization range were simultaneously evaluated. Figure 3.30 summarizes the findings of this study. The normalized airfoil performance with respect to each parameter (rows) is shown for each airfoil thickness (columns). 


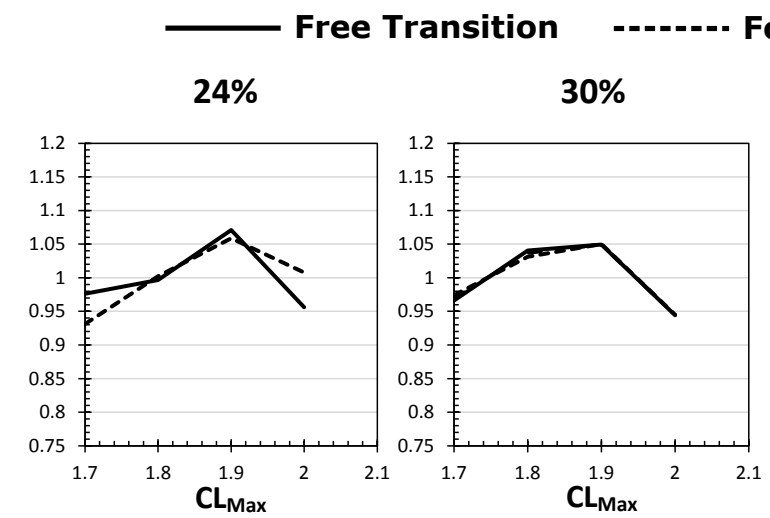

Forced Transition
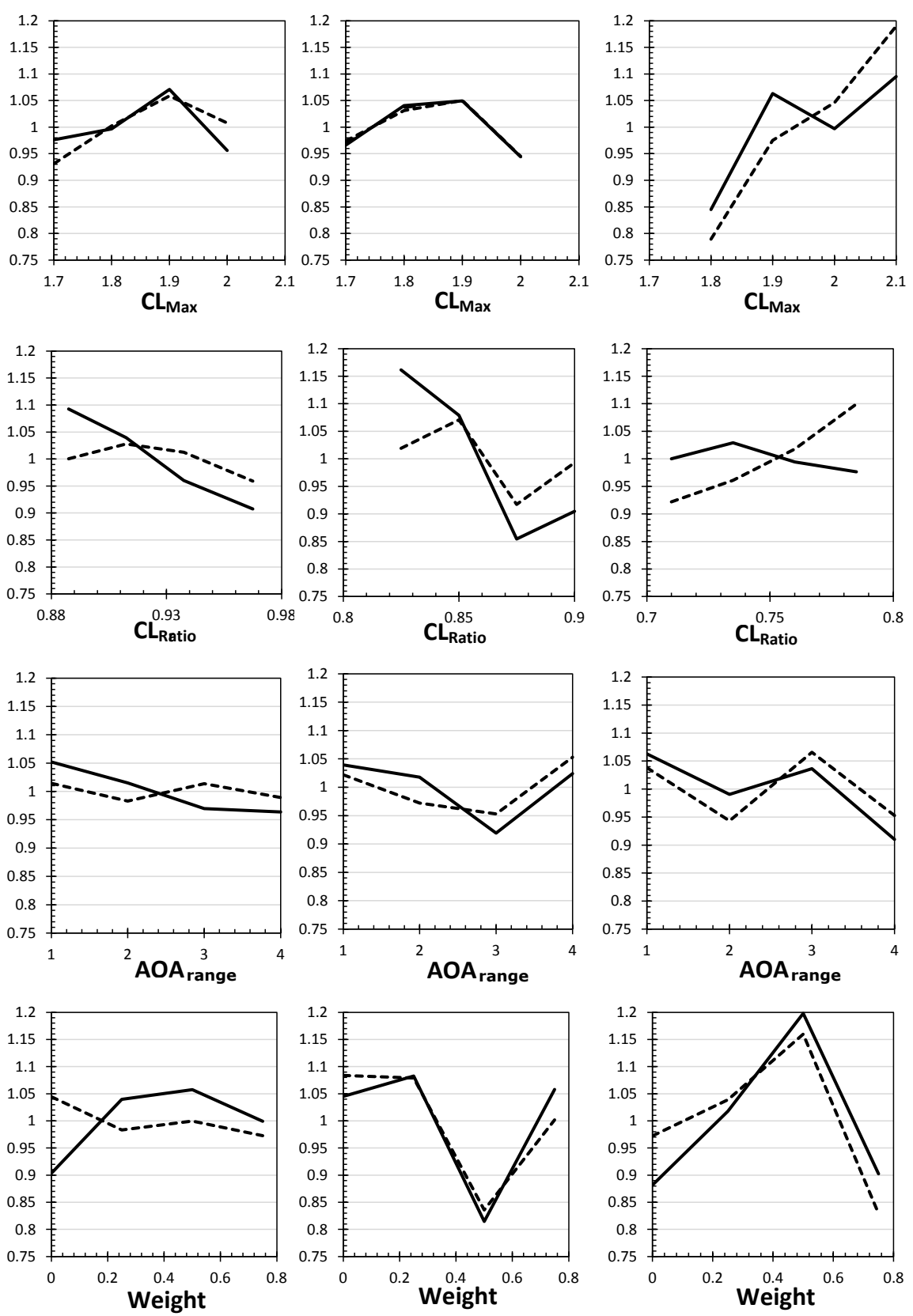

Figure 3.30: The results of the sensitivity analysis using the Taguchi method for $24 \%, 30 \%$, and $36 \%$ thick airfoils. Note that in all cases, the y-axis shows the percent change relative to the average result for each respective thickness and configuration (free or forced transition) and the $\mathrm{x}$-axis is the value for each constraint/objective 
General trends and findings include:

- The variation of performance with respect to each parameter generally increases with airfoil thickness indicating higher sensitivity to constraints and objectives for thicker airfoils

- $C_{L \text { Max }}$ and weight tend to cause the largest variations in performance

- As the $C_{L}$ ratio increases, the clean and rough performance tends to decrease

- $A O A_{\text {range }}$ has a smaller effect on the performance relative to the other parameters

- For the most part, both the free and forced transition performance followed the same general trends indicating that the parameters tested affect both with the same tendency

- One would expect that as more weight is put towards free transition conditions, the free transition performance would be increased, however, this is not necessarily the case. No clear trend in the chosen value of weight can be established that is applicable to all airfoil thicknesses tested

Although this study provides indications as to which parameters have the greatest effect on the airfoil performance outcome, unfortunately, it does not show any universally optimal settings for each parameter. However, what this study does show is that unique values of each parameter (constraint) may be necessary to ensure an optimal design. Since this analysis employs the Taguchi method which only provides a statistical estimate, perhaps a more complete analysis for which every possible combination of parameters is tested would reveal more conclusive data, however, this is very computationally expensive. 
Since weight is not a physical phenomenon related to the airfoil performance, but solely acts to influence the way in which the optimization routine interprets the design outcome, its value can be altered while maintaining specific levels of $C_{L}{ }_{M a x}, C_{L}$ ratio, and $A O A_{\text {range }}$ which may be required for the overall wind turbine design philosophy. Therefore, within a certain set of design specifications, weight may be altered to find the optimal design, without sacrificing any design constraints.

\subsection{Airfoil Family}

As found earlier, the choice of constraints and objectives will have a large impact on the shape and performance of wind turbine airfoils. These constraints and objectives are often dictated by the wind turbine design philosophy and will define specific values to be used in the optimization scheme, however, certain parameters are still left to the designer to decide on appropriate values. With this in mind an airfoil family consisting of 6 airfoils was designed spanning 21-36\% in thickness, in 3\% increments. A naming scheme, CU-W1-XX, is used where CU stands for Carleton University, the $\mathrm{W}$ stands for wind turbine airfoils, the 1 stands for the first generation, and the $\mathrm{XX}$ is the percent thickness of the airfoil.

The objective of the optimization was to maximize the $C_{L} / C_{D}$ within the AOA design range. Although other aerodynamic evaluators such as $C_{T}$, or $C_{T} / C_{N}$ could have been used, it was decided that for comparison purposes with other airfoils, $C_{L} / C_{D}$ was to be used as the aerodynamic evaluator. As will be later seen, this decision also proves that with the proper constraints, airfoils can indeed be designed to concurrently possess high lift, good roughness insensitivity, and aerodynamic efficiency, even when designed for $C_{L} / C_{D}$.

An aerodynamic constraint was used such that all airfoils have a $C_{L}$ Max $\geq 1.9$. 
Based on other airfoils available in the literature, this value is on the higher end, and allows for a reduction in chord lengths thereby resulting in a more slender blade design, which aids in reducing thrust loading on the wind turbine under parked conditions. Furthermore, since roughness insensitivity is of great concern in today's wind turbine designs, a very strict criteria for the acceptable drop in $C_{L}$ Max was enforced. Figure 3.31 shows the ratio between rough and clean (forced and free transition, respectively) maximum lift of the CU airfoils (solid markers) in comparison to other airfoil families (hollow markers). During the optimization routine, these levels of $C_{L \text { ratio }}$ were imposed as a constraint to ensure that the resultant airfoils were at least the value specified in Figure 3.31.

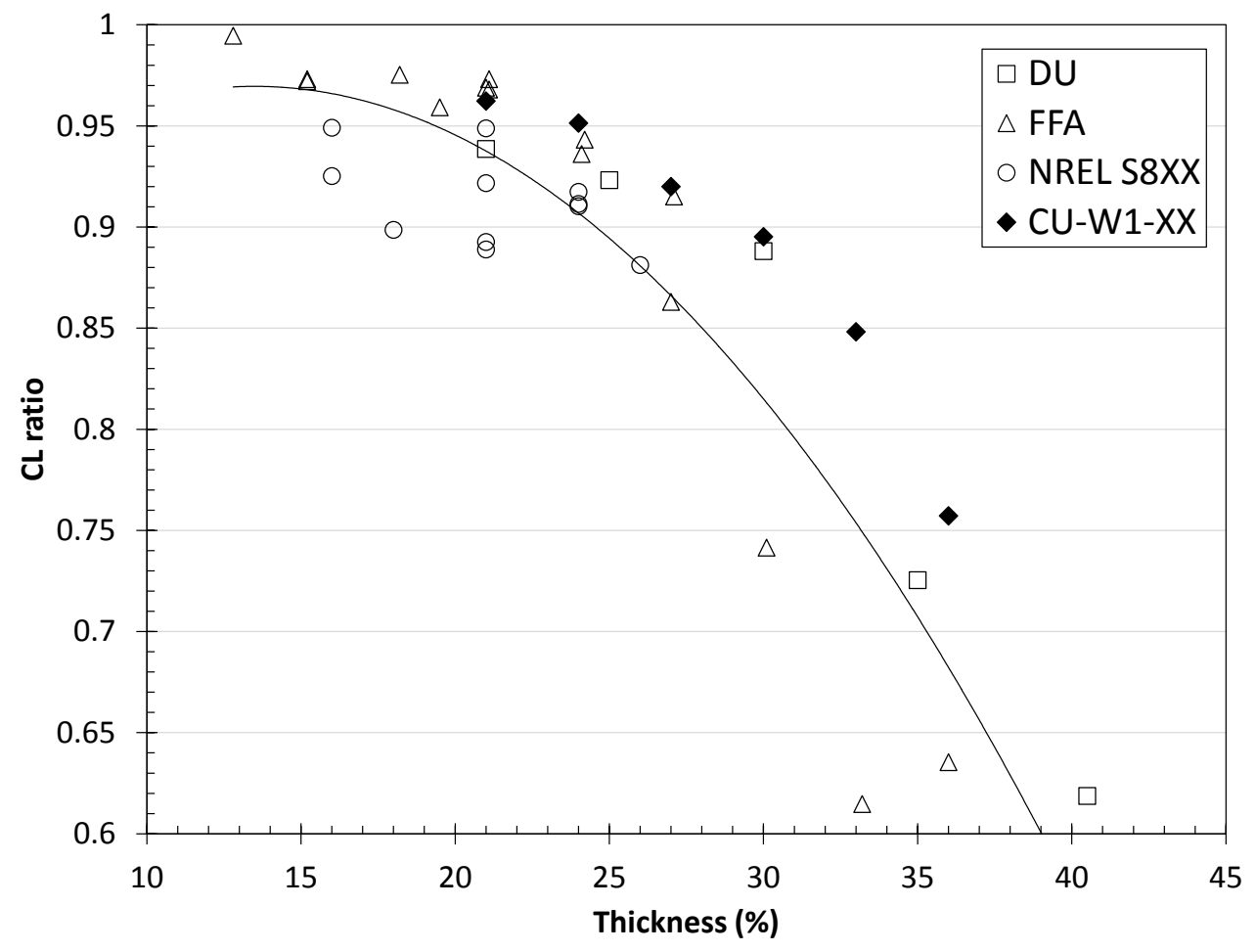

Figure 3.31: The ratio of $C_{L}$ Max in forced transition compared to free transition for the $\mathrm{CU}$ airfoils in comparison to others 
The bounds for the Bézier control points were altered depending on the specified airfoil thickness such that a more focused design space was explored, and were obtained through a combination of trial-and-error, fitting Bézier curves to existing airfoils, and interpolation/extrapolation of the bounds of other thicknesses. The generated airfoil shapes were examined to ensure that their thickness fell within the specified value $\pm 0.1 \%$. A geometric constraint was used such that the location of maximum thickness was found to be in the $x / c=0.27: 0.32$ range. Having this constraint is essential to ensuring good geometric compatibility between airfoils, and allows the blade's structural spar to pass through the thickest part of the airfoils in a straight and consistent manner. A minimum trailing-edge thickness of $0.5 \%$ was used for manufacturing feasibility purposes, and was constrained to move symmetrically about the chord line; the trailing-edge was given freedom to take on thick trailing-edge values.

With constraints for $C_{L}$ Max , and $C_{L}$ ratio set at their specified levels, single objective aerodynamic optimization (to maximize $C_{L} / C_{D}$ ) runs were performed to determine the ideal weight between free and forced transition, and the ideal $A O A_{\text {range }}$, defined as the distance in degrees from the upper limit of the 6 degree optimization range to the AOA at which $C_{L \text { Max }}$ occurs (see Figure 3.28, Section 3.5). Four separate runs were performed, each with a unique combination of weight $=0.25,0.50$ and $A O A_{\text {range }}=2,4^{\circ}$; each of these runs were examined against one another, as well as to other airfoils, to determine which offered the best performance. In almost all cases, $A O A_{\text {range }}=4^{\circ}$ provided the best performance as it corresponds to the region in which maximum $C_{L} / C_{D}$ occurs. In the few cases where it did not, a value of $4^{\circ}$ was still implemented such that a consistent design methodology was used; this may aid in ensuring a smooth geometric twist distribution along the span of the blade. For airfoils with a thickness $\geq 30 \%$, weight $=0.25$ was established to provide superior 
results, whereas for thicknesses $<30 \%$, weight $=0.50$ was found to be ideal.

The hypothesized reason for this is that for thicker airfoil sections, more attention must be paid to forced transition conditions since they are more prone to early transition and separation. By putting more attention onto forced transition conditions, a thicker trailing-edge emerges which helps to reduce the airfoil's sensitivity to roughness, while having a relatively small impact on drag values (even under free transition) since the thick airfoils already possess rather high values of drag. For the thinner airfoils, if more attention is put onto forced transition conditions, a thick trailing-edge may similarly emerge. Although this is beneficial for forced transition conditions, the corresponding increase in drag that comes with the larger trailing-edge thickness is much more detrimental to the thinner, relatively lower drag, airfoil sections. In other words, the thinner airfoil's free transition (clean) performance may be sacrificed for marginally better forced transition (rough) performance if too much weight is put towards forced transition. Therefore a more balanced combination $($ weight $=0.50)$ for thinner sections was found to be ideal.

The best performing airfoil from the single-objective optimization was then used to seed the multi-objective optimization in which structural performance was taken into consideration. This ensures that the optimization immediately starts with an airfoil that meets all constraints, and also ensures that the optimization will effectively search the extremes of the design space (i.e. the region in which the aerodynamically top performing airfoils lie). The airfoil from the resulting Pareto front with comparable $I_{X X}$ to existing airfoils of the same thickness was used for the airfoil family. The resulting airfoil family can be seen in Figure 3.32.

All of the CU-W1-XX airfoils have good geometric compatibility, with their maximum thicknesses located between $x / c=0.27: 0.29$. Furthermore, the different 


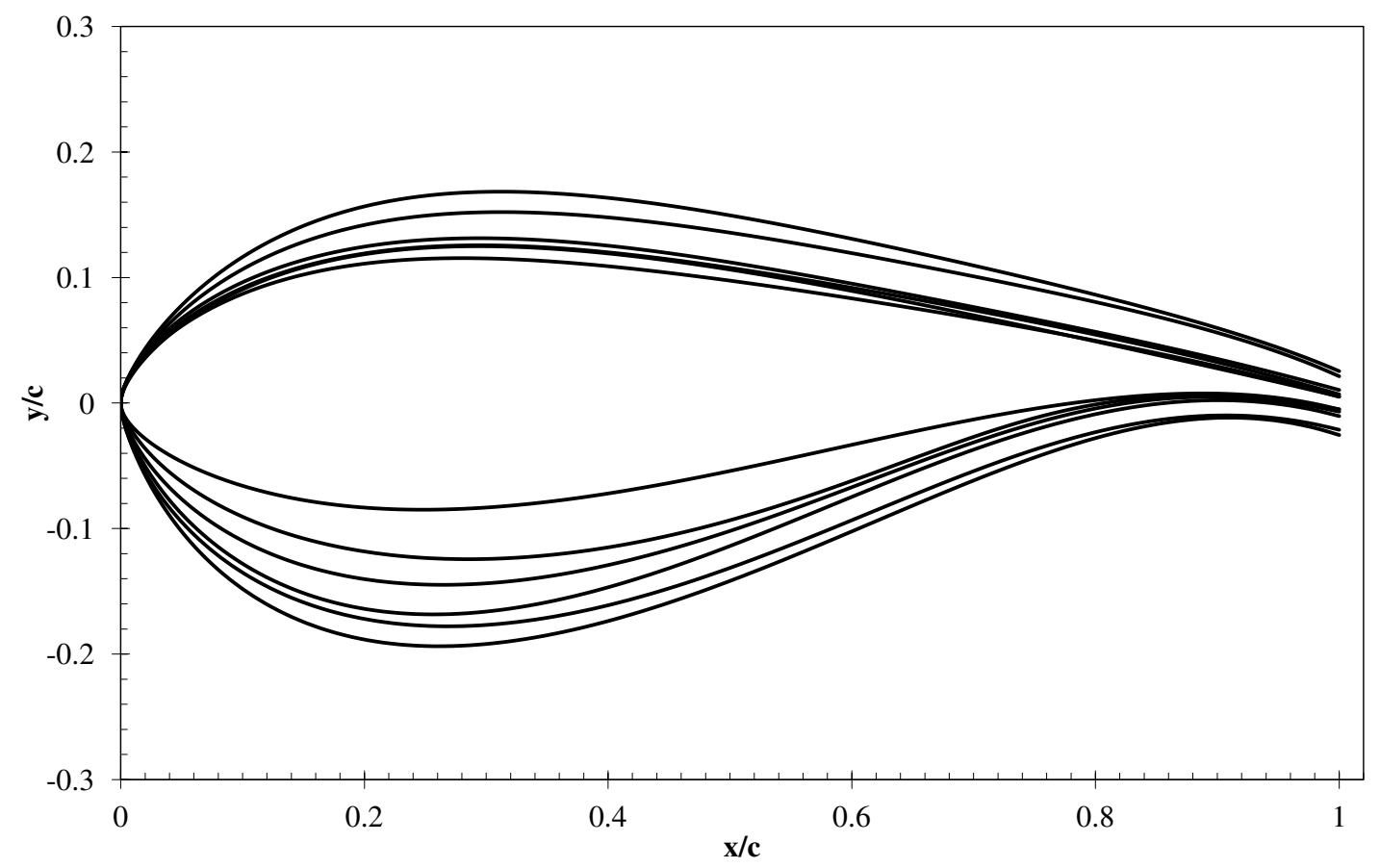

Figure 3.32: The CU-W1-XX airfoil family consisting of 6 airfoils, $21-36 \%$ in thickness

thickness airfoils show smooth changes in curvature, particularly in the leading-edge area. As shown in Figure 3.33, in all cases, superior or comparable $C_{L}$ ratio and $C_{L}$ Max were achieved when compared to other airfoils. This not only allows for better performance under contaminated conditions, but also ensures that a more slender blade design can be used. Additionally, when examining the average $C_{L} / C_{D}$ over the $6^{\circ}$ optimization range (see Figure 3.34), under free transition conditions, all of the CUW1-XX airfoils exhibit comparable, or superior performance relative to other airfoils. In forced transition conditions, the average $C_{L} / C_{D}$ performance of the CU-W1-XX family is shown to be superior compared to all other airfoils, thereby demonstrating their excellent roughness insensitivity that comes primarily due to the strict $C_{L}$ ratio constraints imposed during the design. 

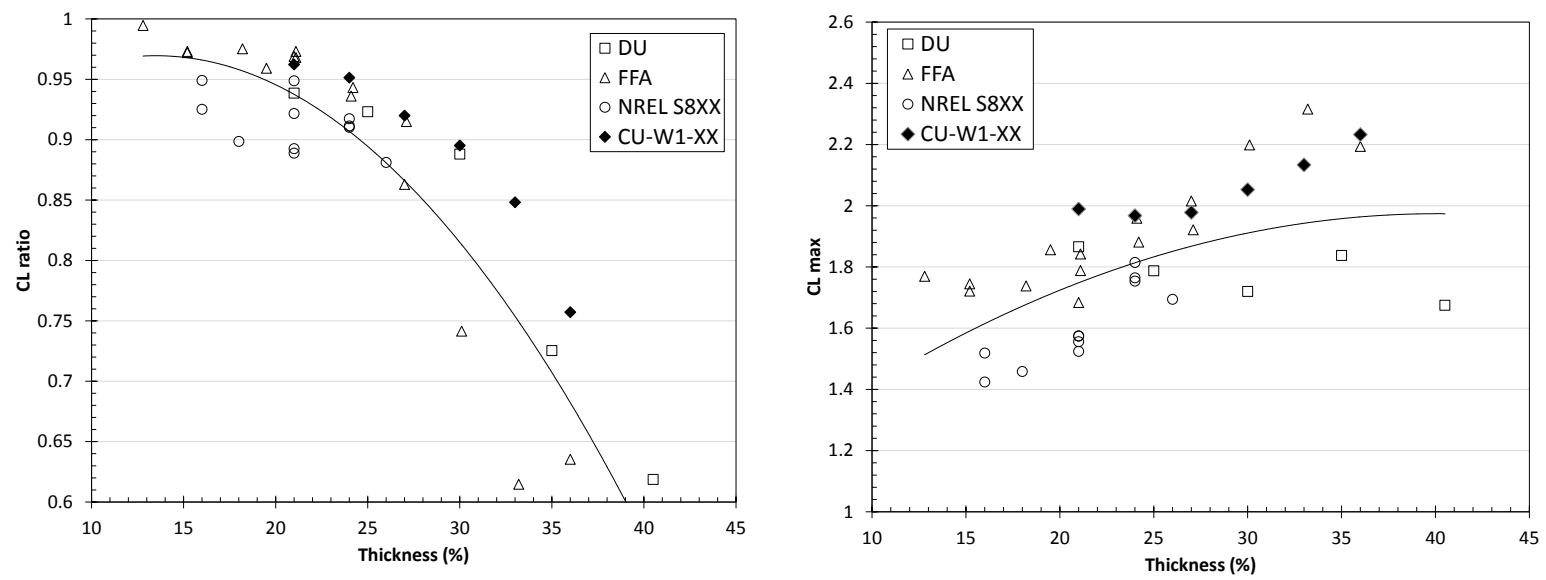

Figure 3.33: The $C_{L \text { ratio }}$ (left) and $C_{L \text { Max }}$ (right) as calculated by XFOIL at $R e=6 \times 10^{6}$ of the CU-W1-XX airfoils in comparison to other wind turbine specific airfoils
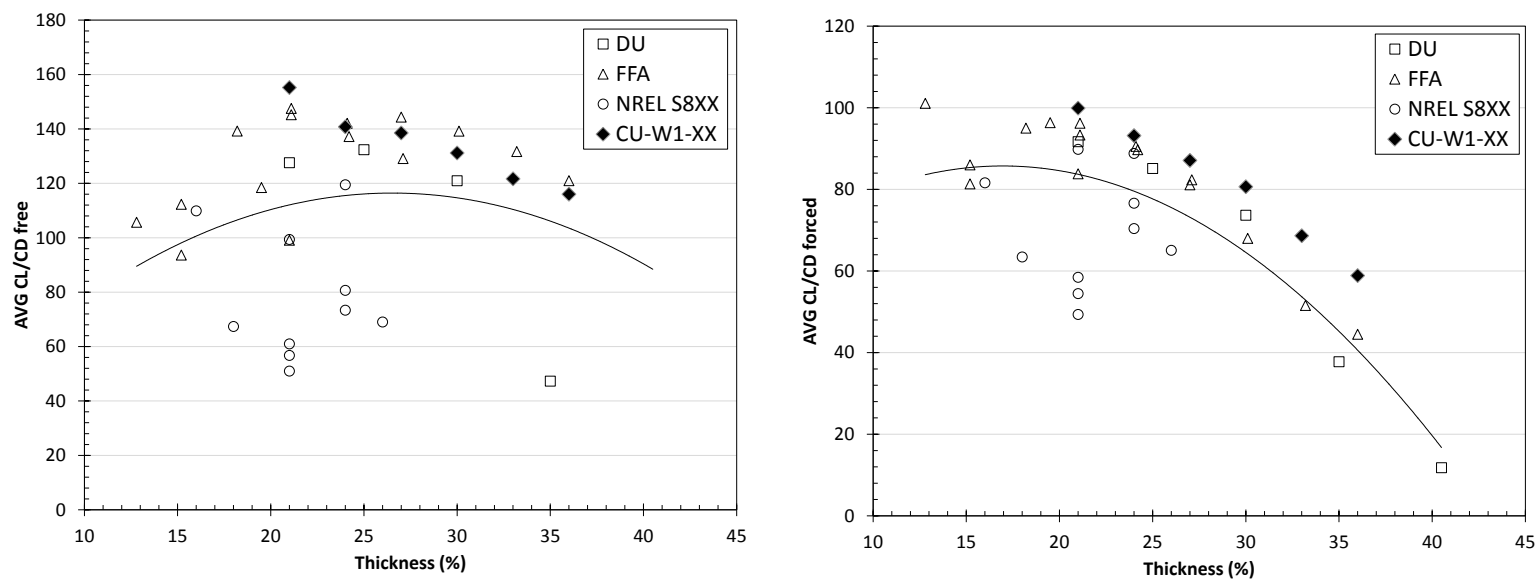

Figure 3.34: The average $C_{L} / C_{D}$ over the $6^{\circ}$ optimized range under free (left) and forced (right) transition conditions as calculated by XFOIL at $R e=6 \times 10^{6}$ of the CU-W1-XX airfoils in comparison to other wind turbine specific airfoils 
Throughout the airfoil family design process, achieving specific airfoil characteristics was found to be highly dependent on careful choice of design objectives and constraints. Oftentimes, the threshold at which certain constraints (e.g. $C_{L_{\text {ratio }}}$ ) must be set at, had to be found through trial-and-error in combination with observing the characteristics of other state-of-the-art designs. Through this process, realistic values and trends with respect to airfoil thickness, which can be used as starting-points in future designs, were acquired. In the design of an airfoil, there is not a single design which will satisfy all desirable characteristics to the fullest degree, and oftentimes trade-offs must be made, such as slightly sacrificing the $C_{L} / C_{D}$ performance under free transition conditions in exchange for superior forced transition performance. As an airfoil designer, the goal is therefore to ensure that all desirable characteristics are achieved by implementing appropriate objectives and constraints in the design process, while still taking into consideration performance trade-offs. In the design of the CU-W1-XX airfoil family, the use of unique constraints for every airfoil thickness, highlighted this point. 


\section{Chapter 4}

\section{Experimental Characterization of Airfoils}

Although XFOIL provides rather accurate aerodynamic performance predictions of 2D airfoil sections, it does have its short-comings. It has been found to under-predict drag, and over-predict maximum and post-stall lift [60-62,99,115,116]. Improvements to the numerical stability and prediction of XFOIL, particularly for thicker airfoils, and in post-stall regions, have been implemented in RFOIL. Additionally, RFOIL also implements techniques to account for the radial component of flow commonly found in the inboard region of the blade which acts to energize the boundary layer, thereby delaying stall [115]. With these radial, or rotational, effects in mind, airfoils for the inner region of the blade can be designed appropriately. Unfortunately, due to licensing restrictions, the RFOIL program suitable for optimization routines was not attainable for this work and therefore, XFOIL was used.

The thick relative thickness and flatback trailing-edges of the airfoils designed in this work, along with the fact that XFOIL was used to predict their respective performances, demands the need for experimental tests to be performed. Furthermore, the wind tunnel verification of any new airfoil design is considered to be good practice, and oftentimes a necessity in the design process. The $2 \mathrm{D}$ aerodynamic performance of 
a validation airfoil, as well as a select airfoil from this work was evaluated experimentally with a newly developed testing set-up in the Low Speed Wind Tunnel located in Mackenzie Building, Room 3224 at Carleton University.

\subsection{Equipment and Procedure}

The following sections will describe in detail the wind tunnel facility, the equipment used, the airfoil models, as well as the experimental procedures used to experimentally evaluate the aerodynamic performance of the airfoils. A schematic of the full airfoil testing set-up can be seen in Figure 4.1.

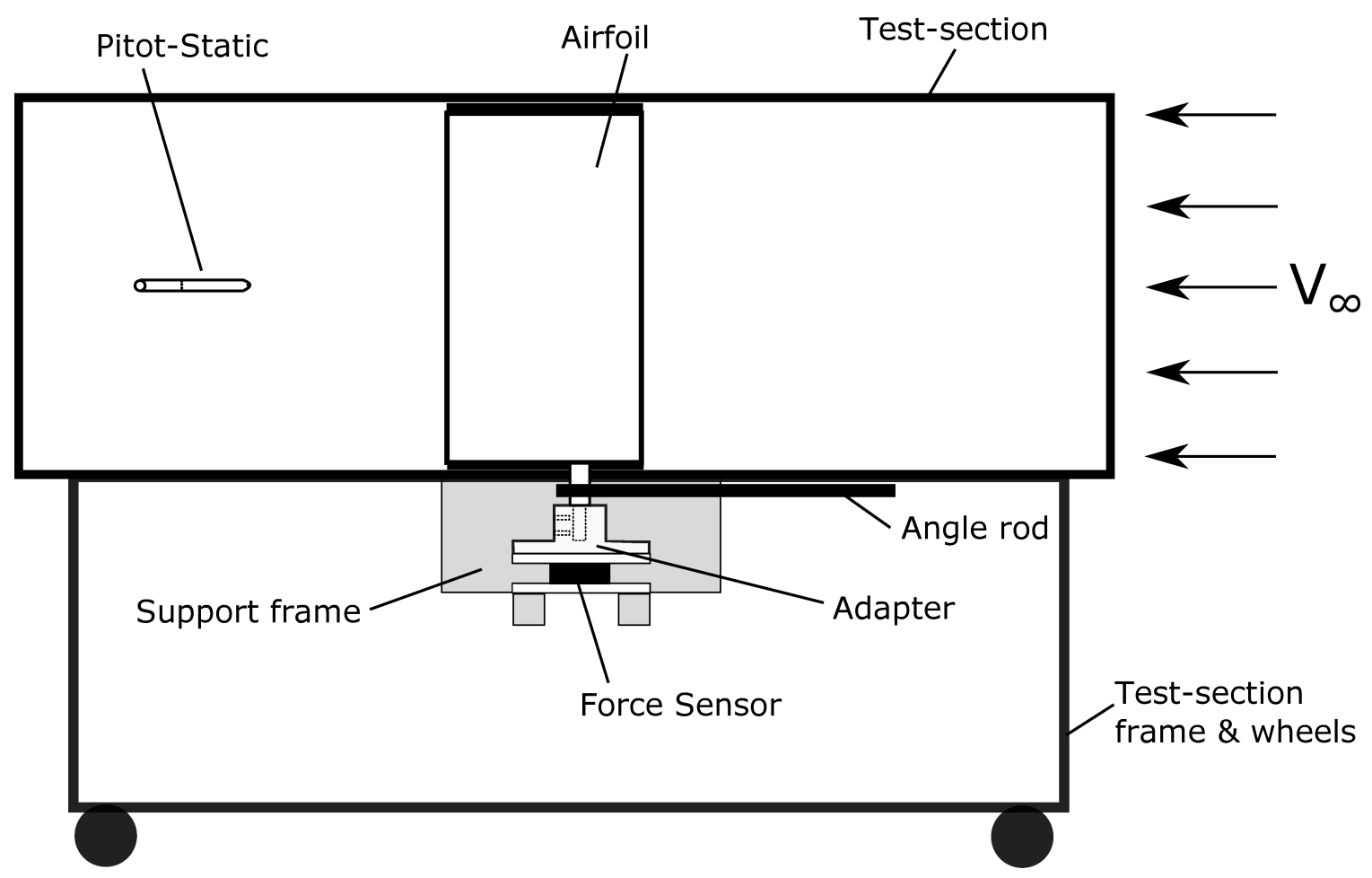

Figure 4.1: A schematic of the airfoil testing set-up in ME3224 at Carleton University. Note that schematic is not to scale. 


\subsubsection{Wind Tunnel Facility and Equipment}

\section{Wind Tunnel}

The return-circuit Low Speed Wind Tunnel in Mackenzie Building, room 3224 at Carleton University was used for all tests. Situated after the 12:1 contraction is a $1.83 \mathrm{~m}(6 \mathrm{ft})$ long test section, with a width of $0.762 \mathrm{~m} \mathrm{(30} \mathrm{in)} \mathrm{and} \mathrm{a} \mathrm{height} \mathrm{of} 0.508$ m (20 in). Centre-line velocities up to about $60 \mathrm{~m} / \mathrm{s}$, as measured with a pitot-static pressure tube connected to a U-tube manometer by the current author, are possible in the test section which has good flow angularity and turbulence intensities of $\pm 0.15 \mathrm{deg}$ and about 0.1-0.5\%, respectively, as measured by El-Ramly and Roberts [117, 118]. These good flow characteristics are vital in the testing of airfoils where for e.g. high turbulence can lead to premature transition.

It should be noted that initial trials were conducted in the 2D Transverse Vortex Wind Tunnel, located in Room ME2140 at Carleton University. This tunnel is capable of velocities up to approximately $80 \mathrm{~m} / \mathrm{s}$ [119,120] allowing for higher Reynolds number testing, however, several issues were encountered which deemed it unsuitable. Firstly, the narrow cross-section meant that very low aspect ratio airfoils must be used. Suspected 3D flow originating from near the tunnel-model junction meant that the flow was not primarily $2 \mathrm{D}$ which resulted in higher than expected drag values, and lower than expected lift values. Additionally, the 0.5 inch thick Plexiglass walls were deformed inwards due the high velocities (and thus low pressures) inside the tunnel section, and oftentimes pushed against the sides of the airfoil model making accurate force measurements difficult. Lastly, little data was available on the turbulence intensities inside the tunnel. For these reasons, the testing was instead conducted in the Low Speed Wind Tunnel, as described in the preceding paragraph.

In order to determine the coefficient of lift, drag and moment acting on an airfoil, 
the forces (in each respective direction) acting on the airfoil, the density and velocity of the air passing over the airfoil, as well as the physical dimensions of the airfoil must be determined. Additionally, the angle of attack of the airfoil must be known at all times. This necessitates equipment to measure each of the aforementioned variables.

\section{Force Sensor}

To measure the forces acting on the airfoil, a factory-calibrated ATI Delta SI-66060 6-component force transducer was used. The force sensor is mounted to a rigid support frame which is attached to the test-section frame. This force sensor is capable of measuring forces in the $\mathrm{X}$, and $\mathrm{Y}$ direction of $660 \mathrm{~N}$, in the Z direction of 1980 $\mathrm{N}$ and torques about the $\mathrm{X}, \mathrm{Y}$, and $\mathrm{Z}$ axes of $60 \mathrm{Nm}$. In the directions necessary for the characterization of the airfoil performance (X, Y, and torque about Z), the sensor has an accuracy of $1.5 \%$ of full-scale values $( \pm 9.9 \mathrm{~N}$ for forces, $\pm 0.9 \mathrm{Nm}$ for torque) with a resolution of $1 / 8 \mathrm{~N}$ and $5 / 1333 \mathrm{Nm}$. Full specifications can be found in [121]. Ideally, either a 6-component force sensor with customizable sensing limits and accuracy appropriate for each direction, or separate sensors for each direction of force, would be implemented. Alternatively, the integrated pressure from a line of static taps located along the outer surface defining the airfoil cross-sectional shape, can be used to determine the lift, moment and pressure drag forces. Generally this is the most common method, however requires the ability to accurately record the pressures from approximately 40-80 pressure taps, thus making additional equipment necessary [122].

\section{Pitot-Static Tube}

Due to the expected low magnitude of the drag forces, particularly for the attachedflow regime, a pitot-static tube was employed to accurately measure drag rather 
than the force sensor. When aligned with the flow, the $90^{\circ}$ bend pitot-static tube measures both the stagnation (total) pressure, as well as the static pressure at a given location. By connecting the total and static pressure tubes to each end of a U-tube manometer, the height difference in water can be used to calculate the air velocity. It is recommended that the measurements are taken at least 0.7 chords downstream from the trailing-edge such that the airflow has been given sufficient time to equalize to the tunnel static pressure, and that the traverse measurements are taken approximately 1 tube diameter apart [122]. The wake measurements in this work were taken 1 chord $(0.3 \mathrm{~m})$ downstream from the trailing-edge, and traversed every $1 / 4$ " (tube diameter is 3/8") for 6-9", depending on the angle of attack. Markings are made every $1 / 4$ " on the pitot-static tube and it was ensured that the tube was in-line with the flow at all times.

As will be described in a later section, the loss in velocity (momentum) in the wake behind the airfoil can be correlated to the drag force acting on the airfoil. This method was found to be much more accurate than the force sensor in the low angle of attack region where the flow was primarily attached. At higher angles of attack, the unsteadiness of the separated flow resulted in higher fluctuations in the measurements, and the inherently 3D turbulent flow makes the use of the pitot-static tube a less robust method of drag measurement; this was also found in [123].

\section{Temperature and Pressure Measurement}

In order to calculate the density of air, the temperature of the flow within the test section, as well as the atmospheric pressure must be measured. The temperature was measured using a Type $\mathrm{K}$ thermocouple inserted in the downstream section of the wind tunnel test section. The atmospheric pressure of the air was taken as reported by Environment Canada at the Ottawa International Airport [124]. The atmospheric 
pressure was monitored throughout the tests and never fluctuated by more than 0.1 $\mathrm{kPa}$ (the resolution of the data). The steadiness of the atmospheric pressure, along with the fact that the downstream end of the test section is slightly open, thereby allowing it to equalize to the same level as the atmospheric pressure, is considered to be adequately accurate for the measurements at hand.

\section{Angle of Attack Measurement}

To measure the angle of attack of the airfoil, a rod was attached to the square shaft of the airfoil (to be described in further detail later) on the underside of the test section. This stiff rod, which is about $55 \mathrm{~cm}$ long, has a channel measuring $3.175 \mathrm{~mm}$ (1/8") wide and $25 \mathrm{~mm}$ (1") long which acts as a viewfinder. A compass has been drawn on white bristol board, with angle increments every $1^{\circ}$ as measured by a Lee Valley digital protractor $\left( \pm 0.3^{\circ}\right.$ accuracy [125]), and attached to the underside of the test section with the $0^{\circ}$ marking aligned with the direction of the flow. The sketch in Figure 4.2 illustrates the angle of attack measurement technique employed in this work.

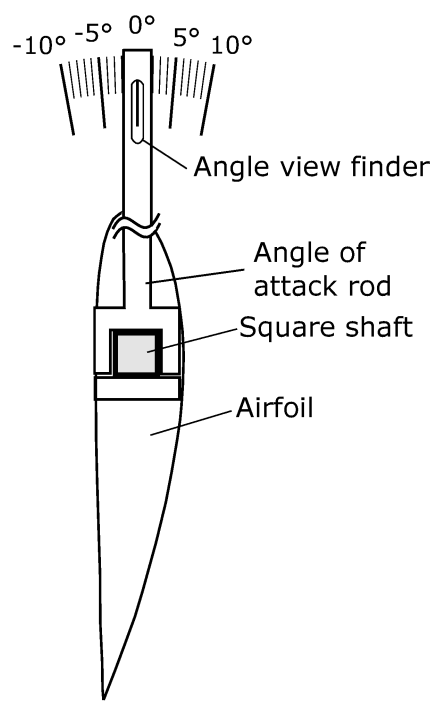

Figure 4.2: A sketch of the angle rod implemented in the experimental work. Note that the sketch is not to scale. 


\section{Force Sensor Adapter}

In order to transfer the loads acting on the airfoil to the force sensor, an adapter was manufactured out of aluminum. This adapter mounts to the force sensor on one end, and on the other, has a large collar with two set-screws. A $19 \mathrm{~mm}(0.75 ")$ square stainless steel shaft extending from the airfoil, to the outside of the test section, sits in the collar. The end of the square shaft has been machined into a cylinder which allows for the free rotation of the shaft (and therefore airfoil) in the collar such that the angle of attack can be modified. The set-screws are tightened to prevent movement of the shaft once the angle of attack has been set. The adapter and force sensor do not move, meaning that no matter the angle of attack, the forces are always in the direction of lift, drag and pitching moment. If the force sensor moved with the airfoil angle of attack, the forces would have to be resolved in their respective directions.

\subsubsection{Airfoil Model}

Several requirements were taken into consideration for the manufacture of the airfoil models including dimensional accuracy, ease of manufacture, cost, and weight. After a review of several materials and manufacturing techniques such as machining aluminum, using a mold with composite material, and additive manufacturing (3D printing), it was chosen to construct the airfoil models out of a dense prototyping foam, RenShape 5045 [126]. RenShape 5045 is a polyurethane foam with a density of approximately $300 \mathrm{~kg} / \mathrm{m}^{3}$ with easy machinability, and good edge and shape definition. Cost-wise, RenShape was approximately equivalent to aluminum, however, $9 \times$ less dense. Therefore RenShape provides a dimensionally accurate, lightweight, and easily machined material, at a reasonable cost.

The airfoils were CNC machined at the Carleton University Mechanical \& 
Aerospace machine shop to be $0.495 \mathrm{~m}$ in span, and $0.3 \mathrm{~m}$ in chord with a dimensional accuracy of approximately $\pm 0.05 \mathrm{~mm}( \pm 0.002 ")$. Each airfoil consisted of two halves machined from $50.8 \mathrm{~mm}(2 ")$ thick RenShape, with a square slot running through the center of the airfoil at the quarter-chord location for which the load transferring shaft sits. This square shaft ensures that the angle rod is oriented in line with the airfoil chord, and prevents any slippage of the shaft within the airfoil. The shaft was glued in place, with the approximately $108 \mathrm{~mm}(4.25 ")$ of the shaft extending out of one end of the airfoil. $58 \mathrm{~mm}(2.25 ")$ of this shaft was cylindrical to allow rotation within the force sensor adapter collar, while the remaining $50 \mathrm{~mm}(2$ ") was square such that the angle rod can be mounted. Double sided tape was placed between the two halves of the airfoil.

A light sanding with fine sand paper ensured that the airfoil surface was smooth. To further ensure a smooth surface, EconoKote, a lightweight shrinkable plastic covering, was applied to the outer surface. The EconoKote, typically used to cover hobby airplane wings, has a heat activated adhesive on one side, and shrinks to conform to curved surfaces [127]. The EconoKote was applied with a Coverite iron [128] at $107^{\circ} \mathrm{C}$ $\left(225^{\circ} \mathrm{F}\right)$. Adhesive-backed foam, cut to the shape of the $2 \mathrm{D}$ airfoil profile, was added to the ends of the airfoil to close the gap between the wind tunnel walls and the airfoil model. This prevents any tip vortices and ensures that the flow is primarily 2D. Three different thicknesses $\left(1 / 4 ", 1 / 8 "\right.$, and $\left.1 / 16^{\prime \prime}\right)$ of foam were used in combination to sufficiently close the gap, without causing interference with the wall which would result in the transferring of loads through the wind tunnel walls. The total thickness of foam was evaluated by trial-and-error while monitoring the forces. A photograph of the manufactured airfoil can be seen in Figure 4.3. 


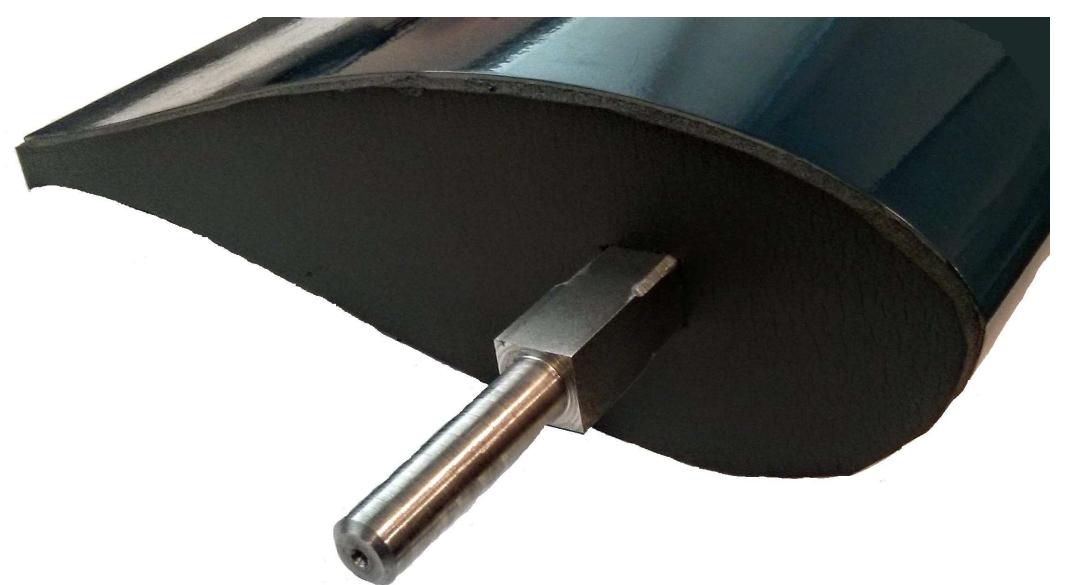

Figure 4.3: Photograph of a manufactured airfoil, showing the EconoKote covering (dark blue), the foam (black), and the airfoil shaft (metallic)

\subsubsection{Experimental Procedure}

Upon installing an airfoil in the test section, the geometric location of the leading and trailing-edge relative to the wind tunnel walls was measured using digital calipers with the airfoil set at $0^{\circ}$, as determined by the angle of attack rod. The mean of 3 measurements, taken from both sides of the wind tunnel walls, was used to calculate any geometric angular misalignment between the airfoil chord (angle rod), and the wind tunnel centerline. The geometric misalignment was accounted for by correcting the angle of attack of the airfoil during the data reduction phase. The following procedure was used for all experimental trials, in which a trial consists of a complete angle of attack sweep starting at low angles, and increasing to the max angle.

1. Three tares of the force sensor (wind-off) were performed and averaged to obtain the tare voltage readings.

2. The wind tunnel motor was set to a speed of $40 \mathrm{~Hz}$ which corresponds to approximately $40 \mathrm{~m} / \mathrm{s}$ as measured by the pitot-static tube with an empty test section. 
3. The tunnel was run for 30 minutes; sufficient time for the temperature to rise and stabilize at about $25^{\circ} \mathrm{C}$. Meanwhile the atmospheric pressure was recorded from Environment Canada and monitored at approximately 30 minute intervals throughout the test.

4. The angle of attack was set to $-4^{\circ}$ and the two set screws on the collar tightened.

5. The force sensor and thermocouple data was recorded for 2 seconds at $100 \mathrm{~Hz}$ giving 200 data points, which is similar to airfoil studies performed at the Ohio State University $[123,129]$.

6. The pitot-static tube was traversed across the wake of the airfoil for 152-229 $\mathrm{mm}(6-9 ")$ in $6.3 \mathrm{~mm}\left(1 / 4^{\prime \prime}\right)$ intervals and the data was manually read off the U-tube manometer. The range of the traverse was dependent on the angle of attack, with larger angles of attack requiring larger sweeps. All measurements started $222 \mathrm{~mm}\left(8.75^{\prime \prime}\right)$ from the wind tunnel wall on the pressure surface side of the airfoil.

7. The force sensor data was recorded once again for 2 seconds at $100 \mathrm{~Hz}$.

8. The angle of attack was increased in $2^{\circ}$ increments and steps 5-7 were repeated until an angle of attack of $+20^{\circ}$ was reached.

9. The tunnel motor was slowed down to a stop, and once sufficient time has passed to allow the airflow to stop, three tare measurements were taken. These values were compared to the initial three tares to ensure that nothing has affected the force sensor throughout the test.

The complete trial, which takes about 4 hours to complete, was repeated from start-to-finish for a total of four times on different days and times for each airfoil. 


\subsubsection{Data Reduction}

A MATLAB script was written to read the force sensor data that is output in a .csv file for each measurement. This script, which takes into account the temperature, atmospheric pressure, airfoil dimensions, and forces will resolve the data and output a file summarizing the average coefficient of lift, drag and moment at each angle of attack. It should be noted that the raw voltage readings and force data, was kept in case troubleshooting was required.
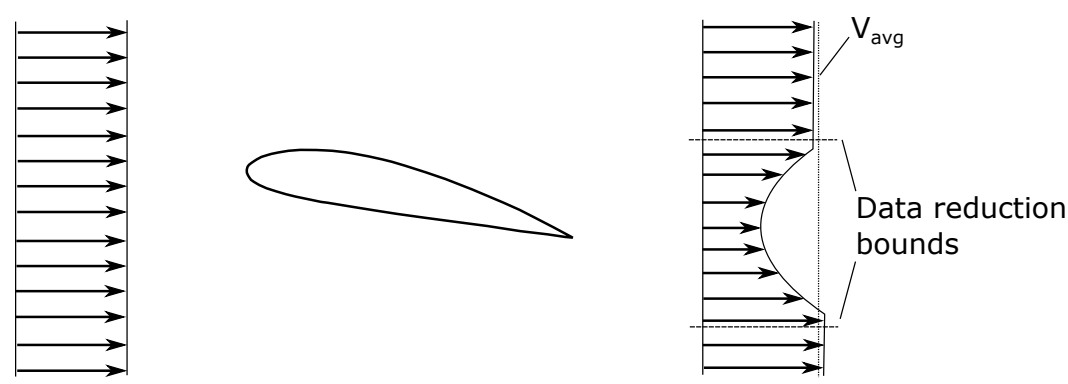

Figure 4.4: Schematic illustrating the momentum drag method. Note the bounds of the measurements used in the data reduction, as well as the averaged velocities outside the airfoil wake.

As mentioned earlier, the velocity deficit behind the airfoil can be used to calculate the drag acting on the airfoil. This loss in momentum (or velocity for a $2 \mathrm{D}$ flow) is due to both the pressure drag, and skin friction drag which extract energy from the flow. The velocity at a given point within the wake of the airfoil can be compared to that of a reference velocity, outside of the wake; it is therefore necessary to have reference velocities outside of the wake, as well as sufficient spatial resolution within the wake. At high angles of attack, due to blockage and the effect of the wake, the velocity outside of the wake on one side of the airfoil is not necessarily equal to the velocity outside the wake on the other side. The average between the two velocities was taken and the drag calculation, as derived in Equations 4.1-4.3, was performed 
from 2 data points outside the wake on one side, to 2 data points outside the wake on the other side. This methodology, similar to that implemented in [123], is illustrated in Figure 4.4 and ensures that any influence of the averaging has a minimal overall effect on the data.

$$
D=\int_{-\infty}^{\infty} \dot{m} V_{\infty}-\int_{-\infty}^{\infty} \dot{m} V
$$

where $\dot{m}=\rho V A$, however, since we are taking the measurements in a line, and not an area, we can say $\dot{m}=\rho V \partial x$ where $\partial x$ is a distance- in this case the distance between measurement points during the traverse. Rewriting Equation 4.1, and assuming constant density $(\rho)$ we get:

$$
D=\int_{-\infty}^{\infty} \rho V V_{\infty} \partial x-\int_{-\infty}^{\infty} \rho V^{2} \partial x=\rho \int_{-\infty}^{\infty} V\left(V_{\infty}-V\right) \partial x
$$

Since we are concerned with the coefficient of drag, we must divide the drag force by the dynamic pressure multiplied by the area of the airfoil, however, since we are measuring along a line, and not an area, we simply use the chord, $C$.

$$
C_{D}=\frac{\rho \int_{-\infty}^{\infty} V\left(V_{\infty}-V\right) \partial x}{\frac{1}{2} \rho V_{\infty}^{2} C}=\frac{2 \int_{-\infty}^{\infty} V\left(V_{\infty}-V\right) \partial x}{V_{\infty}^{2} C}
$$

From Equation 4.3 it can be observed that one advantage of the momentum drag method is that it does not depend on the density of the air. It can be argued that when calculating the velocity of the air (as shown in Equation 4.4), the density is a variable, however, since all calculations are relative to $V_{\infty}$, as long as the $\rho$ used is assumed to be constant, it will not affect the final result.

$$
V=\sqrt{\frac{2 \rho_{\text {water }} g H}{\rho_{\text {air }}}}
$$


where $\rho_{\text {water }}=1000 \mathrm{~kg} / \mathrm{m}^{3}, g=9.81 \mathrm{~m} / \mathrm{s}^{2}$, and $H$ is the water height difference between the static and total pressure in meters.

\subsection{Wind Tunnel Corrections}

When testing in a wind tunnel, the results obtained do not necessarily represent the aerodynamic performance in open air due to the presence of the wind tunnel walls. The wind tunnel walls confine the flow and introduce pressure gradients which alter the airflow over the model. The wind tunnel boundary corrections used in this work will be described in detail below.

\subsubsection{Solid Blockage}

The presence of a physical model within the test section results in a reduction in area that the air has to flow and according to continuity, in order to preserve the mass flow rate, a reduction in area is accompanied by an increase in velocity. Since in free-air this reduction of area is non-existent, the increased velocity in the wind tunnel is not representative of true conditions. According to Thom [130], the solid blockage, $\epsilon_{s b}$, can be calculated as:

$$
\epsilon_{s b}=\frac{K V o l}{A_{\text {sect }}^{\frac{3}{2}}}
$$

where $K=0.52$ for a wing spanning the tunnel height (as is the case in this work), $\mathrm{Vol}$ is the model volume where $0.7 \times$ thickness $\times$ chord $\times$ span is considered a good approximation for airfoils [122], and $A_{\text {sect }}$ is the test section area. 


\subsubsection{Wake Blockage}

In addition to the blockage due to the physical presence of the model, the wake which emanates from an object will further block the flow, and in turn increase the velocity around the model even more. The effect of the wake blockage, $\epsilon_{w b}$, can be calculated using Equation 4.6 which was derived by Maskell [131].

$$
\epsilon_{w b}=\frac{C / H t}{2} C_{D u}
$$

where $C$ and $H t$ are the model chord and test section height, respectively, and $C_{D u}$ is the uncorrected drag.

\subsubsection{Streamline Curvature}

The walls of a wind tunnel prevent the air from following the natural curvature around a lifting body. In turn, the straight flow in the test section mimics a higher degree of camber thereby altering the measured lift, moment and effective angle of attack acting on the airfoil. According to vortex theory, a lifting body can be represented by a single vortex. If an airfoil is represented by a vortex located at the quarter-chord location in the center of a test section, an infinite vertical row of opposite vortices must be located above and below that of the airfoil vortex to represent the walls of the tunnel. At the quarter-chord location, these vortices induce no velocity on the airfoil in the horizontal or vertical direction, however, moving along the chord of the airfoil, the horizontal components of the induced velocities cancel each other, but the vertical components induce an overall upwash on the airfoil. This upwash effectively increases the angle of attack and alters the loading along the chord thereby changing the lift and moment. [122]

The complete corrections for the angle of attack, lift, drag, and moment will be 
described in the following section. Note that these corrections are derived for a case where the vorticity is spread out along the chord of the airfoil, rather than at a single point as was described earlier. Note that the effects of buoyancy, which is the result of the thickening of boundary layers along the test section walls which reduces the effective area, and in turn increases the velocity, are not considered in this work. According to Bernoulli, the increase in velocity will result in a drop in static pressure along the length of the test section which may induce a force in the drag direction [122], however, the wake and solid blockage effects are assumed to be dominant over the buoyancy effects.

\subsubsection{Summary of Corrections}

The aforementioned wind tunnel effects are accounted for using the following equations [122]. Note that the subscript $u$ represents uncorrected data, and the factor $\sigma=\left(\pi^{2} / 48\right)(C / H t)^{2}$.

$$
\begin{gathered}
\epsilon=\epsilon_{s b}+\epsilon_{w b} \\
\alpha=\alpha_{u}+\frac{57.3 \sigma}{2 \pi}\left(C_{L u}+4 C_{M u}\right) \\
C_{L}=C_{L u}(1-\sigma-2 \epsilon) \\
C_{M}=C_{M u}(1-2 \epsilon)+\frac{\sigma C_{L}}{4} \\
C_{D}=C_{D u}\left(1-3 \epsilon_{s b}-2 \epsilon_{w b}\right)
\end{gathered}
$$




$$
R e=R e_{u}(1+\epsilon)
$$

Figures 4.5 and 4.6 show the effect of applying the aforementioned wind tunnel corrections to the measured data. The increased blockage found at high angles of attack causes the flow to increase in velocity and in turn produce larger forces. The corrections are intended to compensate for this difference in flow velocity, and generally results in a reduction of the measured forces.

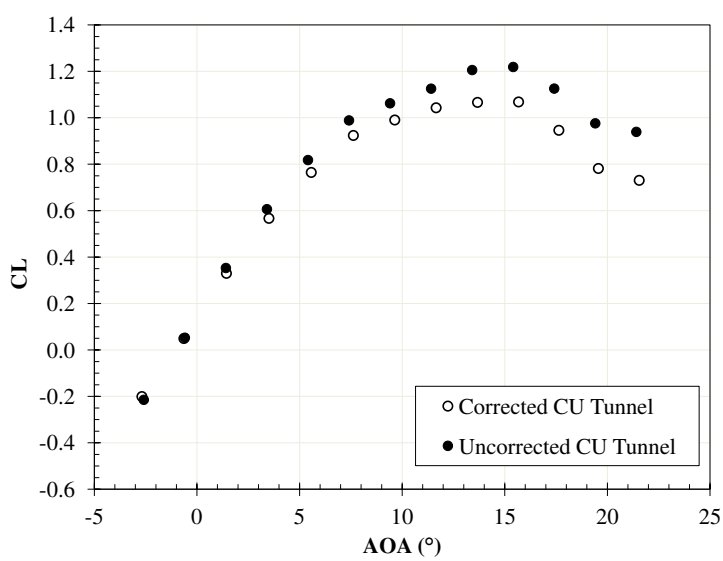

Figure 4.5: The effect of applying wind tunnel corrections to the measured lift data

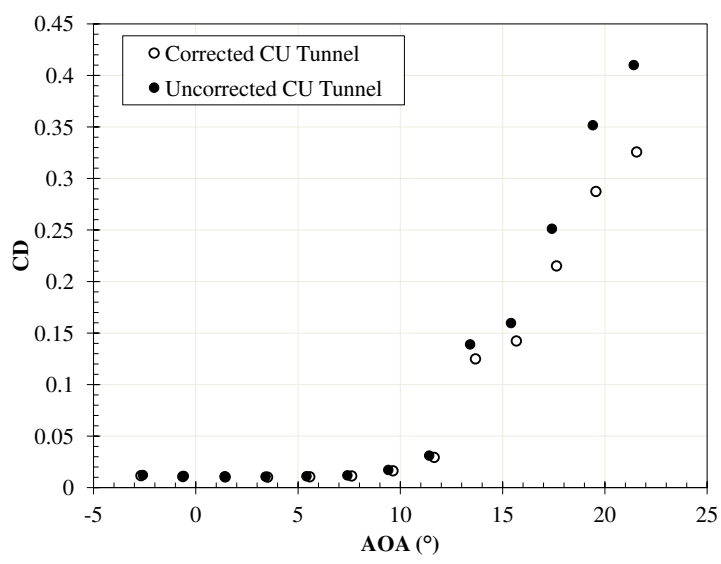

Figure 4.6: The effect of applying wind tunnel corrections to the measured drag data

\subsection{Results}

The following section will summarize the results from the experimental testing performed at Carleton University. A validation test case using the 21\% thick S809 airfoil was performed and compared to the experimental results from Ohio State University conducted as a part of the NREL Phase VI experiment [132]. Additionally, the $C_{T}$ airfoil designed in this work was experimentally tested to verify its predicted XFOIL 


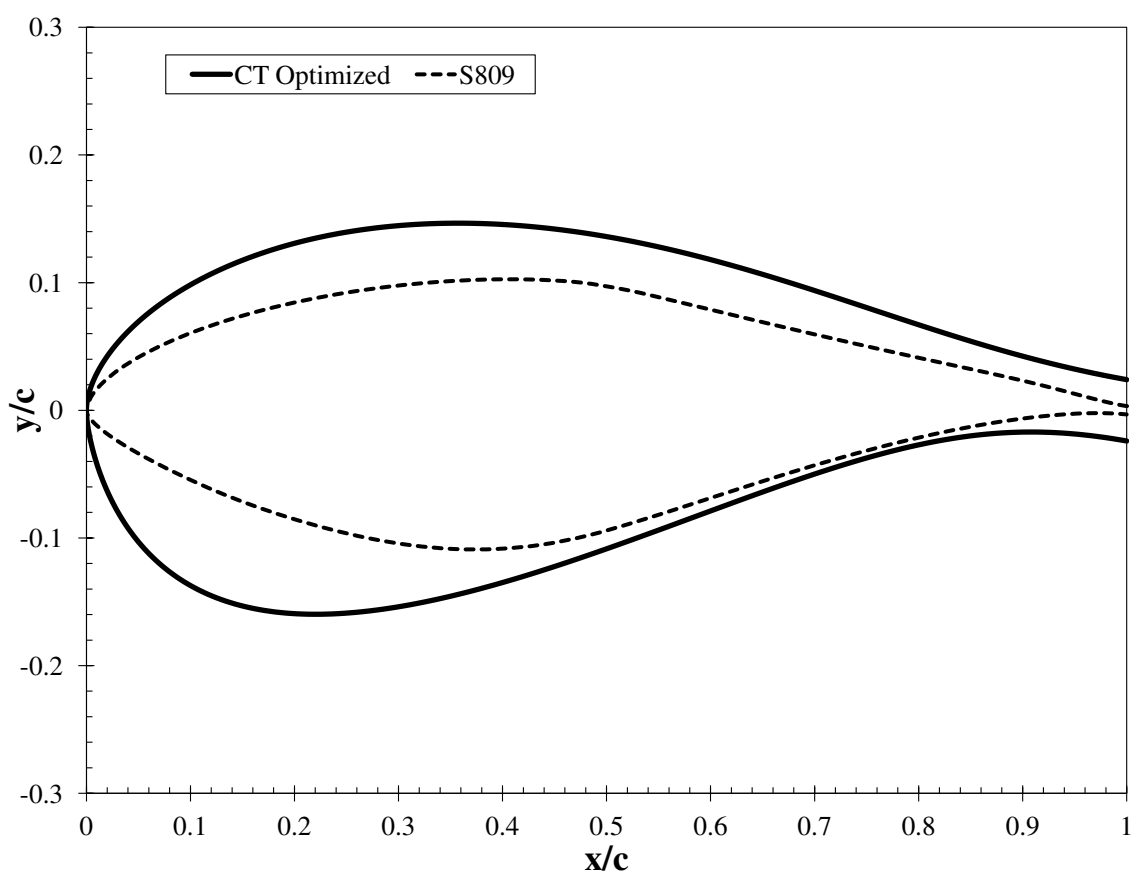

Figure 4.7: The S809 airfoil used for wind tunnel set-up and method validation purposes, as well as the $C_{T}$ airfoil developed in this work, both of which were experimentally tested at Carleton University

performance; both airfoil shapes are plotted in Figure 4.7. Finally, a summary of the uncertainty analysis will be described.

All experimental tests were conducted at approximately $R e \doteq 750,000$ (ranging from 727,000 to 770,000$)$ with a corresponding wind velocity of $40 \mathrm{~m} / \mathrm{s}(M a=0.12)$ and a chord length of $0.3 \mathrm{~m}$. This Reynolds number is within the capabilities of the wind tunnel located in ME3224 at Carleton University, and corresponds to the Reynolds number of the data available in literature used for validation purposes. Although the optimization of the airfoils was carried out at $R e=6 \times 10^{6}$, the trends of the data established at $R e \doteq 750,000$ are assumed to be consistent with higher Reynolds numbers. Figure 4.8 shows the variation of $C_{D \text { Min }}$, and $C_{L}$ Max with respect to varying Reynolds numbers as predicted by XFOIL for the CU-W1-30 airfoil. For Reynolds numbers greater than approximately $0.75 \times 10^{6}$, very little variation is found in both the lift and drag performance, and the trend is almost linear for $R e>0.75 \times$ 
$10^{6}$. Similar trends were obtained experimentally in Ref. [133] for a NACA0015 airfoil. Thus, experimental testing of the airfoils at $R e \doteq 750,000$ was deemed adequate.

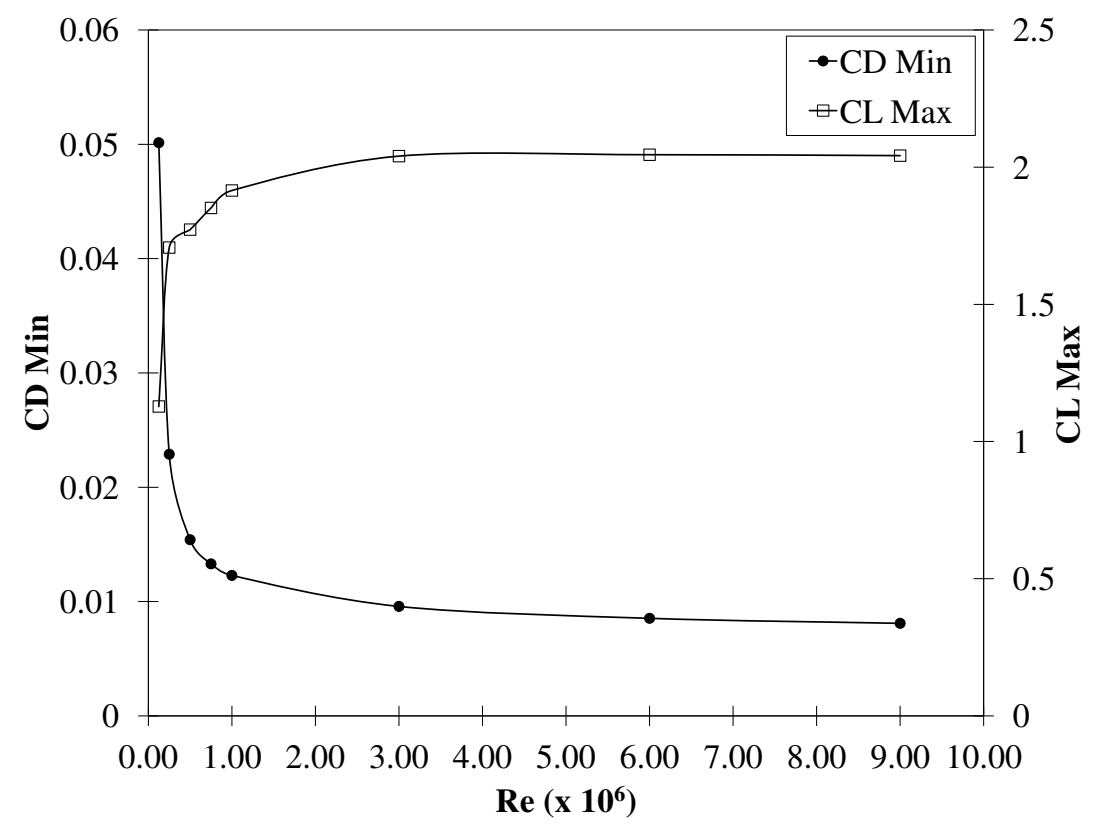

Figure 4.8: The variation of $C_{D}$ Min and $C_{L}$ Max with respect to Re as predicted by XFOIL for the CU-W1-30 airfoil

The momentum drag method was used to measure the drag up to $10^{\circ}$ (attached flow) whereas the force sensor was used for angles of attack greater than $10^{\circ}$ (separated flow). Once flow separation began, the reading of the U-tube manometer became difficult due to large fluctuations of the readings. Furthermore, the wake momentum method may not capture the inherently 3D characteristics of the separated flow if only measured along a single plane. All data presented for the Carleton University (CU) measurements have been corrected using the equations in Section 4.2.4, and are presented with error bars representing the spread in measured data. Note that the horizontal error bars for angle of attack are not shown as they are smaller than the symbols themselves. All comparisons to XFOIL have been made at $R e=750,000$, $M a=0.1$ and $N=9$. 


\subsubsection{S809 Airfoil Validation}

Since the experimental set-up and methods used in this work have never been used in this particular wind tunnel before, it is useful to repeat an existing experiment found in the literature to verify its performance. The S809 airfoil, designed at NREL, is intended for low Reynolds numbers $\left(R e \leq 2 \times 10^{6}\right)$ and has a moderate thickness of $21 \%$ [72]. Its design is intended for stall-regulated horizontal axis wind turbines with blade lengths between 10-15 m. For these reasons, it was used in the extensive NREL Phase VI experiment in which a $10 \mathrm{~m}$ diameter wind turbine was experimentally tested in the NASA Ames $20 \times 30 \mathrm{~m}$ wind tunnel. As part of the experiment, the $2 \mathrm{D}$ characteristics of the S809 airfoil were experimentally determined by Delft University and Ohio State University (OSU) at a variety of Reynolds numbers.

Since this airfoil has moderate thickness, and a thin trailing-edge, XFOIL should be able to predict its performance rather well. Additionally, the availability of published data for this airfoil make it an ideal validation test case. The S809 airfoil coordinates were taken from [132], however, for manufacturing purposes, the trailingedge was modified to be $0.66 \%$ thick by evenly adding thickness to the top and bottom surfaces over the aft $40 \%$ of the airfoil. A nominally sharp trailing-edge thickness of about $0.5 \%$ is common for the manufacture of wind turbine airfoils [39]. The XFOIL results used for comparison took the modified trailing-edge thickness into account.

Figures 4.9-4.11 show the coefficient of lift, drag, and lift-to-drag ratio for the S809 airfoil tested at Carleton University. It should be noted that the OSU data has been corrected by $-0.5^{\circ}$ due to a hypothesized angle of attack misalignment [75]. Examining the coefficient of lift in Figure 4.9, it can be observed that in the linear, attached flow region of the curve there is rather good agreement between the OSU and the CU data, whereas at higher angles of attack, there is a slight discrepancy. The 


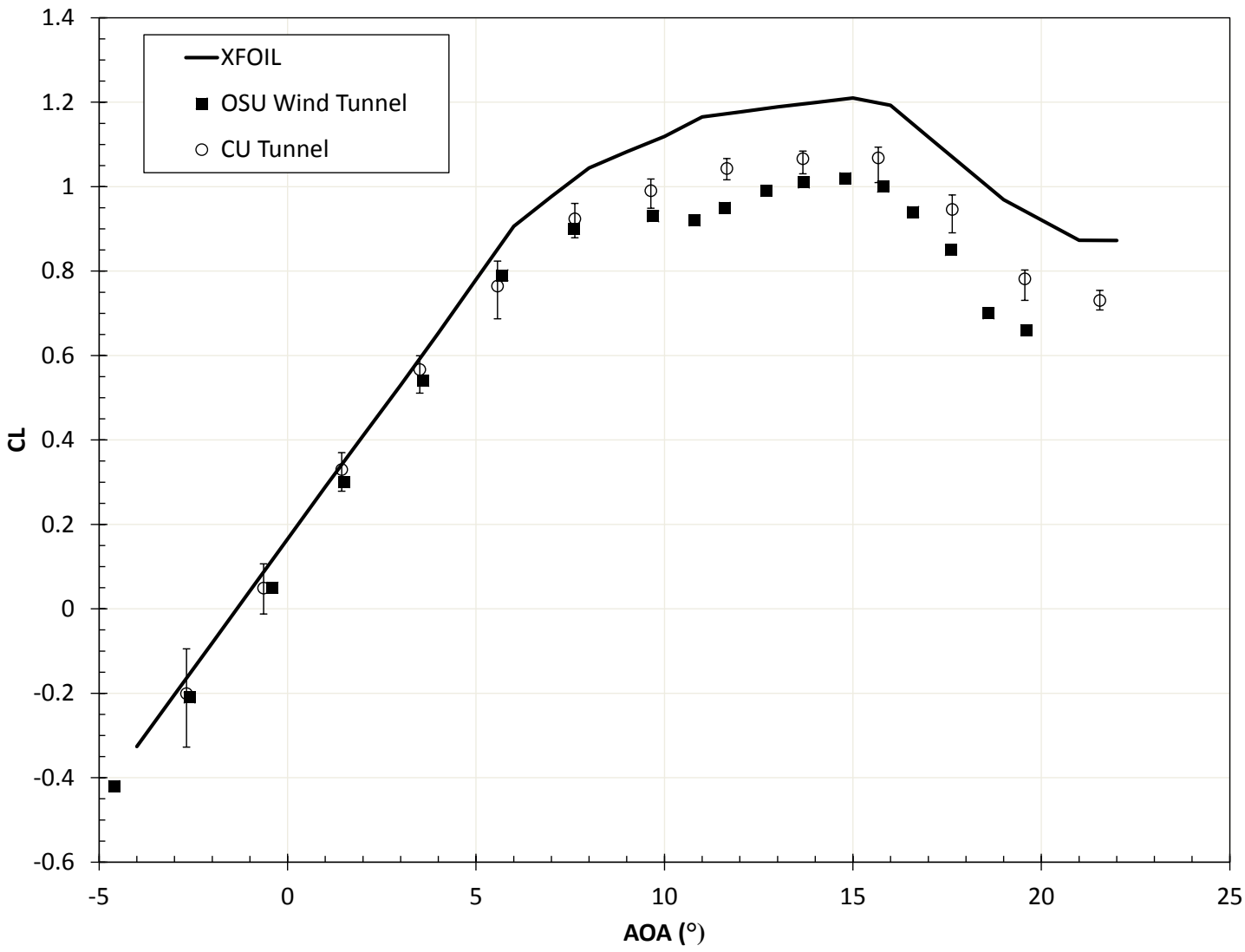

Figure 4.9: The coefficient of lift results from Carleton University (CU) compared to Ohio State University (OSU) and XFOIL at $R e \doteq 750,000$

discrepancy at higher angles of attack may be explained by the thicker trailing-edge used at CU (0.66\%) compared to the OSU model (0.27\%) [123] which would result in a minor increase in lift around the stall and post-stall region. Other differences may be attributed to disparities in e.g. turbulence intensity or aspect ratio. In the attached flow region, XFOIL exhibits a minor over-prediction of lift, however, as the flow begins to separate, the over-prediction becomes more apparent. The overprediction of lift in the stall and post-stall region is a common pitfall of XFOIL and is clearly observed here.

The drag measurements performed at CU agree very well with the OSU data in 


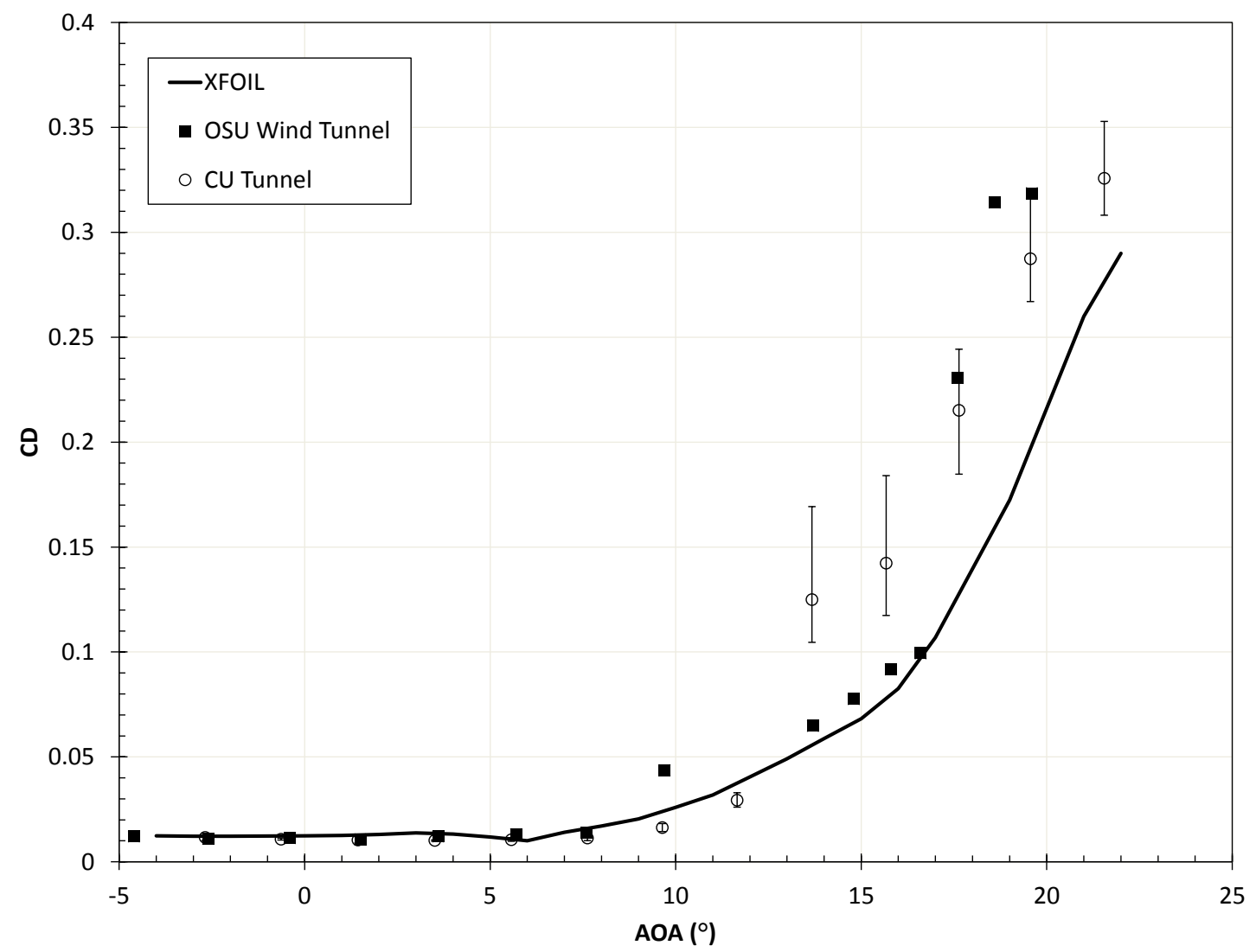

Figure 4.10: The coefficient of drag results from Carleton University (CU) compared to Ohio State University (OSU) and XFOIL at $R e \doteq 750,000$

the attached region. Interestingly, both sets of experimental data show lower values of drag than XFOIL's predictions at low angles of attack, albeit only by a small margin. Once separation begins to occur, the CU and OSU data differ, however in both cases, show higher drag values than XFOIL's predictions. These differences between experimental data sets may be due to the fact that the OSU data is solely pressure drag (the momentum drag method was found to be unreliable for unsteady, separated flow), whereas the CU data is obtained via the force sensor which is not only measuring the pressure drag, but also the skin friction drag. Furthermore, the measured force is the resultant of the forces acting across the entire airfoil span, which may exhibit some 3D flow effects near the tunnel walls which likely will cause 
fluctuating flow characteristics, and explains the rather large spread of the data at high angles of attack. Nonetheless, the similar trend in both sets of experimental data sets confirms that XFOIL struggles to accurately predict largely separated flow.

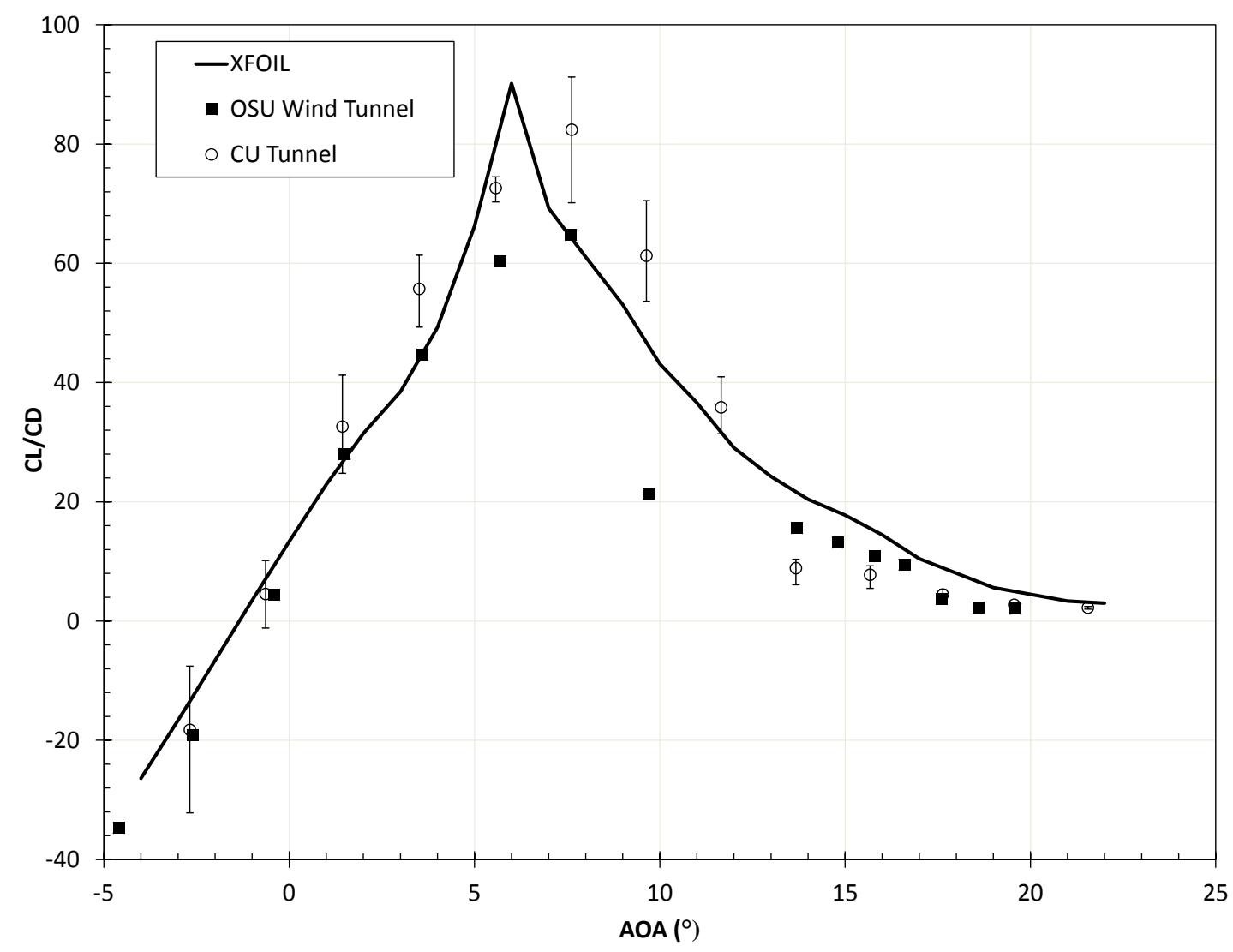

Figure 4.11: The coefficient of lift-to-drag results from Carleton University (CU) compared to Ohio State University (OSU) and XFOIL at $R e \doteq 750,000$

When comparing the lift-to-drag ratio, the slightly larger values of lift obtained at $\mathrm{CU}$, and the comparable levels of drag, result in larger lift-to-drag ratios than OSU up to about $12^{\circ}$, at which point the higher drag values act to lower the ratio. Compared to XFOIL predictions, both sets of experimental data tend to suggest that the peak $C_{L} / C_{D}$ occurs at an approximately $1^{\circ}$ higher angle of attack. The magnitude of the maximum $C_{L} / C_{D}$ obtained experimentally is hard to compare due to insufficient data 
points in the narrow angle of attack region at which it occurs, however, both cases seem to show a slight under-prediction.

The agreeable comparison of $\mathrm{CU}$ and $\mathrm{OSU}$ experimental data indicate that the experimental set-up and method developed for this work are suitable for the 2D testing of airfoils. Although there is a rather large spread in the values of post-stall drag, the trends of the data are consistent with OSU tests. Furthermore, despite the S809 being of moderate thickness and possessing a nominally thin trailing-edge, the presence and magnitude of XFOIL's lift over-prediction, and drag under-prediction found by many other researchers $[60-62,99,116]$ was observed.

\subsection{2 $\mathrm{C}_{\mathrm{T}}$ Airfoil Performance}

The $C_{T}$ airfoil designed in this work was experimentally tested at Carleton University. Of all the $30 \%$ thick airfoils designed, it possesses the largest trailing-edge thickness (4.8\%). The relatively high thickness of the airfoil, combined with the large trailingedge should result in large discrepancies between XFOIL predictions and measured values, as XFOIL is not capable of accurately predicting the performance of airfoils of this type. For this reason, the $C_{T}$ airfoil should offer further insight into differences between experimental and numerical predictions, and more importantly, should stress the importance of experimental testing for thick, flatback airfoils.

When comparing experimental $C_{L}$ values to those predicted by XFOIL, as shown in Figure 4.12, the most obvious difference lies in the stall and post-stall region where a rather large discrepancy is found. This difference comes primarily due to XFOIL's inability to predict the performance of large trailing-edge thicknesses and largely separated flows. In terms of percentage error, the XFOIL lift prediction for the $C_{T}$ airfoil exhibits a similar, albeit slightly larger difference than the S809 airfoil; 


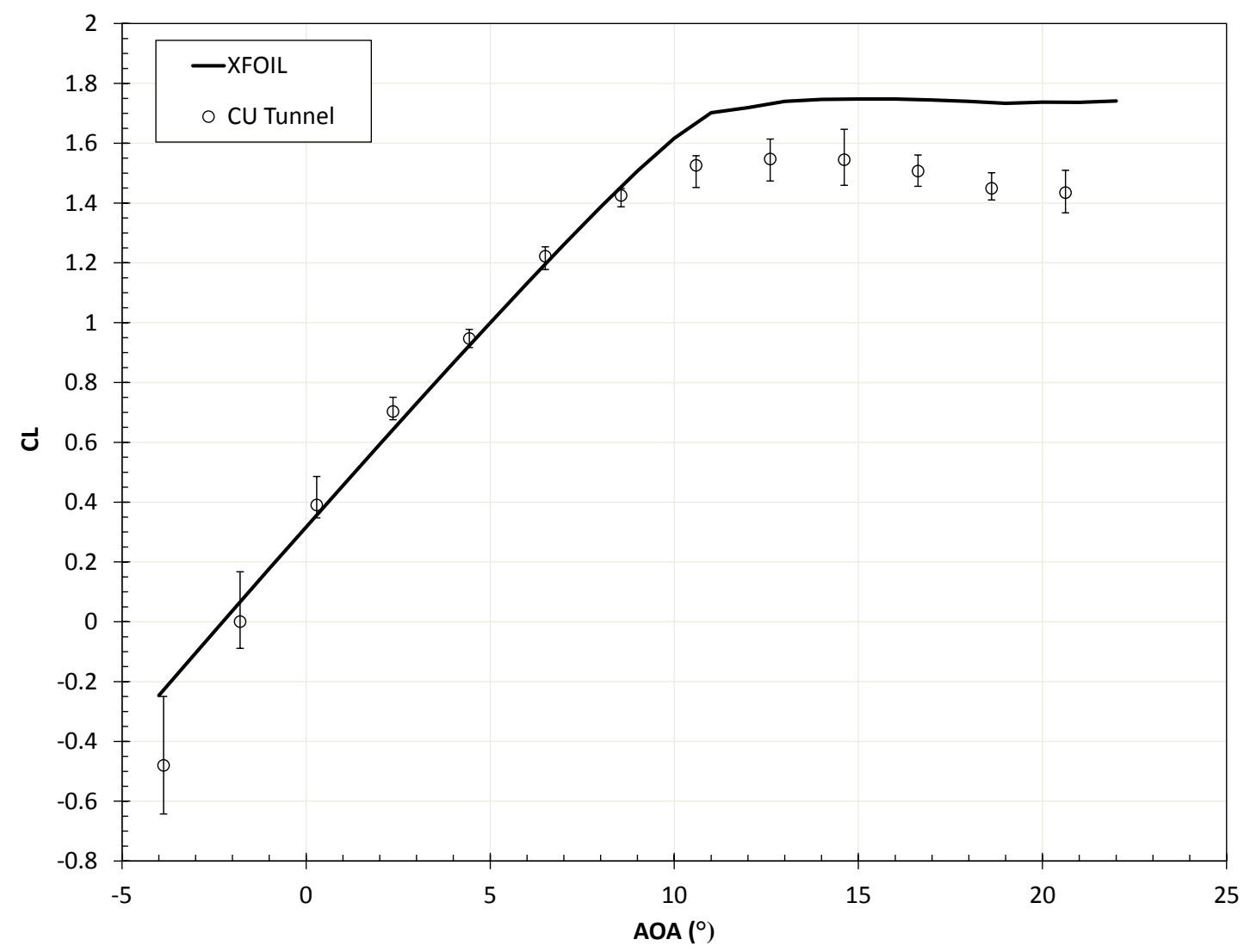

Figure 4.12: The coefficient of lift results from Carleton University (CU) compared to XFOIL at $R e \doteq 750,000$

both over estimate post-stall lift by about $13 \%$. However, on an absolute difference basis, the average over-prediction in lift for the $C_{T}$ airfoil is 0.23 units compared to 0.13 units for the S809 airfoil. It can be seen that at angles of attack below $0^{\circ}$, the experimental results diverge from the expected linear response, however the increase in error bar range show that this area is subject to larger variations in measurements. Additionally, a slight difference in the lift prediction in the linear slope region exists.

Further study is required to determine the reason for these differences, however, at low/negative angles of attack, it appears as if more negative lift force is being produced. An airfoil equipped with pressure taps along the chord line may reveal more details regarding the location of transition and separation, and offer insight 
as to why any discrepancies exist. Thus far, the results indicate that XFOIL's lift predictions become less accurate for thick, flatback airfoils and therefore must be used with caution. When designing a full rotor blade, accurate values of $C_{L}$ are essential to ensure proper aerodynamic loading, therefore a large difference in lift between predictions and reality can play an adverse role and result in poor overall wind turbine performance.

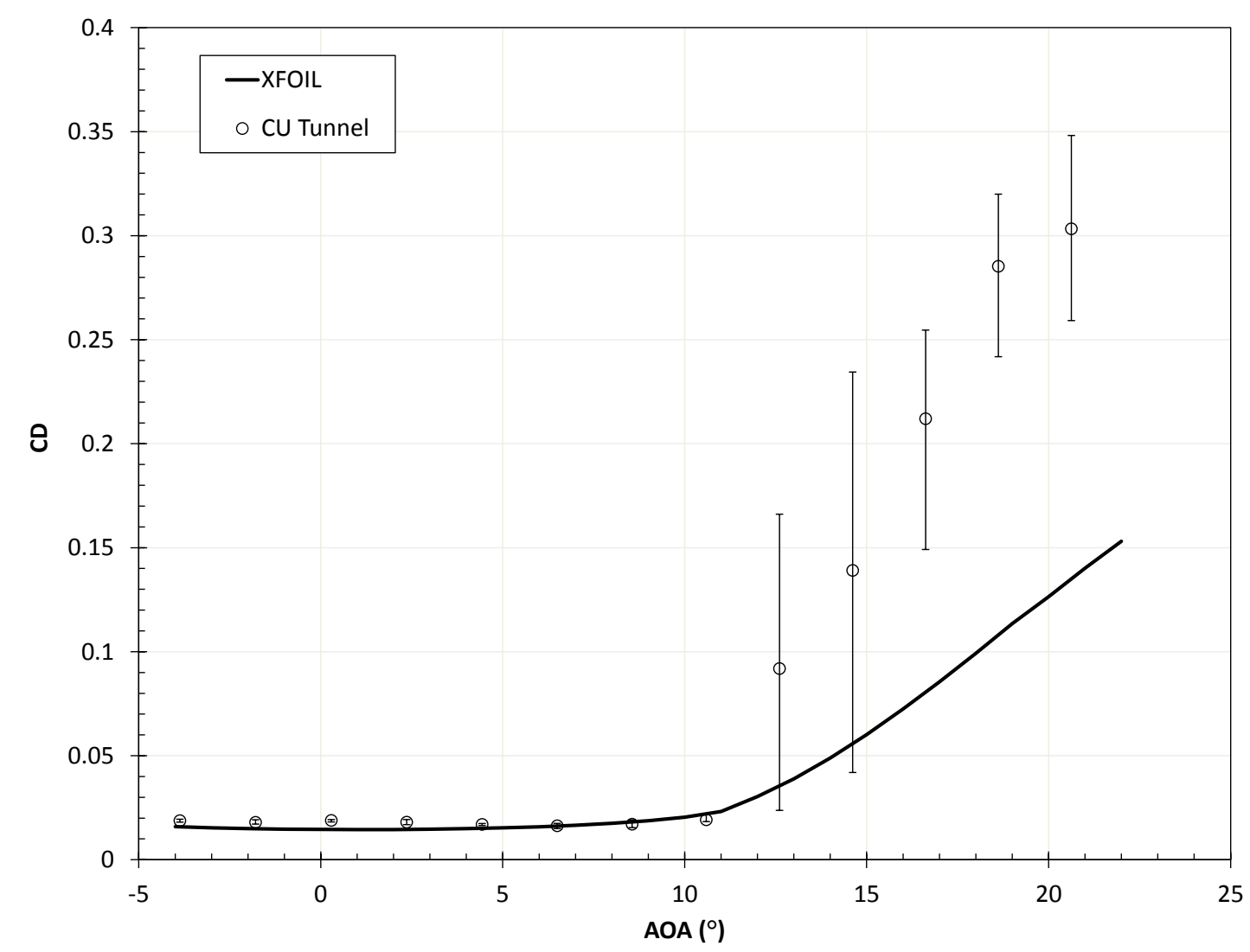

Figure 4.13: The coefficient of drag results from Carleton University (CU) compared to XFOIL at $R e \doteq 750,000$

For the (mostly-)attached flow regime, the drag is generally under-predicted by XFOIL. This tends to suggest that XFOIL does not model the increase in drag that comes as a result of a thick trailing-edge properly. As shown in Figure 4.13, once 
separation begins, the difference becomes larger, and although some values fall within the error bars, for the most part the predictions are far from reality. The large error bars at high angles of attack, to be discussed in further detail in Section 4.13, indicate that there is likely an unsteady flow which is causing variation of the separation point. Furthermore, this large spread in data was observed when using both the force sensor and momentum wake method at high angles of attack, tending to suggest it is something physical, and not simply a short-coming of the sensor itself.

The under-predictions of drag at large angles of attack by XFOIL for the $C_{T}$ airfoil are about twice that for the thinner, nominally sharp trailing-edge S809 airfoil both in terms of magnitude and percentage error (average of 0.1 units and $150 \%$ for the $C_{T}$ airfoil, respectively). This, combined with the over-prediction of lift, indicates that XFOIL is incapable of properly predicting the aerodynamic characteristics of thick, flatback airfoils, particularly in the stall and post-stall region.

In terms of lift-to-drag ratio, these differences amount to what is shown in Figure 4.14. It can be seen that the drag, which was under-predicted in the attached region, has a rather dominant effect in this area. The location of $C_{L} / C_{D \max }$ seems to be well captured, however the magnitude is slightly off. At large angles of attack, when the flow is largely separated, the measured values are consistently lower than the predictions. Once again, this is due to the over-predicted lift, and under-predicted drag. 


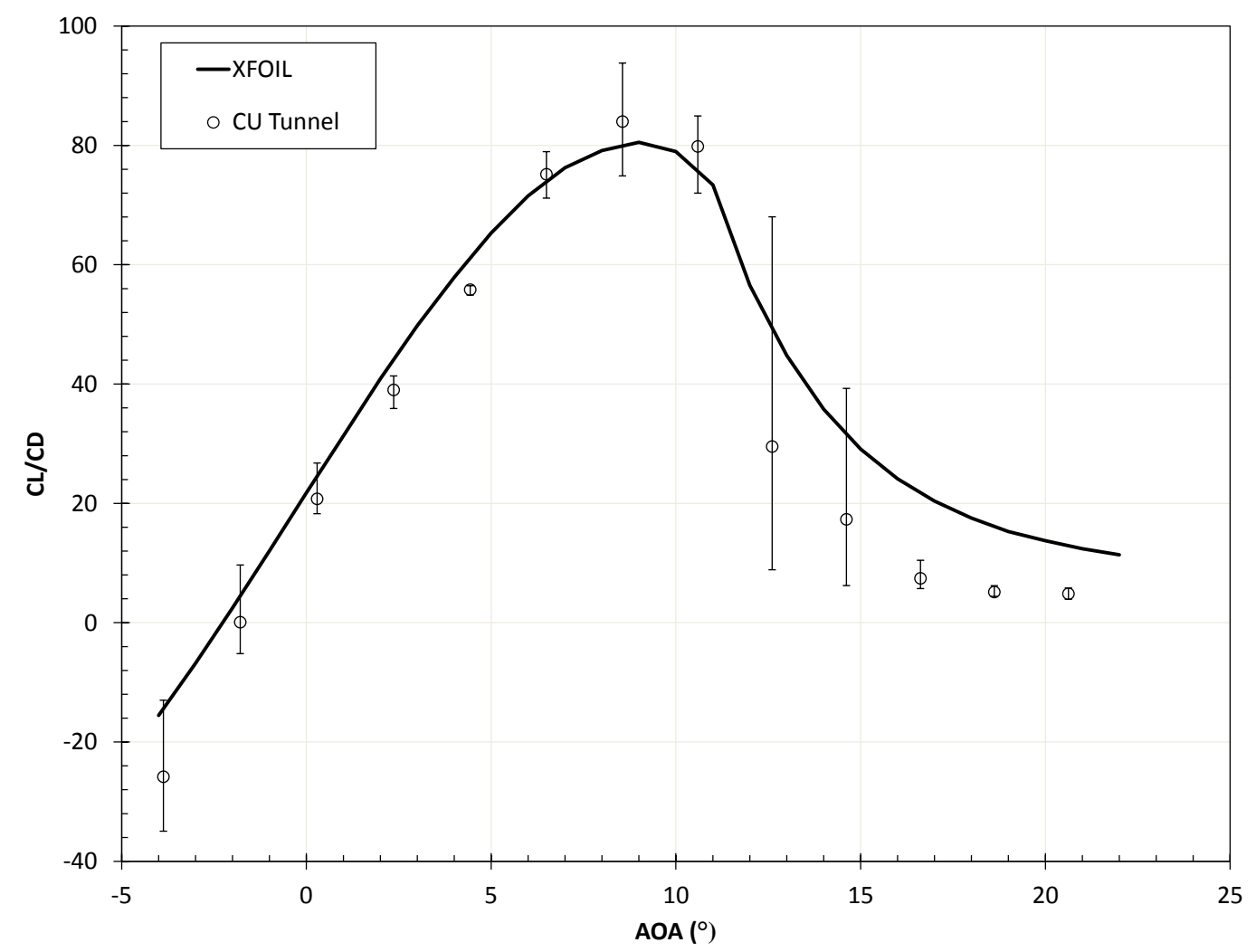

Figure 4.14: The coefficient of lift-to-drag ratio results from Carleton University (CU) compared to XFOIL at $R e \doteq 750,000$

Since the $C_{T}$ airfoil was optimized with the torque performance in mind, it is only logical to examine its measured torque direction values in comparison to predictions. Figure 4.15 shows the coefficient of torque performance of the $C_{T}$ optimized airfoil. Up until about $8^{\circ}$, the values show very good agreement, however once stall begins around $10^{\circ}$, the values diverge by quite a large margin. The large magnitudes of $C_{L}$ in relation to $C_{D}$ means that the formulation of $C_{T}$ is largely effected by $C_{L}$. Due to this, combined with the high measured drag values, the stall and post-stall $C_{T}$ values are not accurately predicted. In fact, the trend of the measured values does not agree well with predictions in the post-stall region. Furthermore, a maximum measured $C_{T}$ of about 0.26 occurs at about $10^{\circ}$ whereas the predictions indicate a maximum $C_{T}$ of about 0.51 at $22^{\circ}$, with no clear established peak yet. 


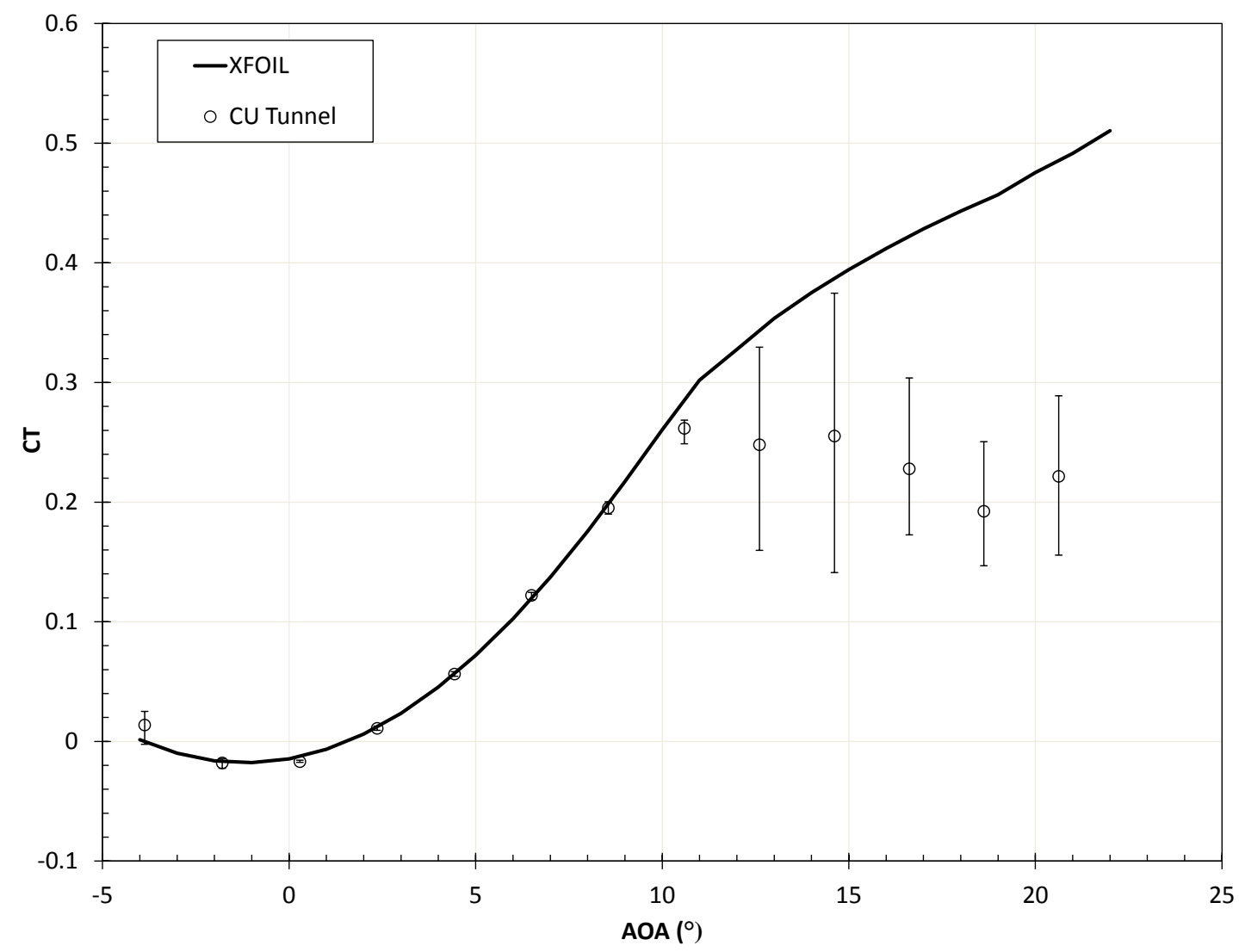

Figure 4.15: The coefficient of torque results from Carleton University (CU) compared to XFOIL at $R e \doteq 750,000$

The importance of wind tunnel testing for all airfoil designs is thus well established. The experiments presented in this work confirm that XFOIL is capable of predicting the performance of nominally sharp trailing-edge, thin airfoils with a rather good level of agreement, however, discrepancies are nonetheless still present. For thick, flatback airfoils, the deviations are larger, and demonstrate the essential need for experimental testing, as well as improved methods of predictions.

Deviations from predictions, even if minor, can have a great impact on blade design and loading. Although higher fidelity CFD techniques may provide better estimates of performance, for the optimization of airfoils, a fast computational tool such as XFOIL/RFOIL is essential. For thick, flatback airfoils, XFOIL may be modified to 
allow for better lift and drag agreement; some changes have already been implemented in RFOIL to better predict drag and maximum lift, however, room for improvement is still present. Accounting for these differences in the design phase will allow for better wind turbine airfoils to be conceived, and in turn, superior overall wind turbine performance thereby lowering the cost-of-energy of wind power.

In addition to highlighting and confirming key differences between measured and predicted values, this work also served to establish an airfoil testing set-up and method at Carleton University. The good agreement of measured values at CU for the S809 airfoil compared to the well-established OSU tests, proves that the wind tunnel setup at $\mathrm{CU}$ is capable of realizing meaningful data. Many suggested improvements to the set-up are described in Chapter 5, however a key improvement may include the implementation of pressure taps along the airfoil surface to provide pressure drag, as well as a secondary measurement technique for lift and moment. Furthermore, it may provide further details such as separation or transition point to explain why the discrepancies found exist. Additionally, an automated traverse, and pressure recording data acquisition system for the wake momentum drag method should provide more accurate values, while reducing the need for human-interaction.

\subsubsection{Uncertainty Analysis}

An uncertainty analysis provides an estimate of the true value based on the sample mean, $\bar{x}$, and its associated uncertainty values, $U_{x}$, as shown in Equation 4.13. Typically, a confidence interval based on $95 \%$ probability is used for engineering experiments, and will be used in this work.

$$
x=\bar{x} \pm U_{x}
$$


Any measurement consists of a total error which is the sum of systematic error, $S$, and random error, $R$. Systematic error is the deviation of a measured value from its true value due to the measurement system, whereas random error is the deviation in the measured value over several repetitions due to uncontrollable, random factors [134]. The overall uncertainty can be given by Equation 4.14 where $t_{\nu, 95 \%}$ is the Student $t$-Distribution at $95 \%$ confidence, and $\nu=N-1$, where $N$ is the number of repeats. In this work, $N=4$, therefore $t_{\nu, 95 \%}=3.182$ [135].

$$
U_{x}=\sqrt{S_{x}^{2}+\left(t_{\nu, 95 \%} R_{x}\right)^{2}}
$$

The systematic error for a given measurement may come from the resolution of the reading, the accuracy of the measurement device, hysteresis effects, linearity effects, bias, and more. The following sections will provide estimates for the systematic uncertainty for each measurement, followed by a total estimate for the measured value uncertainties. Although the uncertainty analysis process should be repeated for each data point, it will be shown here for the S809 and $C_{T}$ airfoil for a point in the attached region (approx 5.5 ), for which the momentum drag method is used, as well as one point in the post-stall region (approx $17.5^{\circ}$ ), for which the force sensor is used for all force measurements. The random error was estimated to be the standard deviation between repeated measurements, as shown in the equation below.

$$
R_{x}=\sqrt{\frac{\sum\left(x_{i}-\bar{x}\right)^{2}}{N-1}}
$$

All calculations make use of the following rules of addition/subtraction, multiplication/division, and exponential propagation of uncertainties where $\partial X$ represents the uncertainty in the units of measure, $X$ is the measured value, and $V$ and $\partial V$ are the resultant value and associated uncertainty, respectively [136]. To express the 
uncertainty as a percentage, simply calculate $\frac{\partial V}{V} \times 100$. Note that $Y$ and $Z$ simply represent different measured values that are involved in a given calculation.

Addition/Subtraction:

$$
\begin{gathered}
V=X-Y+Z \\
\partial V=\sqrt{\partial X^{2}+\partial Y^{2}+\partial Z^{2}}
\end{gathered}
$$

\section{Multiplication/Division:}

$$
\begin{gathered}
V=\frac{X Y}{Z} \\
\partial V=V \sqrt{\left(\frac{\partial X}{X}\right)^{2}+\left(\frac{\partial Y}{Y}\right)^{2}+\left(\frac{\partial Z}{Z}\right)^{2}}
\end{gathered}
$$

Exponents:

$$
\begin{gathered}
V=X^{n} \\
\partial V=n \frac{\partial X}{X} V
\end{gathered}
$$

When a parameter is a function of several variables, the equation listed below can be employed. It can be seen that the sensitivity, which is how the result changes with respect to a certain variable, is included in the calculation to account for differences in response to certain parameters. Note that if a parameter is assumed to be a constant, its associated uncertainty is considered to be 0 .

Functions:

$$
\begin{gathered}
V=V(X, Y, Z) \\
\partial V=\sqrt{\left(\frac{d V}{d X} \partial X\right)^{2}+\left(\frac{d V}{d Y} \partial Y\right)^{2}+\left(\frac{d V}{d Z} \partial Z\right)^{2}}
\end{gathered}
$$




\section{Density Measurements}

The measurement of the density of air is calculated according to the ideal gas law, which is dependent on atmospheric pressure, the temperature of the air, as well as the gas constant (assumed constant, $R=287 \mathrm{~J} / \mathrm{kgK}$ ). The atmospheric pressure has an uncertainty of $\pm 50 \mathrm{~Pa}$, which is half of the resolution of the data from Environment Canada, and the temperature, as measured by a type-K thermocouple, is considered to be $\pm 2.2^{\circ} \mathrm{C}$. For an average recorded atmospheric pressure of 101,025 $\mathrm{Pa}$ and tunnel temperature of $28.08^{\circ} \mathrm{C}\left(301.08^{\circ} \mathrm{K}\right)$, this can be expressed as $\pm 0.05 \%$ and $\pm 0.73 \%$, respectively, resulting in an average density of $1.169 \mathrm{~kg} / \mathrm{m}^{3} \pm 0.73 \%$ $\left(1.169 \pm 0.0085 \mathrm{~kg} / \mathrm{m}^{3}\right)$. Note that since units of Kelvin are used in the calculation of density, the temperature uncertainty is relatively small. As a worst-case scenario, the lowest recorded atmospheric pressure, and lowest recorded temperature was used to calculate an error in density of $\pm 0.75 \%$.

\section{U-tube Manometer Measurements}

The only significant measurement uncertainty in the U-tube manometer used to measure the velocity of the air, other than density of water, which can be assumed to be $1000 \mathrm{~kg} / \mathrm{m}^{3}$, arises from the resolution of the markings on the tubes. With markings every $2 \mathrm{~mm}$, it can be assumed that the greatest accuracy at which it can be read is $\pm 1 \mathrm{~mm}$, however, since the measurement is read at both ends of the U-tube, this uncertainty is $\pm 1.41 \mathrm{~mm}$. With a typical height differential in the U-tube manometer being about $93 \mathrm{~mm}$, this corresponds to $\pm 1.52 \%$.

The height difference is used to calculate the air velocity (see Equation 4.4). Applying Equations 4.16-4.23 results in an uncertainty of $\pm 0.22 \mathrm{~m} / \mathrm{s}$. With velocities typically being on the order of $40 \mathrm{~m} / \mathrm{s}$, this is $\pm 0.55 \%$. A worst-case scenario, in which the lowest typical height differential (measured in the wake of an airfoil), combined 
with the highest density uncertainty results in $\pm 2.18 \%( \pm 0.32 \mathrm{~m} / \mathrm{s})$.

\section{Geometric Measurements}

Since the forces acting on the airfoil are dependent on the planform area of the airfoil, an accurate geometric measure must be established. The machine used to CNC the airfoils has an accuracy of about $\pm 0.002 "$, therefore can be considered dimensionally accurate. Although the foam that was added to either end of the airfoil to prevent leakage was measured using calipers accurate to $\pm 0.005 \mathrm{~mm}$, an accuracy for the measurement of the foam of $\pm 0.05 \mathrm{~mm}$ was used to account for any compression of the foam, or unevenness of the surface. For the span of the airfoil the uncertainty is the combination of the CNC machine accuracy, plus the measurement of the foam on either side (hence measured twice) giving $\pm 0.087 \mathrm{~mm}$. For a span of $0.508 \mathrm{~m}$ this equates to $\pm 0.017 \%$. For the chord length of $0.3 \mathrm{~m}$, only the uncertainty in the CNC machine is taken into account resulting in $\pm 0.017 \%$ as well. Therefore, the total area can be considered to be $\pm 0.024 \%$.

The 1/4" markings on the pitot-static tube were made using a ruler with indications every 1/16" (1.6 mm), therefore the uncertainty can be considered to be $\pm 1 / 32$ " $( \pm 0.8 \mathrm{~mm})$. It is estimated that the tube can be positioned by the user with an accuracy of $\pm 1.0 \mathrm{~mm}$, therefore, when combined with the uncertainty associated with the markings themselves, a total uncertainty of $\pm 1.3 \mathrm{~mm}$ results.

The angle measurements are subject to uncertainty due to the device used to create the angle indications on the bristol board, as well as uncertainty due to the resolution of the markings. Combined, the $\pm 0.3^{\circ}$ device and $\pm 0.5^{\circ}$ resolution uncertainties result in a total uncertainty of $\pm 0.58^{\circ}$. 


\section{Lift and Drag Forces}

The ATI Delta 6-component force sensor has an accuracy of $\pm 1.5 \%$ (95\% confidence) of full-scale which works out to $\pm 9.9 \mathrm{~N}$. As mentioned earlier, the force sensor was used for all lift and moment force measurements, as well as post-stall drag (angles of attack greater than $10^{\circ}$ ) whereas for pre-stall drag, the wake momentum method was employed using a pitot-static tube. It should be noted that although the Omega OM-DAQ-USB2401 data acquisition device used to record the data has an associated error, it is considered to be insignificant relative to the other measurements and therefore not included in the uncertainty analysis.

The forces recorded by the force sensor were divided by the dynamic pressure multiplied by the airfoil area to obtain the non-dimensional coefficients. The drag force multiplied by 2 , as calculated by the wake momentum method, was divided by the velocity squared multiplied by the chord (see Equation 4.3) since it was only measured in a plane. The uncertainty in the wake method must account for the fact that the integration in Equation 4.3 is performed by summing the velocity and geometric measurements therefore the associated uncertainty is the combination of the 10 (typically) measurements within the wake. Table 4.1 summarizes the uncertainties associated with the $\mathrm{S} 809$ and $C_{T}$ airfoil for angles of attack in the pre-stall and poststall region. 
Table 4.1: Sample measured values and their associated uncertainties (95\% confidence) for the S809 and $C_{T}$ airfoils

\begin{tabular}{|c||c|c|c|}
\hline \multicolumn{4}{|c|}{ Sample Uncertainties } \\
\hline Airfoil & Angle of Attack & $C_{L}$ & $C_{D}$ \\
\hline S809 & $5.57 \pm 0.58^{\circ}$ & $0.7646 \pm 0.1983$ & $0.0105 \pm 0.0054$ \\
S809 & $17.64 \pm 0.59^{\circ}$ & $1.0682 \pm 0.1416$ & $0.1423 \pm 0.1046$ \\
\hline$C_{T}$ & $6.50 \pm 0.58^{\circ}$ & $1.2220 \pm 0.1250$ & $0.0163 \pm 0.0064$ \\
$C_{T}$ & $16.62 \pm 0.58^{\circ}$ & $1.5070 \pm 0.1541$ & $0.2120 \pm 0.1581$ \\
\hline
\end{tabular}

As can be seen from the table, rather high levels of uncertainty exist, however, the purpose of this work was not to acquire exact data, but to establish a means of testing airfoils, and to highlight the importance of wind tunnel testing as part of the design process. Ignoring the contribution of the t-Student multiplier, the systematic uncertainty was on average about $2 \times$ higher than the random error. This high level of uncertainty is especially detrimental to the experimental drag force data where the magnitudes of forces are low. In all cases, the majority of the systematic uncertainty stems from the force sensor measurements which is better suited for larger magnitude forces. Nonetheless, the trend of the measured data agrees well, and deviates from predictions as expected. Furthermore, with the basis of the testing set-up already developed, several improvements can be implemented in the future.

Besides the force sensor contributing to a large portion of the uncertainty due to it not being perfectly suited for the measurements at hand, the total uncertainty also incorporates random error. At high angles of attack, the $C_{T}$ airfoil exhibited standard deviations of drag measurements approximately $1.8 \times$ higher than that of the thin trailing-edge S809 airfoil. This large spread in data was found no matter whether the force sensor, or momentum wake method was used, and is likely due to 3D flow patterns over the airfoil caused by tunnel-airfoil junction effects, largely 
separated flow, and the blunt trailing-edge of the $C_{T}$ airfoil which causes unsteady flow fluctuations. As vortices are shed from the thick trailing-edge, the point of transition, separation and effective angle of attack all vary which in turn alter the forces acting on the airfoil. This was observed while taking U-tube manometer readings where the levels of water would fluctuate to a much higher degree than during the testing of the S809. The presence of 3D flow patterns were found in other flatback airfoil experiments where flow visualization techniques revealed the formation of stall cells [137] which are large, localized regions of separated flow found on the suction side of airfoils at high angles of attack which may exhibit random formation or destruction [138]. In another study, a spanwise variation in drag was observed, despite efforts to minimize 3D effects [139]. This spanwise variation resulted in drag values ranging from $C_{D}=0.10$ to $C_{D}=0.26$, thereby highlighting the significant impact 3D flow may have. Flow visualization techniques such as Particle Velocimetry Imaging (PIV), oil flow, or flow tufts may help to reveal the presence of 3D flow.

Upon further analysis at high angles of attack, it was found that for both the S809 and $C_{T}$ airfoil, whenever high lift was measured, low drag was found and viceversa. This suggests that in the high lift-low drag case, the flow is more attached than in the low lift-high drag case, and indicates that the degree of flow separation is inconsistent between trials. Furthermore the variation of the forces acting on the airfoils, particularly the $C_{T}$ airfoil, were noticeable in the form of slight vibrations of the airfoil in the test section at high angles of attack. This aeroelastic effect would also cause variance in the effective angle of attack, and in turn forces acting on the airfoil, which potentially may have contributed to the relatively large spread of the measured data. 
Although 4 repeats of all tests were performed in this work, the sensitive and unsteady nature of the flow over the airfoils resulted in a large spread in data. Performing more repeats should give a more representative data set and will aid in reducing the influence of random error. Investigation into, and reduction of any unsteady, 3D effects would further aid in minimizing the spread of measured data. Equipment better suited for the measurements at hand, e.g. a more accurate force sensor, separate force sensors for lift and drag, or pressure taps should aid in reducing the systematic error. Additionally, faster and longer data acquisition rates will allow for filtering of unsteady flow fluctuations and noise (e.g. wind tunnel vibrations caused by the fan and motor) when post-processed in the frequency domain.

Despite the fact that the intention of this work was not to develop an airfoil with superior performance than the $\mathrm{S} 809$, from these results it can be seen that the $C_{T}$ airfoil offers comparable $C_{L} / C_{D}$ values to the $\mathrm{S} 809$, albeit at much higher levels of $C_{L}$. Not only does this allow for a more slender design of rotor blade, but the thicker $C_{T}$ airfoil exhibits much higher levels of $I_{X X}$ due to its large trailing-edge and higher overall thickness. Furthermore, the $C_{L} / C_{D}$ performance of the $C_{T}$ airfoil exhibits less of a pronounced peak meaning that it is less sensitive to non-ideal operating conditions. 


\section{Chapter 5}

\section{Conclusions and Future Work}

The following sections highlight the findings of this thesis and propose future work to be performed; the sections are categorically divided into numerical and experimental work.

\section{$5.1 \quad$ Numerical Work}

The goal of the numerical work involved in this thesis was to obtain a better understanding of the wind turbine airfoil design process. General consensuses of desirable aerodynamic characteristics have been established in the literature, but the means of establishing them, and to what extent, are not clear- particularly in a quantitative sense.

\subsubsection{Conclusions}

In order to examine how design constraints and objectives affect the airfoil shape and performance, a means of designing optimal airfoils was established. A program was written in MATLAB to optimize the aerodynamic and structural performance of airfoils. A Bézier function with 13 control points was used to generate unique 
airfoil shapes, which were aerodynamically evaluated using XFOIL and structurally evaluated using their sectional moment of inertia. Using MATLAB's multi-objective genetic algorithm, gamultiobj, the 200 airfoil shapes that make up a single population, were used to evolve the airfoils over a maximum of 100 generations to achieve the specified design constraints and objectives. The result was either a single, optimal airfoil (if only optimized for aerodynamics) or a Pareto front of multiple airfoil designs with varying degrees of aero-structural performance.

Using the aforementioned optimization tool, the effect of the aerodynamic evaluator $\left(C_{T}, C_{L} / C_{D}, C_{T} / C_{N}\right)$ on the airfoil design was determined. Under a specified set of constraints, the $C_{T}$ airfoil naturally tended to favor roughness insensitivity, as well as high lift, at the expense of high drag, whereas the $C_{L} / C_{D}$ airfoil sacrifices roughness insensitivity and high maximum lift, to ensure low drag and therefore high $C_{L} / C_{D}$. Finally, the $C_{T} / C_{N}$ exhibits properties in between those of the $C_{L} / C_{D}$ and $C_{T}$ airfoils. The $C_{T}$ airfoil shows attractive properties for the inner region of the blade where high lift (and in turn, torque) and sectional moment of inertia are desired. In all cases, the airfoil optimized for its respective aerodynamic evaluator showed the best performance in that category proving that the optimizer is effective.

Through the present author's experience designing airfoils, as well as through discussion and feedback from leading experts, the importance of proper selection of design constraints and objectives became apparent. For example, it is indeed possible to design an airfoil using $C_{L} / C_{D}$ as the aerodynamic evaluator that is both roughness insensitive, as well as exhibits high lift characteristics, however, it must be imposed in the design phase. Similarly, a $C_{T}$ optimized airfoil may be designed to also possess a good $C_{L} / C_{D}$, however, constraints that specifically aid in this area must be included. A Taguchi method was used to examine how specific design constraints and objectives impact the airfoil outcome. It was found that both weight and $C_{L}$ Max will have the 
largest influence on the airfoil performance, and that with an increase in $C_{L}$ ratio (i.e. a more strict roughness insensitivity criteria), generally a decrease in $C_{L} / C_{D}$ performance results.

The choice of design constraints and objectives is surely driven by the desirable outcome for a particular design, however, knowing the sensitivity of the design outcome to the constraints is vital to make informed design decisions. A family of 6 airfoils, ranging in thickness from 21-36\%, was designed. All airfoils possess a high maximum lift coefficient of at least 1.9, and depending on the thickness, the ratio of $C_{L}$ between forced and free conditions ranged from $75-97 \%$. The goal of this design was to maximize $C_{L} / C_{D}$ over a $6^{\circ}$ angle of attack range, with the upper limit located approximately $4^{\circ}$ away from $\alpha_{C L M a x}$. The sectional moment of inertia, $I_{X X}$, was maximized as a design objective for each design, and in order to ensure structural compatibility, the maximum thickness was constrained to occur between $27-32 \%$ of the chord length. All airfoils exhibited similar performance under clean conditions when compared to other airfoils, and showed superior performance under rough conditions.

\subsubsection{Future Work}

The solid foundation of airfoil design knowledge, along with the optimization tool developed throughout this thesis allows for many opportunities for future work. Amongst those, the most promising and feasible include:

- Including further constraints to ensure good geometric compatibility such as curvature, and location of maximum camber.

- The inclusion of a higher fidelity aerodynamic solver such as RFOIL or CFD. RFOIL would still allow for quick computations, however, would provide better 
predictions around stall and allow for the inclusion of rotational effects into the design. CFD would offer accurate solutions of the airfoil flow, however, requires more computing power.

- The use of a more accurate structural evaluation of the airfoil. A tool such as BECAS [140] would allow for finite-element based evaluation of an airfoil cross section's stiffness, stresses, and failure based on shape, material selection, and layout. The inclusion on the sectional moment of inertia perpendicular to the chord, $I_{Y Y}$ (stiffness in the edgewise direction), in the structural evaluation may also be included; as blades grow in length, their mass and in turn gravitational loads, make edgewise stiffness a growing concern. Additionally, the torsional stiffness, $J$, of the airfoil cross-section may be included as well.

- The implementation of an aero-acoustic model such that the design takes into account aerodynamics, structure, as well as noise. This will likely result in thinner trailing-edges which will help reduce noise emission, particularly for airfoils suited for the outboard region of the blade.

- The use of a hybrid optimization which uses a genetic algorithm to search a large domain space, followed by a gradient based optimization technique to refine the design and find the absolute optimal. This hybrid technique will ensure that the best possible design is obtained within a large domain space, without the risk of getting stuck at a local optima. This technique may make use of XFOIL/RFOIL within the genetic algorithm, followed by CFD for the gradient based optimization.

- The simultaneous optimization of airfoil design within a full rotor blade optimization. This technique will allow for the design of an airfoil (and rotor blade) which takes into account the local conditions and characteristics at a particular 
point on the blade, and at neighboring sections. Better structural and aerodynamic compatibility will likely result and will ensure that the resulting design considers the global (overall) design objectives and constraints and not just the local constraints and objectives unique to each airfoil cross-section.

\subsection{Experimental Work}

Although today's methods of predicting airfoil performance such as XFOIL, or CFD are quite accurate, they cannot fully capture the airfoil characteristics to a degree which is truly representative of reality. For this reason, any new airfoil design must be verified in a wind tunnel to ensure that it performs as expected, and if it doesn't, the information gathered can be used to improve current prediction methods. The goal of the experimental work in this thesis was to establish a means of testing airfoils at Carleton University, as well as experimentally characterize select airfoils to emphasize the need for testing as part of the design process, particularly for thick flatback airfoils.

\subsubsection{Conclusions}

Using the low speed wind tunnel in room ME3224 at Carleton University, an airfoil testing set-up was established. Airfoils manufactured from Renshape spanned the height of the tunnel with foam at each end ensuring that leakage at the tips was minimized. A stainless steel rod integrated into the airfoil extends outside of the test section and mounts to an ATI-Delta 6-component force sensor. The mount allows for the angle of attack to be altered and transfers the airfoil loads to the force sensor which are then used to derive lift, drag and moment coefficients. A pitot-static tube located 1 chord length downstream of the airfoil was used to calculate drag in the attached region using the wake momentum drag method. 
The newly developed set-up was validated for an existing wind turbine airfoil, the S809, against other experimental data, as well as XFOIL. From the validation, agreeable results from Carleton University were established when compared to Ohio State University wind tunnel results. Discrepancies, although minor, may be attributed to differences in trailing-edge thickness, wind tunnel flow characteristics, and measurement techniques. In both cases, XFOIL over-predicts lift and under-predicts drag in the stall and post-stall region despite the S809 being a moderate thickness (21\%) with a nominally thin trailing-edge.

With the wind tunnel set-up producing agreeable results, a select airfoil designed by the current author was tested. The $C_{T}$ airfoil was chosen since it should provide the most noticeable differences between XFOIL's predictions and experimental data due to its relatively high $30 \%$ thickness and large $4.8 \%$ thick trailing-edge. As expected, XFOIL was not capable of predicting the lift and drag performance in the stall and post-stall region with a high degree of accuracy. On a percentage basis, the errors of the lift predictions were only slightly larger for the $C_{T}$ airfoil when compared to the S809 airfoil, however, on an absolute basis were about $2 \times$ greater. The errors in drag predictions were approximately twice that of the S809 on both an absolute difference basis, and percentage error basis. These differences in lift and drag performance can be greatly detrimental to the rotor blade design if they are not known or accounted for ahead of time.

The wind tunnel tests of both the S809 and $C_{T}$ airfoil reveal the short-comings of XFOIL and affirm the need for experimental airfoil characterization. Thicker airfoils, with flatback profiles are becoming more prominent in blade designs and as seen from this work, predictions from XFOIL cannot fully capture the flow characteristics for airfoils of this type. The airfoil design, which is in this case reliant on XFOIL, will inherently be influenced by the accuracy of XFOIL. Further testing of thick flatback 
airfoils may be used to modify and improve XFOIL's prediction capabilities such that more representative airfoil designs may be obtained.

\subsubsection{Future Work}

The 2D airfoil wind tunnel test set-up developed in this work has successfully highlighted XFOIL's inability to accurately predict the lift and drag characteristics of thick, flatback airfoils. However, the large degree of uncertainty that comes primarily due to the force sensor, as well as the unsteady nature of the flow, leaves several areas to be improved. The following points will discuss various means of improving the testing process, as well as further work to be performed in the future.

- The inclusion of pressure taps along the airfoil surface. This would not only provide a secondary, and potentially more accurate, means of measuring lift, drag, and moment, but also provide further information such as transition, separation, and pressure magnitudes over the airfoil surface.

- The design of a traverse system with a digital pressure sensor to be used to measure momentum drag. This will allow for the automated, and more accurate, recording of velocities in the wake of the airfoil. Alternatively, a wake-rake, consisting of several dozen pitot tubes at fixed intervals (resembling the shape of a comb) could be used.

- The use of flow visualization techniques such as PIV, flow tufts, or oil-flow, to identify 3D flow structures. This will be useful in further understanding their effects and subsequent minimization.

- The design of a system which transfers the lift and drag forces to separate sensors, each of which is better suited for the measurements at hand. Alternatively, 
a custom 6-component force sensor may be purchased such that the set-up can remain relatively unchanged.

- The implementation of an accurate, digital angle of attack measurement technique which would facilitate automated testing and reduce the potential for human error. Potential sensors include a rotary encoder or rotary potentiometer.

- A more structurally rigid system such that any aeroelastic effects are minimized.

- Simulating leading-edge roughness using zig-zag tape (trip strip) or even a roughness profile derived from in-field contaminated turbine blades. The airfoil's performance under forced transition conditions can be compared to XFOIL predictions.

- The collection of several airfoil's data to use in the modification of the XFOIL source code to account for larger thicknesses and flatback profiles. This empirical data would help in the improvement of XFOIL's prediction capabilities for thick profiles.

\subsection{Concluding Remarks}

This thesis was aimed at establishing the (near) start-to-finish design process of wind turbine airfoils, with a specific focus on how different design parameters affect the outcome. Firstly, a literature review provided the background, as well as the current state-of-the-art, of the airfoil design process. Next, an airfoil optimization tool was developed by coupling MATLAB's gamultiobj with XFOIL, such that in-house airfoil designs could be created to help examine the finer details of the design process. This tool allowed for the variation of several design parameters to study their influence 
on the resultant airfoil shape and performance. With the obtained design knowledge and experience, a wind turbine airfoil family was developed with constraints and objectives reflective of today's wind turbine trends and characteristics. Finally, an experimental set-up was developed, validated and used to test $2 \mathrm{D}$ airfoil sections. The results of this highlight the importance of experimental testing as part of the design process, as oftentimes numerical prediction techniques fail to properly simulate the stall and post-stall regions. The data collected can be used to improve numerical prediction techniques (not performed in this work) such that future designs perform closer to expectations. 


\section{List of References}

[1] E. Hau, Wind Turbines: Fundamentals, Technologies, Applications, Economics. Springer, 2 ed., 2005.

[2] P. Brondsted and R. Nijssen, eds., Advances in Wind Turbine Blade Design and Materials. Woodhead Publishing, 2013.

[3] Thresher, R. and Robinson, M. and Musial, W. and Veers, P., Wind Turbine Technology, ch. 3: Evolution of Modern Wind Turbines Part B: 1988 to 2008, pp. 171-202. ASME Press, 2009.

[4] M. Ge, D. Tian, and Y. Deng, "Reynolds Number Effect on the Optimization of a Wind Turbine Blade for Maximum Aerodynamic Efficiency," in Journal of Energy Engineering, 2014.

[5] O. Ceyhan, "Towards 20MW Wind Turbine: High Reynolds Number Effects on Rotor Design," in 50th AIAA ASM Conference, (Nashville, TN), Jan. 2012.

[6] "V164-8.0 MW Breaks World Record for Wind Energy Production," Press Release, MHI Vestas, Aarhus, DK, Nov. 2014.

[7] S. Schreck, L. Fingersh, K. Siegel, M. Singh, and P. Medina, "Rotational Augmentation on a 2.3-MW Rotor Blade with Thick Flatback Airfoil Cross Sections," in 51st AIAA Aerospace Sciences Meeting including the New Horizons Forum and Aerospace Exposition, (Dallas, TX), Jan. 2013.

[8] Enercon, "Windblatt- A new family of ENERCON rotor blade profiles." Magazine, Apr. 2014.

[9] K. Fukami, "Patent EP 2604856 A1- Wind turbine blade, wind power generation device provided with same, and design method for wind turbine blade," June 2013. 
[10] K. Standish and S. Herr, "Patent US 7883324 B2- Wind turbine airfoil family," Feb. 2011.

[11] H. Xu, W. Shen, W. Zhu, and C. Liu, "Aerodynamic Analysis of Trailing Edge Enlarged Wind Turbine Airfoil," in The Science of Making Torque from Wind, Journal of Physics, 2014.

[12] TPI Composites, "Innovative Design Approaches for Large Wind Turbine Blades Final Report," tech. rep., Sandia National Laboratories, May 2004.

[13] L. Viterna and D. Ancona, Wind Turbine Technology, ch. 4: Commercial Wind Turbine Systems and Applications, pp. 203-280. ASME Press, 2009.

[14] R. Wilson, Wind Turbine Technology, ch. 5: Wind Turbine Aerodynamics Part A: Basic Principles, pp. 281-350. ASME Press, 2009.

[15] R. Wiser and M. Bolinger, 2014 Wind Technologies Market Report. Oak Ridge, TN: U.S. Department of Energy, 2014.

[16] Mone, C. and Smith, A. and Maples, B. and Hand, M., "2013 Cost of Wind Energy Review," tech. rep., National Renewable Energy Laboratory, Golden, CO, Feb. 2015.

[17] M. Vila and M. Alfaro, "New Airfoil Family Design for Large Wind Turbine Blades," in AIAA SciTech 33rd Wind Energy Symposium, (Kissimmee, FL), Jan. 2015.

[18] J. Cotrell, T. Stehly, J. Johnson, J. Roberts, Z. Parker, G. Scott, and D. Heimiller, "Analysis of Transportation and Logistics Challenges Affecting the Deployment of Larger Wind Turbines: Summary of Results," tech. rep., National Renewable Energy Laboratory, Golden, CO, Jan. 2014.

[19] Enercon, "Windblatt- Premiere for new blade concept." Magazine, Jan. 2014.

[20] B. Jayaswal and C. Patton, The Design for Trustworthy Software Compilation Taguchi Methods and Optimization for Robust Software. Prentice Hall, Mar. 2007.

[21] F. White, Fluid Mechanics, ch. 3.7: Frictionless Flow: The Bernoulli Equation. McGraw-Hill, 5 ed., 2003. 
[22] J. Anderson, Fundamentals of Aerodynamics. New York, NY: McGraw-Hill, 5 ed., 2010.

[23] M. Hansen, Aerodynamics of Wind Turbines. London, UK: Earthscan, 2 ed., 2008.

[24] W. Shen, W. Zhu, and H. Madsen, "New airfoils for high rotational speed wind turbines: Design and Analysis of Tip Section Airfoils Taking into Account Compressible Effects," tech. rep., Innwind.EU, Sept. 2015.

[25] M. Drela, "XFOIL: An analysis and design system for low reynolds number airfoils," in Low Reynolds Number Aerodynamics (T. J. Mueller, ed.), vol. 54, (Notre Dame, IN), pp. 1-12, 1989.

[26] S. Law and G. Gregorek, "Wind Tunnel Evaluation of a Truncated NACA 64621 Airfoil for Wind Turbine Applications," tech. rep., National Aeronautics and Space Administration, Cleveland, OH, July 1987.

[27] K. Bullivant, "Tests of the NACA 0025 and 0035 Airfoils in the Full-Scale Wind Tunnel," Tech. Rep. 708, National Advisory Committee for Aeronautics, Washington, D.C., 1941.

[28] Abbott, I. and von Doenhoff, A. and Stivers, L., "Summary of Airfoil Data," Tech. Rep. 824, National Advisory Committee for Aeronautics, Langley Field, VA, 1945.

[29] K. Standish and C. Van Dam, "Analysis of Blunt Trailing Edge Airfoils," in 41st Aerospace Sciences Meeting and Exhibit, (Reno, NV), Jan. 2003.

[30] T. Sant, G. van Kuik, and G. van Bussel, "Estimating the Angle of Attack from Blade Pressure easurements on the NREL Phase VI Rotor Using a Free Wake Vortex Model: Axial Conditions," Wind Energy, pp. 549-577, Sept. 2006.

[31] F. Grasso, "Hybrid Optimization for Wind Turbine Thick Airfoils," in 53rd AIAA/ASME/ASCE/AHS/ASC Structures, Structural Dynamics and Materials Conference, (Honolulu, HI), American Institute of Aeronautics and Astronautics, Apr. 2012.

[32] S. Hoerner, "Base drag and thick trailing edges," Journal of Aeronautical Sciences, vol. 17 10, pp. 622-628, 1950. 
[33] S. Hoerner, Fluid-Dynamic Drag, ch. 3, p. 22. Bakersfield, CA: Hoerner Fluid Dynamics, 1965.

[34] H. Smith and R. Schaefer, "Aerodynamic Characteristics at Reynolds Numbers of $3.0 \times 10^{6}$ and $6.0 \times 10^{6}$ of Three Airfoil Sections Formed by Cutting Off Various Amounts from the Rear Portion of the NACA 0012 Airfoil Section," Tech. Rep. 2074, National Advisory Committee for Aeronautics, Langley, VA, Apr. 1950.

[35] V. Ramjee, E. Tulapurkara, and V. Balabaskaran, "Experimental and Theoretical Study of Wings with Blunt Trailing Edges," Journal of Aircraft, vol. 23, Apr. 1986.

[36] J. Sato and Y. Sunada, "Experimental Research on Blunt Trailing-Edge Airfoil Sections at Low Reynolds Numbers," AIAA Journal, vol. 33, Nov. 1995.

[37] M. Post, R. Jones, A. Denton, and R. Millard, "Characterization of a Flatback Airfoil for use in Wind Power Generation," in 46th AIAA Aerospace Sciences Meeting and Exhibit, (Reno, NV), Jan. 2008.

[38] J. Baker, E. Mayda, and C. van Dam, "Experimental and Computational Analysis of Thick Flatback Wind Turbine Airfoils," in 44th AIAA Aerospace Sciences Meeting and Exhibit, (Reno, NV), Jan. 2006.

[39] J. Baker, C. van Dam, B. Gilbert, and D. Berg, "Flatback Airfoil Wind Tunnel Experiment," tech. rep., Sandia National Laboratories, Apr. 2008.

[40] J. Baker, Drag Reduction of a Blunt Trailing-Edge Airfoil. PhD thesis, University of California Davis, 2009.

[41] C. Metzinger, J. Baker, J. Grobbel, and C. van Dam, "Effects of Splitter Plate Length on Aerodynamic Performance \& Vortex Shedding on Flatback Airfoils," in 50th AIAA Aerospace Sciences Meeting including the New Horizons Forum and Aerospace Exposition, (Nashville, TN), Jan. 2012.

[42] S. Yang and J. Baeder, "Aerodynamic Drag and Aeroacoustic Noise Mitigation of Flatback Airfoil with Spanwise Wavy Trailing Edge," in AIAA SciTech 33rd Wind Energy Symposium, (Kissimmee, FL), Jan. 2015.

[43] R. Seele, C. Chen, C. Bhamburkar, and I. Wygnanski, "Some Effects of Blowing, Suction and Trailing Edge Bluntness on Flow Separation from Thick Airfoils; 
Computations \& Measurements," in 29th AIAA Applied Aerodynamics Conference, (Honolulu, HI), June 2011.

[44] M. El-Gammal, J. Naughton, and H. Hangan, "Drag Force Balance of a Blunt and Divergent Trailing-Edge Airfoil," Journal of Aircraft, vol. 47, Jan. 2010.

[45] D. Berg and J. Zayas, "Aerodynamic and Aeroacoustic Properties of Flatback Airfoils," in 46th AIAA Aerospace Sciences Meeting and Exhibit, (Reno, NV), Jan. 2008.

[46] M. Barone and D. Berg, "Aerodynamic and Aeroacoustic Properties of a Flatback Airfoil: An Update," in 47th AIAA Aerospace Sciences Meeting Including The New Horizons Forum and Aerospace Exposition, (Orlando, FL), Jan. 2009.

[47] S. Guntur, A Detailed Study of the Rotational Augmentation and Dynamic Stall Phenomena for Wind Turbines. PhD thesis, Technical University of Denmark, Roskilde, DK, Dec. 2013.

[48] H. Snel, R. Houwink, and J. Bosschers, "Sectional Predictions of Lift Coefficients on Rotating Wind Turbine Blades in Stall," tech. rep., Netherlands Energy Research Foundation ECN, Amsterdam, NL, Dec. 1994.

[49] N. Troldberg, C. Bak, N. Sorensen, H. Madsen, P.-E. Rthor, F. Zahle, and S. Guntur, "Experimental and numerical investigation of 3D aerofoil characteristics on a MW wind turbine," tech. rep., Technical University of Denmark, Roskilde, DK.

[50] C. Lindenburg, "Modelling of Rotational Augmentation Based on Engineering Considerations and Measurements," in European Wind Energy Conference, (London, UK), Nov. 2004.

[51] C. Stone, S. Tebo, and E. Duque, "Computational Fluid Dynamics of Flatback Airfoils for Wind Turbine Applications," in 44th AIAA Aerospace Sciences Meeting and Exhibit, (Reno, NV), Jan. 2006.

[52] G. Papadakis, S. Voutsinas, G. Sieros, and T. Chaviaropoulos, "CFD aerodynamic analysis of non-conventional airfoil sections for very large rotor blades," in The Science of Making Torque from Wind, (Oldenburg, DE), Oct. 2012.

[53] J. Prospathopoulos, G. Papadakis, G. Sieros, S. Voutsinas, T. Chaviaropoulos, and K. Diakakis, "Assessment of the aerodynamic characteristics of thick airfoils 
in high Reynolds and moderate Ma numbers using CFD modeling," in The Science of Making Torque from Wind, (Copenhagen, DK), June 2014.

[54] C. Van Dam, E. Mayda, D. Chao, and D. Berg, "Computational design and analysis of flatback airfoil wind tunnel experiment," sandia report, Sandia National Laboratories, March 2008.

[55] J. Sanz, "CFD study of thick flatback arifoils using OpenFOAM," Master's thesis, Technical University of Denmark, Roskilde, DK, July 2011.

[56] E. Lynch, Advanced CFD Methods for Wind Turbine Analysis. PhD thesis, Georgia Institute of Technology, Atlanta, GA, May 2011.

[57] T. Kim, M. Jeon, S. Lee, and H. Shin, "Numerical simulation of flatback airfoil aerodynamic noise," Renewable Energy, vol. 65, May 2014.

[58] M. Blaylock, D. Maniaci, and B. Resor, "Numerical Simulations of Subscale Wind Turbine Rotor Inboard Airfoils at Low Reynolds Number," in AIAA SciTech 33rd Wind Energy Symposium, (Kissimmee, FL), Jan. 2015.

[59] A. Muñoz, B. Méndez, S. Gómez, A. Irisarri, and X. Munduate, New airfoils for high rotational speed wind turbines, ch. Chapter 3: Blade Root Airfoil Designs and Analysis of Aerodynamics- CENER. Innwind.EU, Sept. 2015.

[60] M. Maughmer and J. Coder, "Comparisons of Theoretical Methods for Predicting Airfoil Aerodynamic Characteristics ," tech. rep., U.S. Army Research, Development and Engineering Command, Port Matilda, PA, Aug. 2010.

[61] W. Shen, W. Zhu, A. Fischer, N. Garcia, J. Cheng, J. Chen, and J. Madsen, "Validation of the CQU-DTU-LN1 series of airfoils," in The Science of Making Torque from Wind, (Oldenburg, DE), Oct. 2012.

[62] W. Timmer and R. van Rooy, "Thick airfoils for HAWTS," Journal of Wind Engineering and Industrial Aerodynamics, vol. 39, 1992.

[63] Massachusetts Institute of Technology, "Area and Bending Inertia of Airfoil Sections ." Online: http:// ocw.mit.edu/courses/aeronautics-and-astronautics/ 16-01-unified-engineering-i-ii-iii-iv-fall-2005-spring-2006/ systems-labs-06/spl10b.pdf, Mar. 2016. 
[64] J. Paquette, J. van Dam, and S. Hughes, "Structural Testing of 9 m Carbon Fiber Wind Turbine Research Blades," in 45th AIAA Aerospace Sciences Meeting and Exhibit, Jan. 2007.

[65] D. Berry, "Blade System Design Studies Phase II: Final Project Report," tech. rep., Sandia National Laboratories, Albuquerque, NM, July 2008.

[66] C. van Dam, E. Mayda, D. Chao, K. Jackson, M. Zuteck, and D. Berry, "Innovative Structural and Aerodynamic Design Approaches for Large Wind Turbine Blades," tech. rep., Reno, NV, Jan. 2005.

[67] F. Hutcheson and T. Brooks, "Effects of Angle of Attack and Velocity on Trailing Edge Noise," tech. rep., NASA Langley Research Center, Hampton, VA, 2008 .

[68] T. Griffith and P. Richards, "The SNL100-03 Blade: Design Studies with Flatback Airfoils for the Sandia 100-meter Blade," tech. rep., Sandia National Laboratories, Albuquerque, NM, Sept. 2014.

[69] C. Bottasso, A. Croce, F. Grasso, and L. Sartori, "Free-Form Aerostructural Optimization of Rotor Blades," in European Wind Energy Association Conference, (Barcelona, ES), Mar. 2014. Poster.

[70] J. Tangler and D. Somers, "Status of the Special-Purpose Airfoil Families," tech. rep., Solar Energy Research Institute, Golden, CO, Oct. 1987.

[71] R. Eppler, Airfoil Design and Data. Berlin, DE: Springer, 1 ed., 1990.

[72] J. Tangler and D. Somers, "NREL Airfoil Families for HAWTs," tech. rep., National Renewable Energy Laboratory, Golden, CO, Jan. 1995.

[73] A. Bjorck, "Coordinates and Calculations For the FFA-W1-xxx, FFA-W2-xxx and FFA-W3-xxx Series of Airfoils for Horizontal Axis Wind Turbines," tech. rep., The Aeronautical Research Institue of Sweden, 1990.

[74] W. Timmer and R. van Rooij, "The Performance of New Wind Turbine Blade Tip and Root Airfoils up to High Angles-of-Attack," tech. rep., Delft University of Technology, Delft, NL.

[75] W. Timmer and R. van Rooij, "Summary of the Delft University Wind Turbine Dedicated Airfoils," American Institute of Aeronautics and Astronautics, 2003. 
[76] P. Fuglsang and C. Bak, "Development of the Riso Wind Turbine Airfoils," Wind Energy, vol. 7, 2004.

[77] C. Bak, P. Andersen, H. Madsen, and M. Gaunaa, "Design and Verification of Airfoils Resistant to Surface Contamination and Turbulence Intensity," in 26th AIAA Applied Aerodynamics Conference, (Honolulu, HI), Aug. 2008.

[78] P. Fuglsang, C. Bak, M. Gaunaa, and I. Antoniou, "Design and Verification of the Riso-B1 Airfoil for Wind Turbines," in 42nd AIAA Aerospace Sciences Meeting and Exhibit, (Reno, NV), American Institute of Aeronautics and Astronautics, Jan. 2004.

[79] X. Chen and R. Agarwal, "Optimization of Flatback Airfoils for Wind-Turbine Blades Using a Genetic Algorithm," Journal of Aircraft, vol. 49, pp. 622-629, Mar. 2012.

[80] M. Doosttalab and O. Frommann, "Effects of the Design Parameters on the Multidisciplinary Optimization of Flatback Airfoils for Large Wind Turbine Blades," (Fort Worth, TX), American Institute of Aeronautics and Astronautics, Sept. 2010.

[81] F. Zahle, C. Bak, N. Sorensen, T. Vronsky, and N. Gaudern, "Design of the LRP Airfoil Series Using 2D CFD," in The Science of Making Torque from Wind, (Copenhagen, DK), Journal of Physics: Conference Series, June 2014.

[82] C. Bak, N. Gaudern, F. Zahle, and T. Vronsky, "Airfoil design: Finding the balance between design lift and structural stiffness," in The Science of Making Torque from Wind, (Copenhagen, DK), Journal of Physics: Conference Series, June 2014.

[83] D. Simon, Evolutionary Optimization Algorithms. Hoboken, NJ: John Wiley \& Sons Inc., 2013.

[84] J. Nocedal and S. Wright, Numerical Optimization. New York, NY: Springer, 2nd ed., 2006.

[85] A. Jameson, "Gradient Based Optimization Methods," MAE Technical Report No. 2057, Princeton University, 1995.

[86] T. Holst and T. Pulliam, "Aerodynamic Shape Optimization Using a RealNumber-Encoded Genetic Algorithm," in 19th Applied Aerodynamics Conference, (Anaheim, CA), June 2001. 
[87] B. Chaparro, S. Thuiller, L. Menzes, P. Manach, and J. Fernandes, "Material parameters identification: Gradient-based, genetic and hybrid optimization algorithms," Computational Materials Science, Mar. 2008.

[88] D. Zingg, M. Nemec, and T. Pulliam, "A Comparative Evaluation of Genetic and Gradient-Based Algorithms Applied to Aerodynamic Optimization," REMN, vol. 17, Feb. 2008.

[89] The MathWorks, Natwick, MA, MATLAB Optimization Toolbox: User's Guide, 2016.

[90] K. Deb, "A Fast and Elitist Multiobjective Genetic Algorithm: NSGA-II," IEEE Transaction on Evolutionary Computation, vol. 6, Apr. 2002.

[91] MathWorks: MATLAB, Online: http://www.mathworks.com/help/gads/ genetic-algorithm-options, Genetic Algorithm Options, May 2016.

[92] A. Gardner and M. Selig, "Airfoil Design Using a Genetic Algorithm and an Inverse Method," in 41st Aerospace Sciences Meeting and Exhibit, (Reno, NV), Jan. 2003.

[93] D. Quagliarella and A. Della Cioppa, "Genetic Algorithms Applied to the Aerodynamic Design of Transonic Airfoils," Journal of Aircraft, vol. 32, Jan. 1995.

[94] "Patent US7660773 B1: System and method for the use of an adaptive mutation operator in genetic algorithms," 2005.

[95] F. Grasso and O. Ceyhan, "Usage of advanced thick airfoils for the outer part of very large offshore turbines," in The Science of Making Torque from Wind, (Copenhagen, DK), Journal of Physics: Conference Series, June 2014.

[96] D. Gries and F. Schneider, eds., The Computer Graphics Manual, vol. 1, ch. Bezier Approximation. London, UK: Springer, 2011.

[97] I. Danaila, P. Joly, S. Kaber, and M. Postel, An Introduction to Scientific Computing, ch. Geometrical Design: Bezier Curves and Surfaces. New York, NY: Springer, 2007.

[98] R. de Oliviera, "XFOIL-MATLAB Interface." Online: https://www . mathworks.com/matlabcentral/fileexchange/ 30478-xfoil-matlab-interface, Feb. 2011. 
[99] G. Ramanujam, H. Ozdemir, and H. Hoeijmakers, "Improving Airfoil Drag Prediction," Journal of Aircraft, June 2016.

[100] Leishman, J. and Beddoes, T., "A Generalized Model for Airfoil Unsteady Aerodynamic Behavior and Dynamic Stall Using the Indicial Method," in 42nd Annual Forum of the American Helicopter Society, (Washington, DC), June 1986.

[101] F. White, Viscous Fluid Flow. New York, NY: McGraw-Hill, 3 ed., 2006.

[102] J. van Ingen, "The eN method for transition prediction. Historical review of work at TU Delft," in 38th Fluid Dynamics Conference and Exhibit, (Seattle,WA), AIAA, June 2008.

[103] L. Mack, "Aerodynamic Analysis Requiring Advanced Computers," tech. rep., NASA, Washington, DC, Mar. 1975.

[104] M. Sessarego, "A Hybrid Multi-Objective Evolutionary Algorithm for WindTurbine Blade Optimization," Master's thesis, University of Calgary, Calgary, AB, Apr. 2013.

[105] P. Dijkstra, "Rotor noise and aero-acoustic optimization of wind turbine airfoils," Master's thesis, Delft University of Technology, Delft, ND, July 2015.

[106] G. de Oliveira, "Wind Turbine Airfoils with Boundary Layer Suction, a Novel Design Approach," Master's thesis, Delft University of Technology, Delft, ND, July 2011.

[107] P. Fuglsang, I. Antoniou, K. Dahl, and H. Madsen, "Wind Tunnel Tests of the FFA-W-241, FFA-W3-301 and NACA63-430 Airfoils," tech. rep., Riso National Laboratory, Roskilde, DK, 121998.

[108] R. Ehrmann, E. White, C. Maniaci, R. Chow, C. Langel, and C. van Dam, "Realistic Leading-Edge Roughness Effects on Airfoil Performance," in 31st AIAA Applied Aerodynamics Conference, AIAA, June 2013.

[109] A. Sareen, C. Sapre, and M. Selig, "Effects of Leading Edge Erosion on Wind Turbine Blade Performance," in Wind Energy, no. 17, pp. 1532-1542, 2014.

[110] D. Somers, "Effects of Airfoil Thickness and Maximum Lift Coefficient on Roughness Sensitivity," tech. rep., National Renewable Energy Laboratory, Golden, CO, Jan. 2005. 
[111] R. van Rooij and W. Timmer, "Roughness Sensitivity Considerations for Thick Rotor Blade Airfoils," Journal of Solar Energy Engineering, vol. 125, Nov. 2003.

[112] K. Schweigler, "Aerodynamic Analysis of the NREL 5-MW Wind Turbine using Vortex Panel Method," Master's thesis, Chalmers University of Technology, Gothenbug, SE, 2012.

[113] C. Gutierrez, "Aerodynamic and aeroelastic design of low wind speed wind turbine blades," Master's thesis, Technical University of Denmark, Lyngby, DK, 2014.

[114] J. Cimbala, "Taguchi Orthogonal Arrays." Online: http://www.mne.psu.edu/ cimbala/me345/Lectures/Taguchi_orthogonal_arrays.pdf, Sept. 2014.

[115] R. van Rooij, "Modification of the boundary layer calculation in RFOIL for improved airfoil stall prediction," tech. rep., Wind Energy- Delft University of Technology, Delft, NE, Sept. 1996.

[116] F. Bertagnolio, N. Sorensen, J. Johansen, and P. Fuglsang, "Wind Turbine Airfoil Catalogue," tech. rep., Riso National Laboratory, Roskilde, DK, Aug. 2001.

[117] E. El-Ramly and W. Rainbird, "Flow Survey of the Vortex Wake behind Wings," Journal of Aircraft, vol. 14, pp. 1102-1108, June 1977.

[118] S. Roberts, "Boundary Layer Transition in Attached and Separated Flows at Low Reynolds Numbers," MASc Thesis, Carleton University, Ottawa, ON, May 2005.

[119] D. Brassard, "A 2D Transverse Vortex Wind Tunnel for PIV Investigation of Airfoil Vortex Interaction," Master's thesis, Carleton University, Ottawa, ON, Aug. 2005.

[120] W. Burwash, "Particle Image Velocimetry Measurements of an Airfoil-Vortex Interaction Event in a Two-Dimensional Wind Tunnel," Master's thesis, Carleton University, Ottawa, ON, Aug. 2007.

[121] ATI Industrial Automation, "F/T Sensor: Delta." Online: http://www . ati-ia.com/products/ft/ft_models.aspx?id=Delta, July 2016.

[122] A. Pope and J. Harper, Low-Speed Wind Tunnel Testing. John Wiley \& Sons Inc., 1966. 
[123] R. Ramsay, M. Hoffman, and G. Gregorek, "Effects of Grit Roughness and Pitch Oscillations on the S809 Airfoil," tech. rep., National Renewable Energy Laboratory, Golden, CO, Dec. 1995.

[124] Environment Canada, "Ottawa (Kanata-Orleans), ON." Online: https:// weather.gc.ca/city/pages/on-118_metric_e.html, 2016.

[125] Lee Valley, "Digital protractor." Online: http://www.leevalley.com/en/ Wood/page. aspx?p=64278\&cat=1, 43513, July 2016.

[126] Huntsman, "Renshape 5045." Online: https://www.freemansupply.com/ datasheets/RenShapePolyToolingBoards/5045.pdf, July 2016.

[127] Top Flite, "Econokote." Online: http://www.monokote.com/econokote.html, July 2016.

[128] Coverite, "21st Century Sealing Iron." Online: http://www.coverite.com/ accys/covr2700.html, July 2016.

[129] R. Ramsay, J. Janiszewska, and G. Gregorek, "Wind Tunnel Testing of Three S809 Aileron Configurations for use on Horizontal Axis Wind Turbines," tech. rep., The Ohio State University, Colombus, OH, July 1996.

[130] A. Thom, "Blockage Corrections in a High Speed Wind Tunnel," R\&M no. $2033,1943$.

[131] E. Maskell, "A Theory of the Blockage Effects on Bluff Bodies and Stalled Wings in a Closed Wind Tunnel," R\&M, no. 3400, 195.

[132] M. Hand, D. Simms, L. Fingersh, D. Jager, J. Cotrell, S. Schreck, and S. Larwood, "Unsteady Aerodynamics Experiment Phase VI: Wind Tunnel Test Configurations and Available Data Campaigns," tech. rep., National Renewable Energy Laboratory, Golden, CO, Dec. 2001.

[133] R. Sheldahl and P. Klimas, "Aerodynamic Characteristics of Seven Symmetrical Airfoil Sections Through 180-Degree Angle of Attack for Use in Aerodynamic Analysis of Vertical Axis Wind Turbines," tech. rep., Sandia National Laboratories, Albuquerque, NM, Mar. 1981.

[134] Carleton University, "MECH5008 Experimental Methods in Fluid Mechanics: Course Notes." Sept. 2015. 
[135] Pham, ed., Handbook of Engineering Statistics. London, UK: Springer, 2006.

[136] Michigan State University, "Error propagation." Online: http: //lectureonline.cl.msu.edu/ mmp/labs/error/e2.htm, Aug. 2016.

[137] M. Manolesos, G. Papadakis, and S. Voutsinas, "Study of Drag Reduction Devices on a Flatback Airfoil," in AIAA SciTech Forum: 34th Wind Energy Symposium, (San Diego, CA), Jan. 2016.

[138] M. Manolesos, G. Papadakis, and S. Voutsinas, "An experimental and numerical investigation on the formation of stall-cells on airfoils," in The Science of Making Torque from Wind, (Oldenburg, DE), Oct. 2012.

[139] A. Cooperman, A. McLennan, R. Chow, J. Baker, and van Dam, C.P., "Aerodynamic Performance of Thick Blunt Trailing Edge Airfoils," in 28th AIAA Applied Aerodynamics Conference, (Chicago, IL), American Institute of Aeronautics and Astronautics, June 2010.

[140] J. Blasques, BECAS: A cross section analysis tool for anisotropic and inhomogeneous beam sections of arbitrary geometry. Department of Wind EnergyTechnical University of Denmark, Oct. 2015. 